University of New Hampshire

University of New Hampshire Scholars' Repository

\title{
Instrument description and performance of the Imaging Gamma- Ray Telescope COMPTEL aboard the Compton Gamma-Ray Observatory
}

\author{
V. Schonfelder \\ Max-Planck-Institut für extraterrestriche Physik \\ H. Aarts \\ ESTEC \\ K Bennett \\ ESTEC \\ $\mathrm{H}$ deBoer \\ SRON \\ Follow this and additional works at: https://scholars.unh.edu/ssc \\ J. Clear

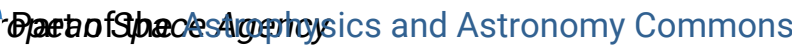

\section{Comments}

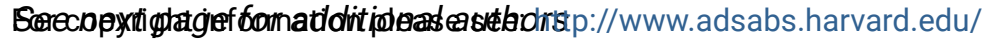

\section{Recommended Citation}

V. Schoenfelder, H. Aarts, K. Bennett, H. de Boer, J. Clear, W. Collmar, A. Connors, A. Deerenberg, R. Diehl, A. von Dordrecht, J. W. den Herder, W. Hermsen, M. Kippen, L. Kuiper, G. Lichti, J. Lockwood, J. Macri, M. McConnell, D. Morris, R. Much, J. Ryan, G. Simpson, M. Snelling, G. Stacy, H. Steinle, A. Strong, B. N. Swanenburg, B. Taylor, C. de Vries, and C. Winkler, 'Instrument description and performance of the Imaging Gamma-Ray Telescope COMPTEL aboard the Compton Gamma-Ray Observatory', The Astrophysical Journal Supplement Series, vol. 86, p. 657, May 1993.

This Article is brought to you for free and open access by the Institute for the Study of Earth, Oceans, and Space (EOS) at University of New Hampshire Scholars' Repository. It has been accepted for inclusion in Space Science Center by an authorized administrator of University of New Hampshire Scholars' Repository. For more information, please contact Scholarly.Communication@unh.edu. 


\section{Authors}

V. Schonfelder, H. Aarts, K Bennett, H deBoer, J. Clear, W Collmar, A Connors, A. Deerenberg, R Diehl, A. von Dordrecht, J W. den Herder, W Hermsen, R M. Kippen, L Kuiper, G G. Lichti, J Lockwood, John R. Macri, Mark L. McConnell, D Morris, R Much, James M. Ryan, G. Simpson, M. Snelling, G Stacy, H Steinle, A W.

Strong, B Swanenburg, B G. Taylor, C de Vries, and C Winkler 
THE ASTROPHYSICAL JOURNAL SUPPLEMENT SERIES, 86:657-692, 1993 June

(c) 1993. The American Astronomical Society. All rights reserved. Printed in U.S.A.

V. Schönfelder, ${ }^{1}$ H. Aarts,${ }^{2}$ K. Bennett,${ }^{3}$ H. de Boer,${ }^{2}$ J. Clear,${ }^{3}$ W. Collmar, ${ }^{1}$ A. Connors,${ }^{4}$ A. Deerenberg, ${ }^{2}$ R. Diehl, ${ }^{1}$ A. von Dordrecht ${ }^{3}$ J. W. DEN Herder, ${ }^{2}$ W. HermSEn, ${ }^{2}$ M. KiPPEN, ${ }^{4}$ L. KuIPER, ${ }^{2}$ G. LiCHTI,${ }^{1}$ J. LoCKWOOD, ${ }^{4}$ J. MaCri, ${ }^{4}$ M. McConnell, ${ }^{4}$ D. Morris,${ }^{4}$ R. Much, ${ }^{1}$ J. Ryan, ${ }^{4}$ G. Simpson, ${ }^{4}$ M. SNElling,${ }^{3}$ G. Stacy, ${ }^{4}$ H. Steinle,${ }^{1}$ A. Strong, ${ }^{1}$ B. N. Swanenburg, ${ }^{2}$ B. TAYlor,${ }^{3}$ C. DE VRIES, ${ }^{2}$ AND C. WinKLeR ${ }^{3}$

Received 1992 July 21; accepted 1992 October 28

1. Introduction

2. Principle of Measurement

3. Instrument Description

3.1. $D_{1}$ Detector Assembly

3.2. $\mathrm{D}_{2}$ Detector Assembly

3.3. Veto Domes

3.4. Calibration Units

3.5. Electronic Subsystem

3.6. Single-Detector Burst-Mode Description

3.7. Other Modes of Operation

3.8. Mechanical Structure and Thermal Control

4. Determination of Instrument Characteristics

4.1. Telescope Mode Calibration

4.2. Calibration of Individual Detector Modules

4.3. Monte Carlo Simulations

5. Performance of Main Detector Elements

5.1. Energy Loss Assignment and Gain Corrections

5.2. $\mathrm{D}_{1}$ Module Performance

5.3. $\mathrm{D}_{2}$ Module Performance

\section{CONTENTS}

Page Fiche

$658 \quad 23-\mathrm{A} 6$

$659 \quad 23-A 7$

661 23-A9

$661 \quad 23-A 9$

$662 \quad 23-\mathrm{A} 10$

663 23-A11

663 23-A11

665 23-A 13

666 23-A14

666 23-A14

$667 \quad 23-\mathrm{B} 1$

$667 \quad 23-\mathrm{B} 1$

$668 \quad 23-B 2$

$669 \quad 23-\mathrm{B} 3$

669 23-B3

$670 \quad 23-\mathrm{B} 4$

$670 \quad 23-B 4$

672 23-B6

$674 \quad 23-B 8$
5.4. Time-of-Flight Measurements

5.5. Veto-Dome Performance

6. Telescope Performance

6.1. The Imaging Gamma-Ray Telescope

6.2. Response to Gamma-Ray Bursts

6.3. The COMPTEL Neutron Response

7. Demonstration of COMPTEL Imaging Capabilities

7.1. Maximum-Entropy Imaging of COMPTEL Data

7.2. Maximum-Likelihood Method for COMPTEL Source Recognition and Parameter Estimation

8. COMPTEL Sensitivity

8.1. In-Orbit Background

8.2. Point-Source Sensitivity

8.3. Sensitivity to Burst Detection

8.4. Sensitivity to Polarization Measurements

8.5. Sensitivity to Solar Neutrons

9. Outlook
Page Fiche

674 23-B8

$675 \quad 23-\mathrm{B} 9$

$675 \quad 23-\mathrm{B} 9$

$676 \quad 23-\mathrm{B} 10$

$680 \quad 23-\mathrm{B} 14$

$681 \quad 23-\mathrm{C} 1$

685 23-C5

685 23-C5

$686 \quad 23-\mathrm{C} 6$

$688 \quad 23-\mathrm{C} 8$

$688 \quad 23-\mathrm{C} 8$

$688 \quad 23-\mathrm{C} 8$

689 23-C 9

$690 \quad 23-\mathrm{C} 10$

691 23-C11

691 23-C11

\section{ABSTRACT}

The imaging Compton telescope COMPTEL is one of the four instruments on board the Compton GammaRay Observatory (GRO), which was launched on 1991 April 5 by the space shuttle Atlantis into an Earth orbit of $450 \mathrm{~km}$ altitude. COMPTEL is exploring the $1-30 \mathrm{MeV}$ energy range with an angular resolution $(1 \sigma)$ between $1^{\circ}$ and $2^{\circ}$ within a large field of view of about 1 steradian. Its energy resolution $(8.8 \% \mathrm{FWHM}$ at $1.27 \mathrm{MeV})$ makes it a powerful gamma-ray line spectrometer. Its effective area (for on-axis incidence) varies between 10 and $50 \mathrm{~cm}^{2}$ depending on energy and event selections. Within a 14 day observation period COMPTEL is able to detect sources which are about 20 times weaker than the Crab. The measurement principle of COMPTEL also allows the measurements of solar neutrons. COMPTEL's neutron sensitivity above $15 \mathrm{MeV}$ is 5 neutrons cm${ }^{-2}$ for a 30 minute observation time. In a so-called single-detector mode, COMPTEL uses two of its $\mathrm{D}_{2}$ modules to study the temporal spectral evolution of gamma-ray bursts or other transients. In its telescope mode COMPTEL is performing the first complete sky survey at $\mathrm{MeV}$ energies during the first 18 months of the mission. Targets of special interest are Galactic gamma-ray sources ( such as radio pulsars, X-ray binaries, the Galactic center, the unidentified $\operatorname{COS} B$ sources, supernova remnants, and molecular clouds), external galaxies (especially the nuclei of active galaxies), gamma-ray line sources (e.g., the distribution of the $1.8 \mathrm{MeV}$ line emissivity throughout the Galaxy), the diffuse gamma-ray emission from interstellar space, the cosmic gamma-ray background, cosmic gamma-ray bursts, and gamma-ray and neutron emission during solar flares. In later phases of the mission, the objects discovered in the survey will be studied in more detail. This paper provides a detailed description of the instrument: its principle of measurement, design, and performance. The telescope characteristics were derived from calibration measurements and simulations. First flight data are being used to demonstrate its in-flight performance and to derive the achieved sensitivity of the telescope.

Subject headings: artificial satellites, space probes — gamma rays: observations — instrumentation: detectors - telescopes

\footnotetext{
${ }^{1}$ Max-Planck-Institut für Extraterrestrische Physik, D-8046 Garching bei München, Germany.

${ }^{2}$ Laboratory for Space Research Leiden, NL-2333 AL Leiden, The Netherlands.

${ }^{3}$ Space Science Department, European Space Agency, NL-2201 AZ Noordwijk, The Netherlands.

${ }^{4}$ Space Science Center, University of New Hampshire, Durham, NH 03824.
} 


\section{INTRODUCTION}

The exploration of the sky in the low-energy gamma-ray range between 1 and $30 \mathrm{MeV}$ lags far behind that in the X-ray band and at higher gamma-ray energies. The imaging Compton telescope COMPTEL aboard the Compton Gamma-Ray Observatory $(G R O)$ will for the first time bridge that gap. The few results that do exist at these energies have demonstrated that the scientific return of $\mathrm{MeV}$ gamma-ray astronomy is very high. Quite different physical processes dominate the emission at X-ray and low and high gamma-ray energies. Important production processes in the COMPTEL energy range are nuclear de-excitation, radioactive decay, positron annihilation, bremsstrahlung, Compton scattering, synchrotron emission, and thermal emission ( $k T$ greater than several hundred $\mathrm{keV}$ ). A variety of astronomical phenomena can be studied through the window of low-energy gamma-ray astronomy. Promising objects in the Milky Way comprise the final stages of stellar evolution: neutron stars, black holes, novae, and supernova outbursts. Many of the gamma-ray burst sources have been shown to be strong $\mathrm{MeV}$ emitters, and also the Sun is an interesting source during solar flares. The interstellar medium is an extended source of gamma-ray emission. Here we see either radiation produced in cosmic-ray interactions or radiation from radioactive material that was ejected into interstellar space. In the extragalactic sky the nuclei of active galaxies and the cosmic gamma-ray background are the most interesting targets. The bulk of the power of many active galaxies seems to be at $\mathrm{MeV}$ energies, and also the cosmic background radiation shows an enhanced emission feature at these energies.

Since $G R O$ offers the first opportunity for an in-depth investigation of the sky at low gamma-ray energies, an instrument with a wide field of view and a good angular resolution within the field is needed to be capable of observing a variety of unexpected phenomena. COMPTEL provides these properties. Its field of view is $\approx 1 \mathrm{sr}$, and its angular resolution is a few degrees. In addition, COMPTEL has good energy resolution ranging from $5 \%$ to $10 \%$ FWHM, and is able to detect polarized emission from unusually strong sources. With these properties COMPTEL is well suited for surveying the entire sky, and especially for mapping the Milky Way in the light of continuum and line emission. The principle of measurement of COMPTEL facilitates measurement of neutrons in addition to gamma rays. In a special mode of operation COMPTEL can therefore also be used to observe solar neutron emission during large flares.

The development of the Compton telescope technique began at the Max-Planck-Institut in 1971 (Schönfelder, Hirner, \& Schneider 1973). Similar developments have been performed at the University of California, Riverside (Herzo et al. 1975), and at the University of New Hampshire (Lockwood et al. 1979, 1981). The performance of the Compton telescope technique was investigated in many balloon flights. COMPTEL's design is essentially based on the configuration of the MPI balloon-borne Compton telescope (Schönfelder, Graser, \& Diehl 1982), with improvements in many areas. COMPTEL is the first Compton telescope flown on a satellite.

COMPTEL was proposed to NASA in 1978 in response to the Announcement of Opportunity for instruments on board the Gamma-Ray Observatory (GRO) by an international col- laboration consisting of the Max-Planck-Institut für Extraterrestrische Physik in Garching, the Laboratory for Space Research in Leiden, the University of New Hampshire in Durham, and the Space Science Department of the European Space Agency in Noordwijk. In 1981, at the end of a 3 year definition phase, COMPTEL was finally selected by NASA as one of the four $G R O$ instruments.

A schematic view of $G R O$ is shown in Figure 1. COMPTEL occupies the middle position of the spacecraft platform; the Energetic Gamma-Ray Experiment Telescope (EGRET) (Fichtel et al. 1983) is to the right, and the Oriented Scintillation Spectroscopy Experiment (OSSE) (Kurfess et al. 1983) to the left. The Burst and Transient Source Experiment (BATSE) (Fishman et al. 1985) consists of eight single detectors, two at each corner of the spacecraft.

$G R O$ is a free-flying satellite which was launched by the shuttle Atlantis on 1991 April 5. GRO is three-axis-stabilized with a pointing accuracy of \pm 0.5 , the pointing direction being known at any time to an accuracy of $2^{\prime}$. Absolute COMPTEL event time is accurate to $0.125 \mathrm{~ms}$, the spacecraft clock being always within $10 \mu$ s of Universal Time. GRO has a circular orbit of $450 \mathrm{~km}$ and 28.5 inclination. This orbit guarantees a mission lifetime of at least 2 years and at the same time provides a low background environment. On the other side, about two-thirds of the observation time of COMPTEL is lost due to occultation of the field of view by the Earth. The nominal observation time of $G R O$ is 2 weeks per viewing direction. During the first 15 months of the mission, a complete sky survey is being made. During later phases of the mission detailed observations of selected objects are foreseen.

The present paper describes the COMPTEL instrument and its properties. The determination of the properties is based on calibration measurements and Monte Carlo simulations. The calibration was performed with radioactive sources and nuclear reaction gamma rays and neutrons, produced by an accelerator. From these calibration data, the angular and energy response and the effective detection area have been determined and the imaging properties of COMPTEL demonstrated. The sensitivity of COMPTEL to celestial gamma-ray sources was estimated using flight data.

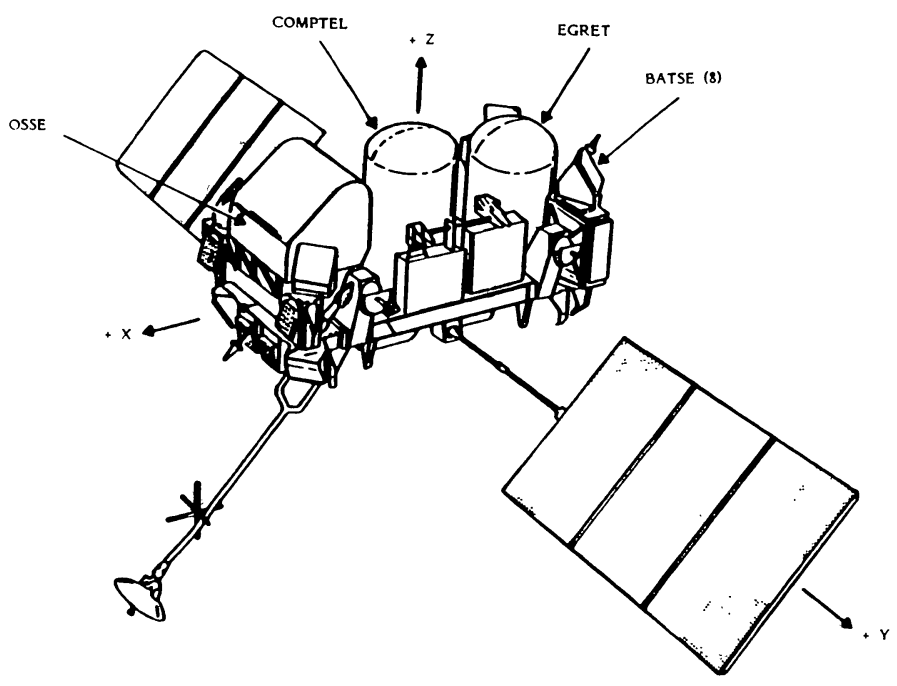

FIG. 1.-Schematic view of $G R O$ 


\section{PRINCIPLE OF MEASUREMENT}

The principle of measurement is illustrated in Figure 2. COMPTEL consists of two detector arrays, an upper one of low- $Z$ material (liquid scintillator NE 213A) and a lower one of high- $Z$ material [ $\mathrm{NaI}(\mathrm{Tl})$ scintillator]. In the upper detector $\left(D_{1}\right)$ a gamma ray is Compton-scattered, and then the scattered gamma ray makes a second interaction in the lower detector $\left(D_{2}\right)$. The sequence is confirmed by a time-of-flight measurement. The locations and energy losses of both interactions are measured. For completely absorbed events the arrival direction of the gamma ray is known to lie on the edge of a cone, whose axis is the direction of the scattered gamma ray; $\bar{\varphi}$ is the angle between the arrival direction and the scatter direction (see Fig. 2), where

$$
\cos \bar{\varphi}=1-\frac{m_{0} c^{2}}{E_{2}}+\frac{m_{0} c^{2}}{E_{1}+E_{2}} .
$$

$E_{1}$ and $E_{2}$ are the measured energy losses in $\mathrm{D}_{1}$ and $\mathrm{D}_{2}$, respectively; $m_{0} c^{2}$ is the electron rest energy. A celestial source therefore can be located from the circles of different gamma rays from this source. Incompletely absorbed events produce circles

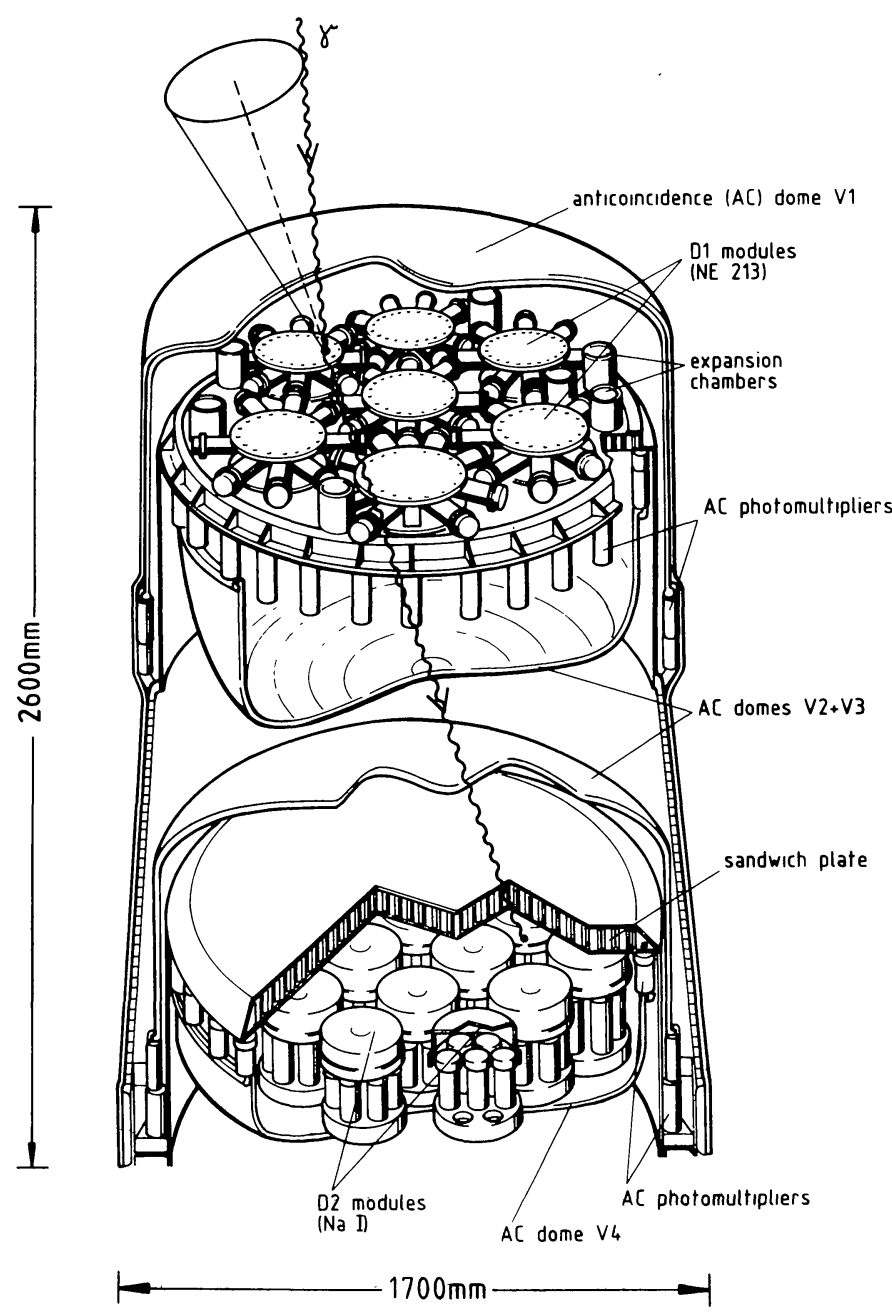

FIG. 2.-Schematic view of COMPTEL which do not cross the source position. The angular resolution of the telescope depends on the accuracy to which $\bar{\varphi}$ and the direction of the scattered gamma ray are determined. In some ways COMPTEL is similar to an optical camera: the first detector, which can be compared with the camera's lens, directs the light into the second detector, comparable to the film, in which the scattered photon is absorbed. Although the photons are not focused as in the case of the optical camera, COMPTEL is able to reconstruct sky images over a wide field of view with a resolution of about $1^{\circ}$.

The two detectors are separated by a distance of $1.5 \mathrm{~m}$. Each detector is entirely surrounded by a thin anticoincidence shield of plastic scintillator, which is used to reject charged particles. Off to the sides between the two detectors are two small plastic scintillation detectors containing weak ${ }^{60} \mathrm{Co}$ sources; these are used as electronically gated gamma-ray calibration sources.

A gamma ray is electronically identified by a delayed coincidence between the upper and the lower detector, combined with the absence of a veto signal from all charged particle shields and from the calibration units. The quantities measured for each event are as follows:

1. The energy loss $E_{1}$ of the Compton electron in the upper detector.

2. The location of the interaction in the upper detector.

3. The pulse shape of the scintillation pulse in the upper detector.

4. The energy loss $E_{2}$ in the lower detector.

5. The location of the interaction in the lower detector.

6. The time of flight of the scattered gamma ray from the upper to the lower detector.

7. The time of the event.

The locations of the interactions are derived from the relative pulse heights of all photomultipliers viewing one detector module. The pulse-shape measurements and the time-of-flight measurements are performed in order to reject background events.

Although the effective detection area of a Compton telescope is small-owing to the requirement of subsequent interactions in two different detectors-a high sensitivity is still achieved because gamma rays from a celestial source are distinguished from background events by the multiparameter signature of each event. Background rejection by means of the veto, the time of flight, the pulse-shape techniques and the kinematics of the Compton collisions lead to a favorable signal-tonoise ratio. The relationship between $E_{\gamma}, E_{1}, E_{2}$, and $\bar{\varphi}$ (see eq. [1]) is illustrated in Figure 3.

Two important conclusions can be derived from this diagram. First, the hardware energy thresholds of $E_{1}$ and $E_{2}$ have a significant effect on the field of view of the telescope and, hence, on the total event rate which is to be processed. The $E_{1}$ threshold defines the lower limit, and the $E_{2}$ threshold the upper limit of accepted scatter angles:

$$
\bar{\varphi}_{\min }\left(E_{1} \text { threshold }<\bar{\varphi}<\bar{\varphi}_{\max }\left(E_{2} \text { threshold }\right)\right.
$$

At a $D_{1}$ threshold of $50 \mathrm{keV}$ the minimum scatter angles range from $19^{\circ}$ at $E_{\gamma}=700 \mathrm{keV}$ to $1^{\circ}$ at $E_{\gamma}=30 \mathrm{MeV}$. At a $\mathrm{D}_{2}$ threshold of $500 \mathrm{keV}$ the maximum accepted scatter angles range from $45^{\circ}$ at $700 \mathrm{keV}$ to $90^{\circ}$ at $30 \mathrm{MeV}$. A lower value of 


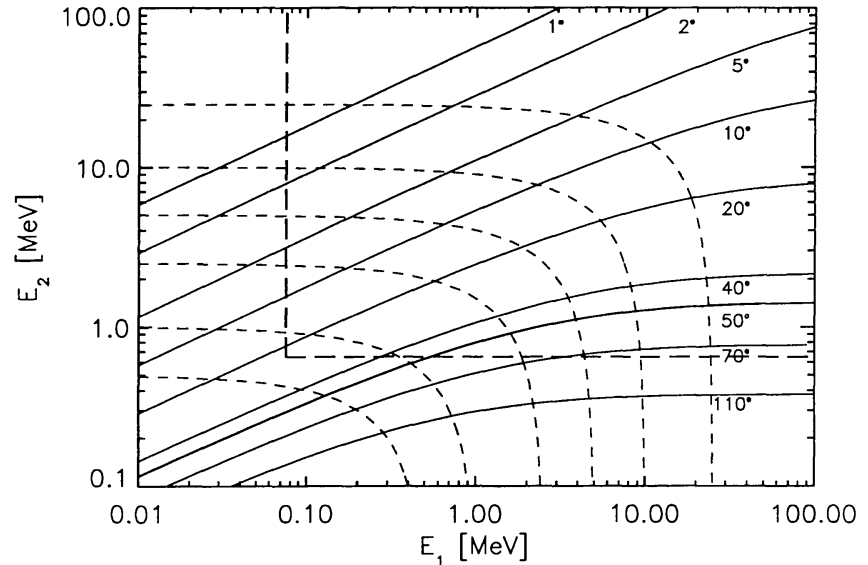

FIG. 3.-Relation between $E_{\gamma}$ (dashed lines), $E_{1}, E_{2}$, and $\bar{\varphi}$. The impact of the energy thresholds used in the flight data analysis is indicated by the lines.

the $E_{2}$ threshold leads to a widening of the field of view, and hence to a dramatic increase of the COMPTEL event rate.

Second, the field of view of the telescope can be further limited by introducing $\bar{\varphi}$ restrictions for events which are to be accepted. Events with small scatter angles lead to a better signal-to-noise ratio than events with high values. However, any restriction in $\bar{\varphi}$ implies also a loss in detection efficiency. In order to illustrate the impact of $\bar{\varphi}$ restrictions, the calibration data have been analyzed for the two cases of no restriction and the following "extreme" restriction:

$$
\begin{array}{ll}
\bar{\varphi}<30^{\circ} & \text { for } E_{1}+E_{2}<2.6 \mathrm{MeV}, \\
E_{1} \leq 0.4\left(E_{1}+E_{2}\right) & \text { for } 2.6 \mathrm{MeV}<E_{1}+E_{2}<10 \mathrm{MeV}, \\
\bar{\varphi}<15^{\circ} & \text { for } E_{1}+E_{2} \geq 10 \mathrm{MeV} .
\end{array}
$$

The effect of any other $\bar{\varphi}$ restriction or any restriction of the $E_{1} / E_{2}$ ratio on the field of view can easily be derived from Figure 3.

The COMPTEL point-spread function can be described in the simplest way in a three-dimensional data space defined by two orthogonal coordinates $(\chi, \psi)$ describing the direction of the scattered gamma ray and the Compton scatter angle $\bar{\varphi}$. Each recorded gamma-ray event is represented by one single point in this data space. In the idealized case, in which the scattered gamma ray is totally absorbed in the lower detector, the pattern of data points from a gamma-ray point source with the coordinates $\left(\chi_{0}, \psi_{0}\right)$ lies on a cone mantle in $(\chi, \psi, \bar{\varphi})$ space, where the cone apex is at $\left(\chi_{0}, \psi_{0}\right)$ and the cone semiangle is $45^{\circ}$ (see Fig. 4).

The response density along the cone follows the Klein-Nishina cross section. This idealized "cone mantle" response is blurred by measurement inadequacies in the scintillation detection, especially by incompletely absorbed events in $D_{2}$, which fill the interior of the cone to a certain extent. If the gamma-ray emission of the point source is linearly polarized, the scattered gamma ray will be found preferentially in the plane perpendicular to the polarization vector. The event den- sity around the cone again follows the Klein-Nishina cross section for polarized radiation (Heitler 1954).

The double scattering process that is used in COMPTEL to detect gamma rays can also be used to measure neutrons in energy and angle (Pinkau 1966; White 1968). The ideal type of neutron interaction in COMPTEL occurs when the incoming neutron elastically scatters off a hydrogen nucleus in the $D_{1}$ detector and subsequently interacts in a $\mathrm{D}_{2}$ module (see Fig. 5 ). The energy of the incident neutron is computed by summing the proton recoil energy $E_{p}$ in the $\mathrm{D}_{1}$ detector with the energy of the scattered neutron, $E_{n}^{\prime}$, which is deduced from the time-of-flight measurement from the $\mathrm{D}_{1}$ to the $\mathrm{D}_{2}$ detector. The scatter angle for relativistic neutrons can be computed from the formula

$$
\tan ^{2} \Theta_{n}=\frac{E_{p}}{E_{n}^{\prime}} \frac{1}{1+E_{n} / 2 M_{n} c^{2}} \quad \text { with } E_{n}=E_{p}+E_{n}^{\prime}
$$

( $\Theta_{n}$ is the neutron scatter angle, and $M_{n} c^{2}$ is the rest energy of the neutron). Again, the incident direction of the infalling neutron is determined to lie on a cone mantle of half-opening angle $\Theta_{n}$ around the direction of the scattered neutron. Other inelastic reactions with carbon also occur in the liquid scintillation, especially at energies greater than $50 \mathrm{MeV}$. These interactions are difficult to interpret, because gamma rays, protons, deuterons, or $\alpha$-particles can be produced. In the case of a solar

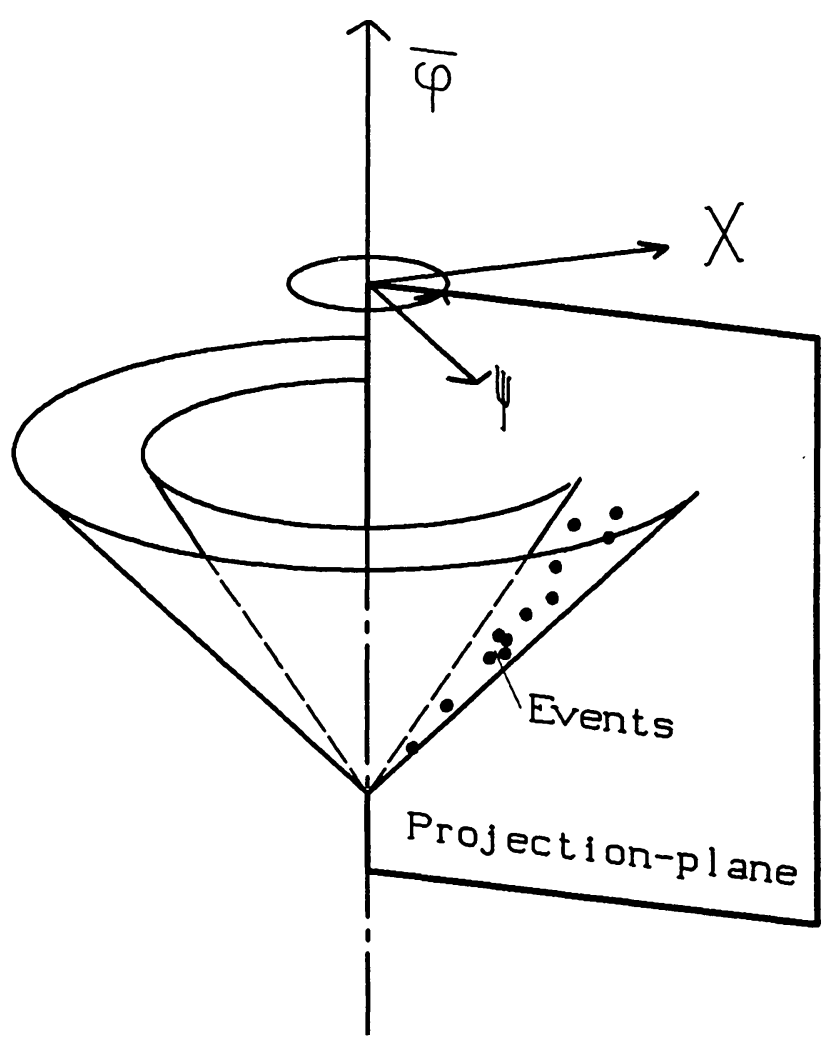

FIG. 4.-Illustration of the COMPTEL response of a celestial point source in the three-dimensional data space $(\chi, \psi, \bar{\varphi})$. The data lie on a cone, the apex of which is at the position of the celestial source $\left(\chi_{0}, \psi_{0}\right)$. The cone semiangle is $45^{\circ}$. In practice, the cone mantle is blurred because of measurement inaccuracies. 


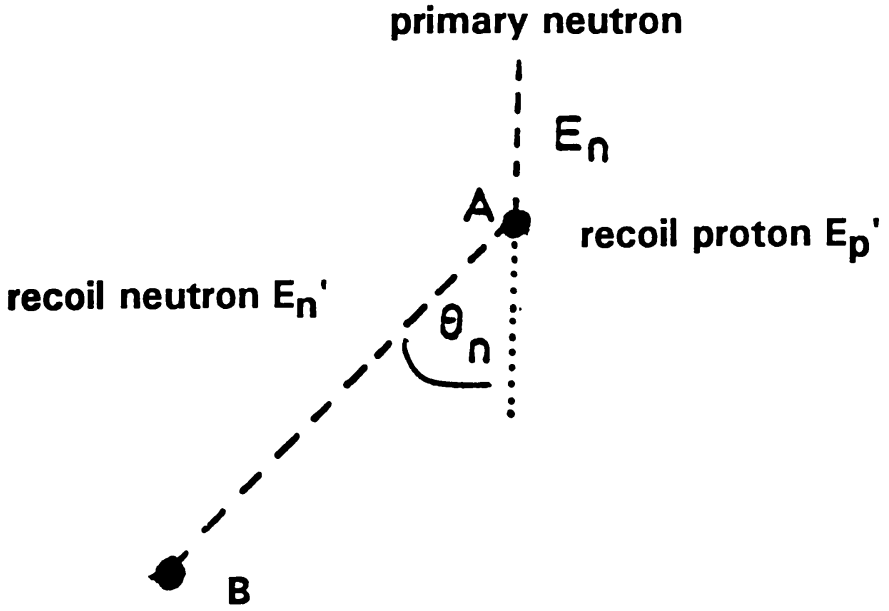

Fig. 5.- Illustration of the principle of neutron measurement in COMPTEL. The collision at point $A$ occurs in $D_{1}$; the one at point $B$ is in $\mathrm{D}_{2}$.

flare, these events can be used to supplement the information obtained from the elastic scatters.

In addition to the normal double-scattering mode of measuring gamma rays and neutrons, two of the $\mathrm{D}_{2}$ modules of the telescope are used to measure the energy spectra of cosmic gamma-ray bursts and solar flares. The trigger for such an event is provided by BATSE. In case of a solar flare COMPTEL receives a set of commands initiated by the onboard computer serving the BATSE solar trigger. COMPTEL then goes into an alternate event-selection mode, which allows the measurement of solar neutrons in addition to gamma rays.

Neutron events are identified by their pulse shape and timeof-flight signature. In the solar neutron mode, events with a neutron signature are not rejected but are recorded and transmitted with secondary priority.

\section{INSTRUMENT DESCRIPTION}

\section{1. $D_{1}$ Detector Assembly}

The COMPTEL upper detector assembly $\left(D_{1}\right)$ consists of seven cells, each of which is $28 \mathrm{~cm}$ in diameter and $8.5 \mathrm{~cm}$ deep, filled with the liquid scintillator NE 213A. These cells are mounted on a circular aluminum plate $1.45 \mathrm{~m}$ in diameter. Each cell is viewed by eight EMI 9755 NA photomultiplier tubes (PMTs) which look into the cells through fused silica windows. The PMTs are located uniformly around the circumference of the cell and are enclosed in an aluminum housing, which also holds the high-voltage bleeder string. The high-voltage power supply boxes (HVPSs), high-voltage junction boxes (HVJBs), and front-end electronics boxes (FEEs) are mounted on the underside of the platform out of the gammaray path between the $D_{1}$ and $D_{2}$ detectors. Each cell also has a liquid expansion reservoir which is mounted on the top of the platform. A top view of a $D_{1}$ cell is shown in Figure 6.

Each cell has aluminum side walls cut out for mounting the fused silica windows to which the PMTs are attached. The top and bottom covers of the cell are made of aluminum honeycomb $\left(\approx 250 \mathrm{mg} \mathrm{cm}^{-2}\right)$ to minimize the attenuation of the gamma rays. The interior of the cell is coated with a special white paint compatible with the NE 213A liquid. Since the NE 213A must not contain any bubbles, a bellows-driven expansion chamber is attached to the cell to keep it completely filled with liquid over the expected range of temperatures in flight. A small light-emitting diode for in-flight calibration and testing is sealed into an orifice on the top of the cell. Redundant seals are used throughout the cell assembly to contain the liquid scintillator.

Each PMT assembly is contained in an aluminum cylindrical can. A $\mu$-metal shield surrounds the PMT to minimize the gain changes due to geomagnetic field changes during flight. The bleeder string for the PMT, which is operated at about $1300 \mathrm{~V}$, is mounted on a board attached to the PMT.

The eight PMTs on the cell are operated from one HVPS. A HVJB distributes the proper voltage to each PMT on a particular cell. The HVPS is a standard model common to the COMPTEL subsystems which is settable over the operating range from 900 to $1600 \mathrm{~V}$.

The FEE for each cell provides the amplification of the individual PMT signals, a sum signal, a pulse-shape signal, and a timing trigger. The dynamic range is from $30 \mathrm{keV}$ to $20 \mathrm{MeV}$. The anode signal of each PMT is both summed and processed by a FEE. They are used to derive the energy deposits in a $D_{1}$ cell and to determine the interaction location within the cell (according to the Anger camera principle). From the summed outputs of the last dynode of the eight PMTs a fast-logic signal for pulses above the lower level discriminator is generated. The fast-logic signal is used for coincidence processing, time-offlight measurements, module identification, and module-rate measurement. The levels of the discriminators can be set to eight different values ranging from about 30 to $150 \mathrm{keV}$. Pulseshape discrimination is used in the liquid scintillators to discriminate between the pulses from proton and electron ionization which result from scattering of gamma rays or neutrons, respectively. For this FEE has a fast circuit which is tuned to provide the best pulse-shape discrimination over the specified energy range. Each FEE is adjusted to match the gain and response of the cell to which it is mated.

The platform on which the seven $D_{1}$ modules are mounted is manufactured from a $76 \mathrm{~mm}$ thick plate milled to $3 \mathrm{~mm}$

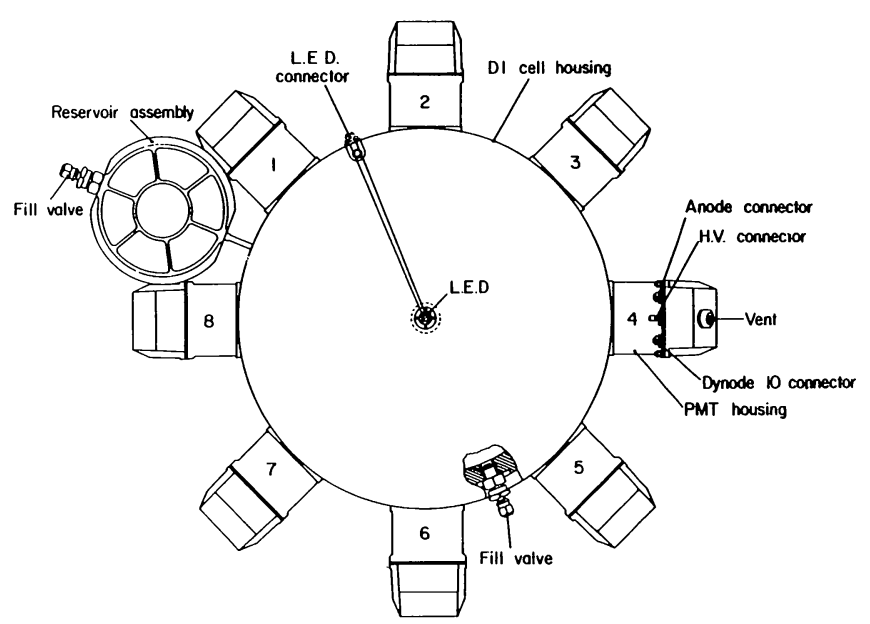

FIG. 6.-Top view of a $D_{1}$ module 


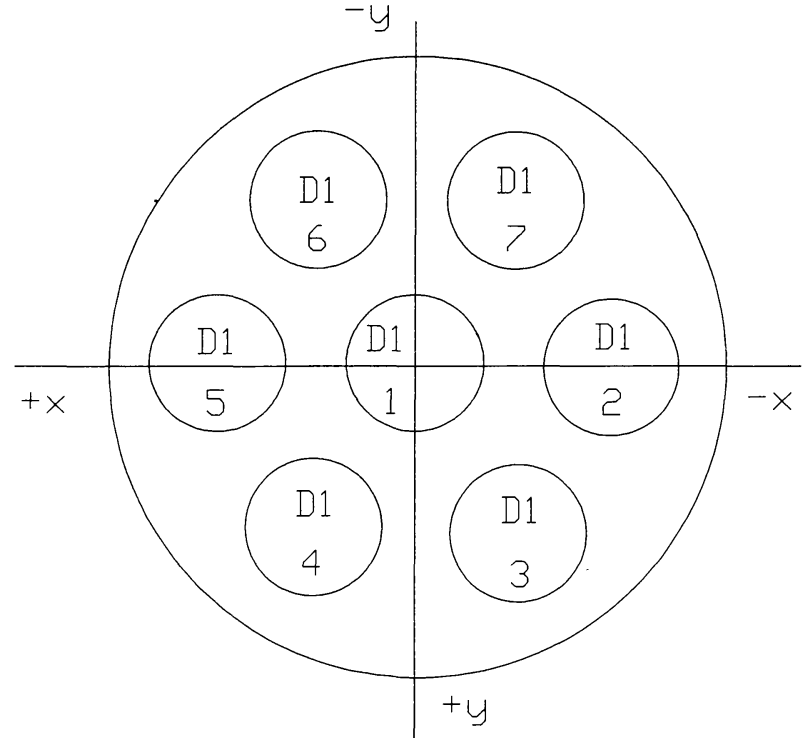

FIG. 7.-Top view of $\mathrm{D}_{1}$ detector. Definition of coordinates $x$ and $y$ is given in Fig. 1.

thickness. The cells are mounted above circular holes cut in the plate. By using thin material the mass adjacent to the cell is reduced, minimizing local production of gamma rays. The passive material above and below a cell is $0.59 \mathrm{~g} \mathrm{~cm}^{-2}$. A top view of the $D_{1}$ detector is shown in Figure 7. The total geometrical area is $4188 \mathrm{~cm}^{2}$. The mass of each $\mathrm{D}_{1}$ module is $15.3 \mathrm{~kg}$. The total mass of the $\mathrm{D}_{1}$ detector is $167.5 \mathrm{~kg}$.

\section{2. $\mathrm{D}_{2}$ Detector Assembly}

The lower detector assembly of COMPTEL, also called the $\mathrm{D}_{2}$ detector assembly, is composed of 14 identical detector modules of cylindrical $\mathrm{NaI}(\mathrm{Tl})$ crystals with a diameter of 28.2 $\mathrm{cm}$ and a thickness of $7.5 \mathrm{~cm}$. A side view is shown in Figure 8 .

Each crystal is hermetically sealed in an aluminum housing. The bottom plate of this housing has seven openings, each having a diameter of $7.6 \mathrm{~cm}$, into which seven $12 \mathrm{~mm}$ thick quartz-glass windows were glued. To these windows EMI 9754 NA photomultiplier tubes are optically coupled and view the crystal from below. The PMTs are enclosed in a light-tight aluminum housing. They are shielded against magnetic fields by $0.25 \mathrm{~mm}$ thick $\mu$-metal. Another $\mu$-metal shield is also glued to the inner sides of the crystal housing, so that each $\mathrm{D}_{2}$ module is completely surrounded by a $\mu$-metal shield. In order to minimize matter in the gamma-ray path, however, the $\mu$-metal beneath the top sandwich plate is only $0.1 \mathrm{~mm}$ thick. To further minimize attenuation, the top plate is made of a $20 \mathrm{~mm}$ thick honeycomb sandwich plate. The area density of the sandwich plate and of the $\mu$-metal shield is $0.219 \mathrm{~g} \mathrm{~cm}^{-2}$.

In addition to the seven large openings, a small hole $(9 \mathrm{~mm}$ in diameter) was drilled through the bottom plate and sealed with a quartz-glass window. Into this hole a light-emitting diode (LED) was mounted for calibration purposes.

On the rear side of the PMT housings of each $\mathrm{D}_{2}$ module is mounted the FEE box and the high-voltage power supply housing. The anode signals of the seven PMTs are summed and individually processed by the FEEs. As in $\mathrm{D}_{1}$, they are used to derive the energy deposited and to determine the interaction location of the event. From the summed outputs of the last

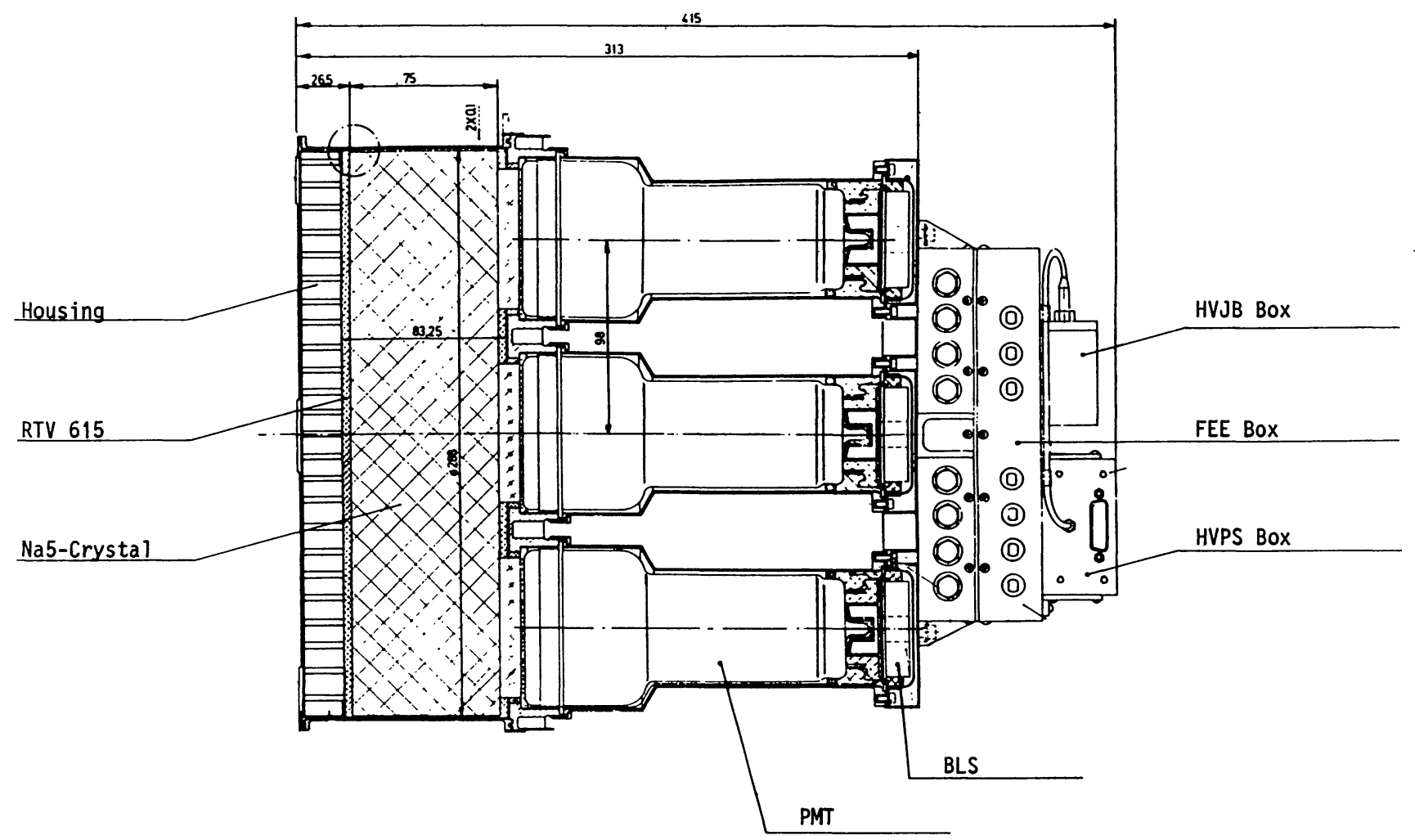

Fig. 8.-Side view of a $D_{2}$ detector module 
dynodes of the seven PMTs a fast-logic signal is generated. The dynamic range is $300 \mathrm{keV}$ to $30 \mathrm{MeV}$. The levels of the discriminator can be set to eight values ranging from $370 \mathrm{keV}$ to 2.3 $\mathrm{MeV}$. The fast-logic signal is used for coincidence processing, time-of-flight measurements, module identification, and module-rate measurement.

The total geometrical area of the 14 cylindrical crystals is $8744 \mathrm{~cm}^{2}$. A top view of the entire $\mathrm{D}_{2}$ detector, is shown in Figure 9 . Each single $\mathrm{D}_{2}$ module is mounted below a supporting sandwich plate with a low-matter density of $0.9 \mathrm{~g} \mathrm{~cm}^{-2}$.

The mass of a single $\mathrm{D}_{2}$ module is $28.2 \mathrm{~kg}$, and the mass of the whole $\mathrm{D}_{2}$ detector is $429.1 \mathrm{~kg}$.

\subsection{Veto Domes}

The anticoincidence subsystem of COMPTEL consists of four veto-dome assemblies. The main part of each veto-dome assembly is a Cassini-shaped dome of a $1.5 \mathrm{~cm}$ thick plastic scintillator (NE 110) with a cylindrical extension at the open end. Each of the two detectors of COMPTEL $\left(D_{1}\right.$ and $\left.D_{2}\right)$ is completely surrounded by two of these domes. In order to allow an overlap, these two domes have different sizes: a large dome fits over each detector from above, with a smaller shield below. The arrangement of the veto domes with respect to the two detectors is shown in Figure 10.

Each of the four veto domes is viewed by 24 photomultiplier tubes (RCA C7151Q). The PMTs are optically coupled to the cylindrical edge of the dome and are enclosed in a light-tight aluminum housing and shielded against magnetic fields by a $0.2 \mathrm{~mm}$ thick $\mu$-metal shield. The optical couplant is RTV 615 . The holding flange made from aluminum also supports the veto dome and the hard covers which surround the plastic scintillator on both sides.

The $0.7 \mathrm{~mm}$ thick hard covers are made of Kevlar and reinforced resin (CY 209/HT 972) which has a density of $1.4 \mathrm{~g}$ $\mathrm{cm}^{-3}$. The sides which face the scintillator are coated with a

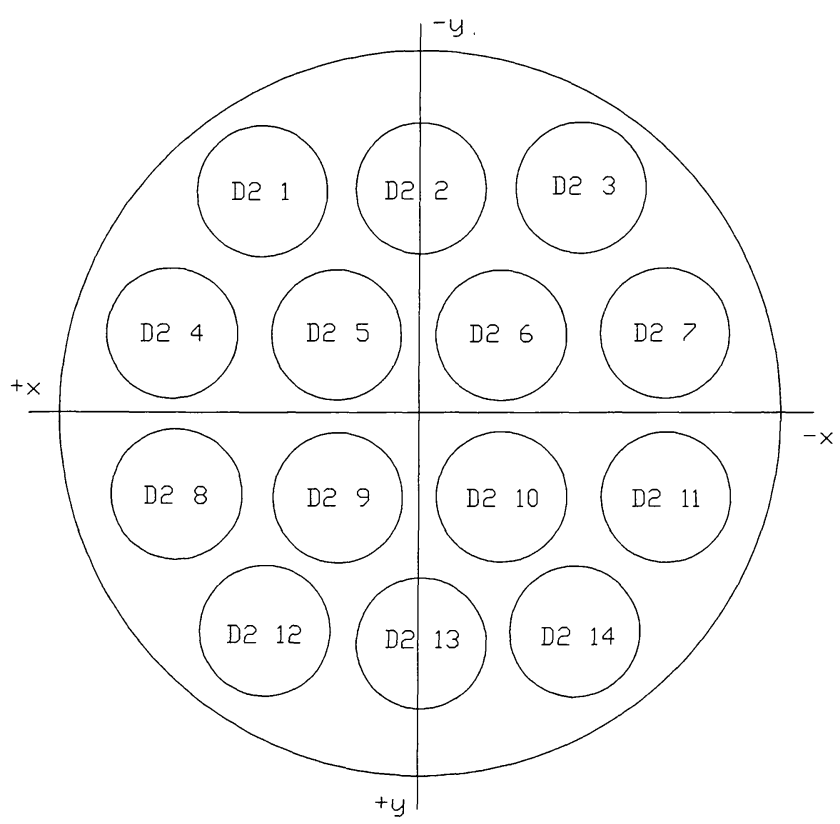

FIG. 9.-Top view of $\mathrm{D}_{2}$ detector white diffuse-reflecting Teflon foil, while the outer surfaces are painted black. The hard covers shield the plastic scintillator and the PMTs from light.

The electronics boxes are mounted on the circular holding flange and between the PMTs. Two front-end electronic units (main and redundant) are attached to each veto-dome assembly, each of which consists of three different boxes and six high-voltage power-supply boxes with the corresponding junction boxes. Each HVPS powers four PMTs. These four PMTs are located in opposite quadrants.

The signals of the 24 PMTs are guided in a cross-strapped manner to the main and redundant FEEs, yielding two sum signals, each of which is processed by its own low-level discriminator (whose level can be set to eight distinct values ranging from about $150 \mathrm{keV}$ to about $1.5 \mathrm{MeV}$ ). The fast-logic output signal of these discriminators is used by the fast-coincidence circuitry (FCC) for anticoincidence processing. The length of the fast-logic signals depends on the energy of the penetrating charged particle and can vary from 60 to 200 ns. All the veto rates are gated by $50 \mathrm{~ns}$ pulses at $312.5 \mathrm{kHz}$. The resulting clock rate is a measure of the dead time.

On the top of each veto dome is a light-emitting diode ( $1 \mathrm{~N}$ 6093 ). The calibration LED is fixed in a $1 \mathrm{~cm}$ thick rounded piece of NE 110 which is glued to each dome. It is used for functional checks during flight.

The mass of a large veto dome is $114.2 \mathrm{~kg}$, and that of a small veto dome is $73.9 \mathrm{~kg}$. In total, the mass of the whole anticoincidence subsystem is $376.2 \mathrm{~kg}$.

\subsection{Calibration Units}

To maintain the energy, angular, and positional resolution of COMPTEL, it is necessary to monitor and adjust the energy response of both the $D_{1}$ and $D_{2}$ modules. There are several effects which can change the gain of a module: loss of transparency, reduced reflectivity, deterioration of optical coupling and gain variation of the photomultipliers, and front-end electronics. A system is built into COMPTEL which provides continuous monitoring of the gains of the system by means of two tagged gamma-ray calibration sources (CALs). Since the events are tagged, they may be treated separately by the onboard data-handling electronics (digital electronics).

The calibration system has been described by Snelling et al. (1986). It is comprised of ${ }^{60} \mathrm{Co}$ doped scintillators, each viewed by two $\frac{1}{2}$ inch $(1.25 \mathrm{~cm})$ PMTs, placed midway between $D_{1}$ and $D_{2}$ out of the gamma-ray light path (see Fig. 10). The scintillation wafer is almost completely surrounded by the PMT photocathode, thus permitting almost $100 \%$ probability of detecting the beta particle's scintillation when the ${ }^{60} \mathrm{Co}$ decays to emit two gamma rays (with energies of 1.17 and 1.33 $\mathrm{MeV}$ ). A low tagging efficiency could not be tolerated, as this could lead to an unwanted contribution to the gamma-ray background. A coincidence between a tagging pulse and a $D_{1}$ gamma event defines a CAL- 1 event, a tagged $D_{2}$ event is a CAL-2 event, and a CAL-3 is a triple coincidence of tagged $D_{1}$ and $\mathrm{D}_{2}$ events.

The strengths of the sources were selected to give enough events in each cell to monitor the gain over time scales of minutes rather than hours, while not burdening the telemetry and on-board electronics with nonastrophysical events. 


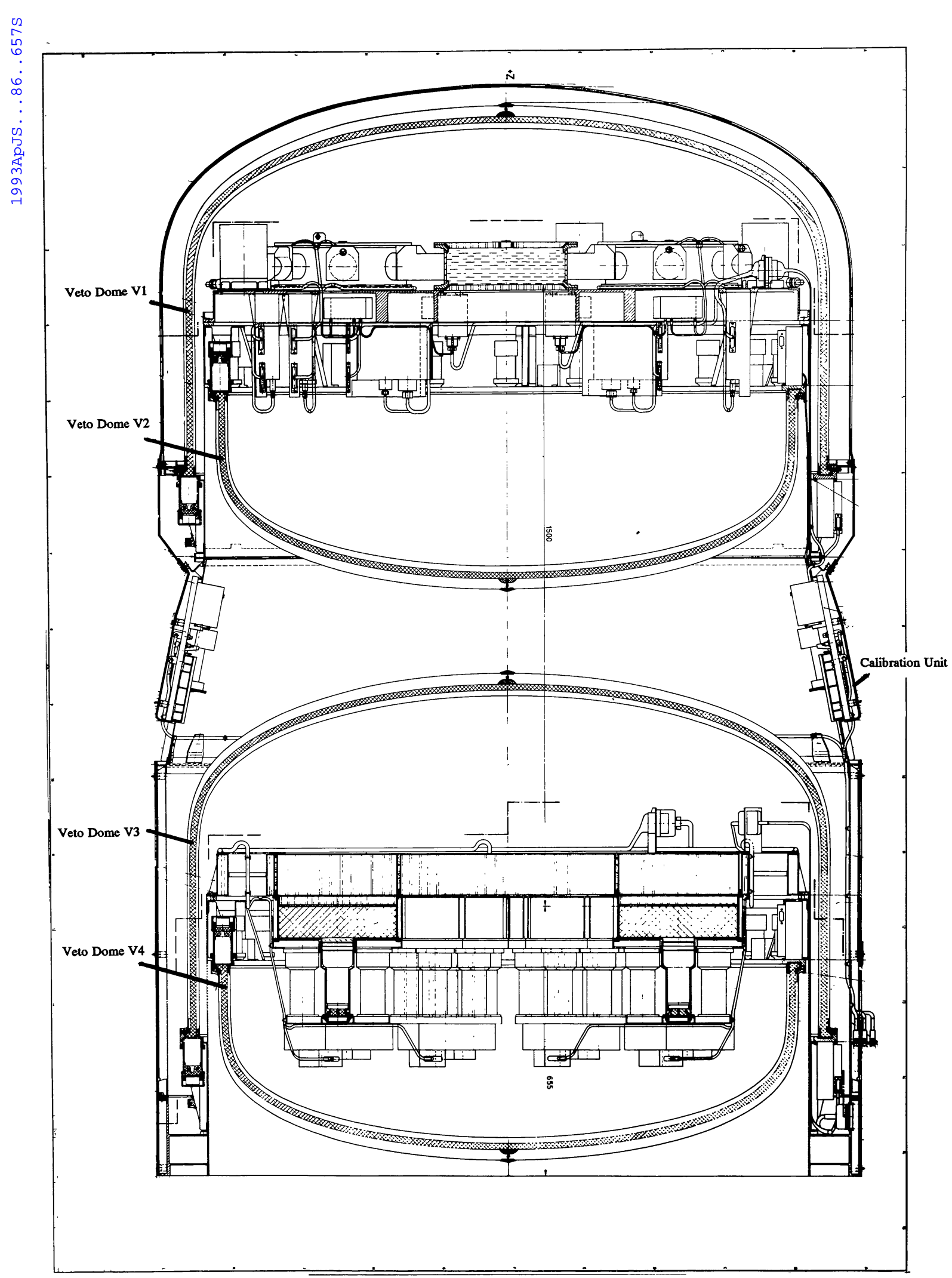

FIG. 10.-Side view of the four veto domes 
The strengths of the calibration sources are given in Table 1. The sources produce about $27 \mathrm{~Hz}$ of CAL-1 events and $33 \mathrm{~Hz}$ of CAL-2 events at the time of the launch. The rate of events in $\mathrm{D}_{2}$ is artificially reduced by about $50 \%$ favoring $\mathrm{D}_{1}$ events which have a broader spectrum requiring greater statistics.

The normal mode of in-orbit operation is to accumulate an average pulse height of the CAL events per PMT. After a preset interval, e.g., 10 minutes, the average pulse-height matrix is transmitted. In orbit the only CAL event data transmitted are the threefold coincidence events (CAL-3).

Although only giving gain information in the low-energy range of COMPTEL, the effective gamma-ray energy for which the system gain is monitored is around the sum energy at $2.5 \mathrm{MeV}$, well above the system threshold. The CAL subsystem also serves as a charged-particle monitor, shutting down high voltage during SAA transits, if COMPTEL is not commanded to do so.

\subsection{Electronics Subsystem}

In Figure 11 a block diagram for COMPTEL shows the main components of the detector and of the remote electronics assembly. Each detector module in the detector assembly is equipped with a front-end electronics box and a high-voltage power supply. For an interaction in a detector module the information about its energy deposit is derived from the anode signal of the photomultiplier tubes. This signal is preamplified for each PMT in the front-end electronics, and in addition the PMT signals of a module are summed. Timing information is derived from the signal of the last dynode through constantfraction discrimination in the FEE. For the $D_{1}$ modules the dynode signal is also used to derive pulse-shape information in order to enable discrimination of neutron-induced interactions. For each detector module threshold levels can be set in the FEE, in eight steps, between 30 and $150 \mathrm{keV}$ for the $D_{1}$ modules and between 370 and $2300 \mathrm{keV}$ for the $D_{2}$ modules.

The remote electronics assembly contains three major components: the analog electronics (AE), the digital electronics (DE), and the low-voltage power supply (LVPS).

The fast-coincidence circuitry in the analog electronics determines the event type based on timing signals from the various detector modules. Events are identified as gamma events in the case of a coincidence between a $D_{1}$ module and a $\mathrm{D}_{2}$ module in anticoincidence with the veto domes, as calibration events in the case of a coincidence between $a D_{1}$ and/or a $\mathrm{D}_{2}$ module and a calibration unit, or as an LED event generated by the light-emitting diodes. Any of these conditions can be overruled by setting special operational modes of COMPTEL. The timing signals from the upper and lower detectors are also used for the time-of-flight measurement. Only events

TABLE 1

Calibration Unit SOURCE STRENGTHS

\begin{tabular}{|c|c|c|c|}
\hline \multirow[b]{2}{*}{ UNIT } & \multirow[b]{2}{*}{$\begin{array}{c}\text { TAGGING } \\
\text { EFFICIENCY }\end{array}$} & \multicolumn{2}{|c|}{ STRENGTH (nCi) } \\
\hline & & 1991 April 30 & 1996 April 30 \\
\hline Calibration A . & $98 \%$ & $20.2 \pm 1.5$ & $10.5 \pm 0.7$ \\
\hline Calibration B ...... & $98 \%$ & $16.8 \pm 1.5$ & $8.7 \pm 0.7$ \\
\hline
\end{tabular}

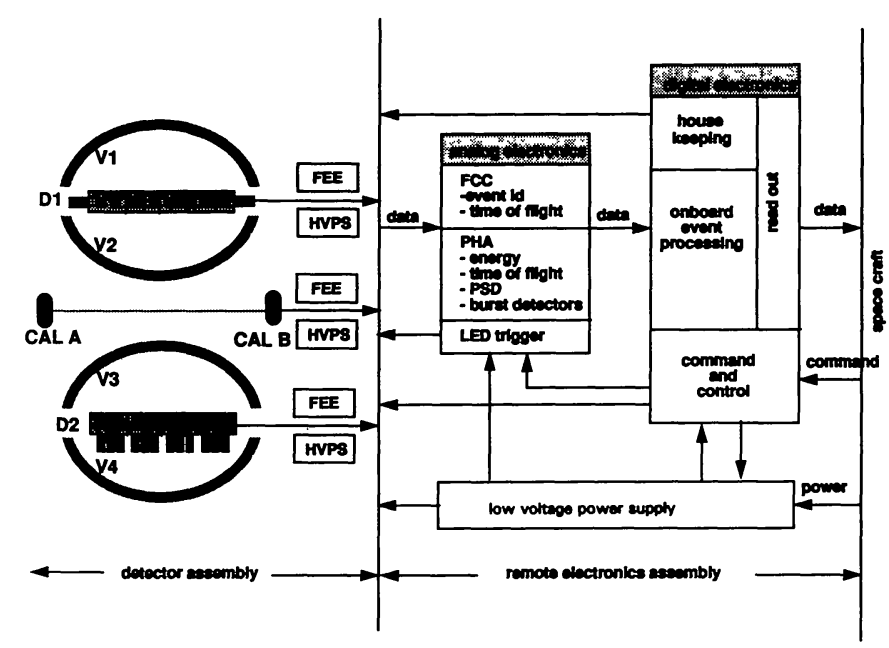

FIG. 11.-Electronic block diagram including the main detector components.

within a 40 ns time-of-flight window are processed. Based on the event identification, the FCC controls the processing and digitization of the energy signals in the pulse-height analysis (PHA) section of the analog electronics. To exploit the full dynamic range of the detectors, the signals are digitized into low and high ranges. The energy signals of the two burst modules are, in addition, processed through completely independent channels (see next paragraph!).

In the digital electronics the on-board selection of events takes place in order to optimize the telemetry. For this purpose, windows on all the major event parameters are available as well as on the ratio of the energy deposits in the $D_{1}$ and $D_{2}$ detectors, a measure of the scatter angle. The DE packs the event data and housekeeping parameters, including all the rate meters, into the spacecraft data stream. Furthermore, the DE controls the instrument through interpretation and distribution of the commands.

The LVPS not only converts the spacecraft power into the secondary voltages but also serves as a signal distribution and switching box. The electronics for each individual detector module, main and redundant sections, as well as the HVPSs, can be switched on and off.

With a packet size of 799 words the event transmission rate is limited to 48 events per packet or each $2.048 \mathrm{~s}$. It was an important task of the early mission to ensure that the limited telemetry was used for the most astrophysically important events. This was achieved by methodical instrument tuning during which all orbital variations and event-rate variations were monitored to provide the optimum settings for COMPTEL.

The different event types can be selected by the digital electronics as summarized in Table 2. Gamma-1 events are defined by energy windows in $\mathrm{D}_{1}$ and $\mathrm{D}_{2}\left(E_{1}>50 \mathrm{keV}, E_{2}>500\right.$ $\mathrm{keV}$ ) and by a time-of-flight (TOF) window ( $-7 \mathrm{~ns}<\mathrm{TOF}<$ $+8 \mathrm{~ns}$ ); no constraints are put on the pulse-shape value or on the ratio of energy losses in $D_{1}$ and $D_{2}$. Gamma- 2 events are those which fall outside the windows for gamma-1 events $\left(E_{1}>35 \mathrm{keV}, E_{2}>440 \mathrm{keV} ;-23 \mathrm{~ns}<\mathrm{TOF}<20 \mathrm{~ns}\right)$. The CAL events are selected by energy loss windows in $D_{1}$ and $D_{2}$. 
TABLE 2

COMPTEL EVENT TYPES

\begin{tabular}{|c|c|c|c|c|}
\hline Event Type & Description & Rate & $\begin{array}{l}\text { Transmission } \\
\text { Priority }\end{array}$ & Use \\
\hline Gamma-1 ......... & $\begin{array}{l}\text { First-quality double-scatter } \\
\text { events }\end{array}$ & $<20 \mathrm{~Hz}$ (tuneable) & 1 & Astrophysics \\
\hline Gamma-2 ......... & $\begin{array}{l}\text { Second-quality double- } \\
\text { scatter events }\end{array}$ & $20 \mathrm{~Hz}$ minus gamma- 1 rate & 2 & $\begin{array}{l}\text { Background evaluation and } \\
\text { solar neutrons }\end{array}$ \\
\hline Gamma-3 & $\begin{array}{l}\text { Rejected double-scatter } \\
\text { events }\end{array}$ & $1 \mathrm{~Hz}$ & Selectable & Background evaluation \\
\hline CAL-3 $\ldots \ldots \ldots \ldots$ & ${ }^{60} \mathrm{Co}$ coincidence events & $1 \mathrm{~Hz}$ & 1 & Calibration \\
\hline CAL-1/CAL-2 ...... & ${ }^{60} \mathrm{Co}$ single-detector events & $\sim 40 \mathrm{~Hz}$ & $\begin{array}{l}\text { Processed on } \\
\text { board }\end{array}$ & Calibration \\
\hline CAL-4/CAL-5 ...... & LED-stimulated events & $0-32 \mathrm{~Hz}$ & 1 & Calibration \\
\hline
\end{tabular}

\subsection{Single-Detector Burst-Mode Description}

COMPTEL uses two of its $14 \mathrm{D}_{2}$ modules to accumulate burst spectra upon receipt of an external trigger signal from the BATSE instrument. The modules are in principle sensitive over $4 \pi$ sr. However, the field of view is not completely free of intervening material: within a range of zenith angles $0^{\circ} \leq \theta \leq$ $45^{\circ}$ ( $\theta$ measured from $G R O z$-axis $)$ the field of view of the two burst detectors is obstructed by the $\mathrm{D}_{1}$ detector array, the veto domes $V_{1}-V_{3}$, and the sandwich plate supporting the $D_{2}$ assembly. For $\theta \geq 45^{\circ}$ the obstruction is more severe due to the spacecraft, other GRO instruments (OSSE, EGRET), and electronics boxes.

The two modules measure two different energy ranges: module $\mathrm{D}_{2}-14$ covers the low range $(\approx 50 \mathrm{keV}$ to $1.1 \mathrm{MeV})$ with 128 channels, and $\mathrm{D}_{2}-7$ the high range ( $\approx 160 \mathrm{keV}$ to $11 \mathrm{MeV}$ ) with 128 channels. The low-range threshold in $D_{2}-14$ is the result of the absorption of gamma rays in the veto domes and the sandwich plate. The modules are equipped with dedicated analog-to-digital converters (ADCs) and an electronic subsystem (burst spectrum analyzer [BSA]) (Winkler et al. 1986).

The BSA incorporates two microcomputers, the time and command (TAC) and the BURST processor. The TAC receives and buffers commands from the $G R O$ Remote Interface Unit (RIU) to generate and synchronize the real-time clock, to send time-tag and command-status information to the BURST processor, to buffer spectra from the BURST processor, and to send spectra to the digital electronics. In the DE the data are inserted in the COMPTEL data packet for transmission. The BURST processor accumulates and makes histograms for burst data over 128 channels per module (energy range), integrates over a selectable time interval (the maximum number of counts is 65,535), appends a label consisting of a time tag and command-status information to the spectrum, generates test patterns on receipt of command, and generates time integration intervals and hold-off times. The label consists of six words overwriting the six lowest channels in $\mathrm{D}_{2}-14$.

The BSA operates in four different modes (Fig. 12): The background mode is the normal mode. Spectra from both modules are accumulated over a telecommandable period of time (2-512 s per spectrum) and stored in the first two memory blocks of the BURST processor. The data are read out continually or at a reduced (telecommandable) rate, e.g., every 5 minutes. Data from these spectra are used to investigate the total (instrumental + astrophysical) background before and after the burst event. The BSA switches into the burst mode after receipt of an external trigger signal from BATSE. The integration time per spectrum in the burst mode can be preselected from $0.1 \mathrm{~s}$ to $25.6 \mathrm{~s}$; six burst-mode spectra from each energy range are accumulated. After completion of the sixth pair of spectra the BSA enters the tail mode automatically, where up to 255 spectra with an individual integration time of 2-512 s are accumulated and read out. This tail mode was incorporated to look for both repetitive bursts and long-duration solar flares. After completion of the tail mode, the BSA enters the background mode. A test mode generates triangularshaped spectra to test the integrity of the random access memory (RAM). A burst hold-off time can disable the burst trigger for a predefined interval following the burst-tail sequence. A background hold-off indicates the time that is to elapse before a background spectrum is transmitted. The standard configuration for the BSA (nominal operation) is shown in Table 3.

\subsection{Other Modes of Operation}

The operation of COMPTEL is under control of the digital electronics. Major main and redundant units are switched by relay command. Other modes are selected by means of bilevel controls from the digital electronics steered by serial commands.

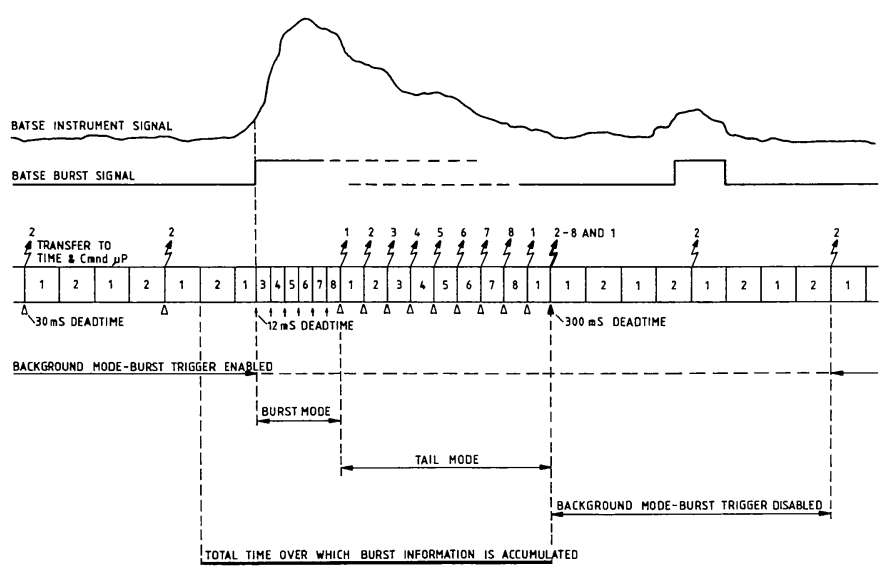

FIG. 12.-Burst sequence showing COMPTEL's modes of burst operation. 
TABLE 3

CONFIGURATION OF BURST SPECTRUM ANALYSIS FOR EARLY IN-FLIGHT OPERATIONS

\begin{tabular}{ll}
\hline \multicolumn{1}{c}{ Parameter } & \multicolumn{1}{c}{ Value } \\
\hline Background integration time $\ldots \ldots \ldots \ldots \ldots \ldots$ & $100 \mathrm{~s}$ \\
Burst integration time $\ldots \ldots \ldots \ldots \ldots \ldots \ldots \ldots$ & $0.5 \mathrm{~s}$ per spectrum \\
Tail integration time $\ldots \ldots \ldots \ldots \ldots \ldots \ldots \ldots$ & $6 \mathrm{~s}$ per spectrum \\
Numbered tail spectra $\ldots \ldots \ldots \ldots \ldots \ldots \ldots$ & 100 \\
Hold-off times (background, burst) $\ldots \ldots \ldots$ & $900 \mathrm{~s}$ \\
\hline
\end{tabular}

By judicious use of the instrument control, several standard modes of operation have been defined with the objective of obtaining diagnostic information when the astrophysical source is occulted and maximum sensitivity during unocculted periods. These modes are briefly described in Table 4 .

The normal mode of operation is the double-scatter telescope mode with the gamma-1 and gamma-2 event selections as defined in $\S 3.5$. The only other astrophysical mode is the solar neutron mode, which allows measurements of solar neutrons in the double-scatter mode on a command stream sent in response to a BATSE signal to the on board computer. All other modes are for calibration purposes only.

All modes are achieved by scheduled command loads except for solar neutrons. This mode is entered when the BATSE decides a gamma burst originated from the direction of the Sun. As this mode is unsolicited and may occur during another mode, an independent overriding-mode flag is provided. By means of the mode flags the COMPTEL Processing and Data Analysis System is able to sort the modes into different data sets.

\subsection{Mechanical Structure and Thermal Control}

The mechanical structure is comprised of the upper and lower structure, the $D_{1}$ detector platform, and the $D_{2}$ detector array support plate. It is fabricated from aluminum to minimize the mass around the two detectors, thus reducing the internally produced background radiation. The upper and lower structure consists of hollow cylinders manufactured from thin aluminum sheets reinforced by struts. The two parts of the structure are connected by a conically shaped hollow section to which the calibration units are attached. The dis- tance from the center of the $D_{1}$ detector to the center of the $D_{2}$ detector is $1577 \mathrm{~mm}$. The main electronics (pulse-height analysis, digital electronics, and power supply) are mounted in boxes separate from the telescope on the spacecraft structure (see Fig. 1). The mass of the whole structure is $114.7 \mathrm{~kg}$. COMPTEL's total mass (including the main electronics assembly) is $1324 \mathrm{~kg}$. A cross section through COMPTEL is shown in Figure 10.

In order to comply with temperature specifications, COMPTEL is equipped with a thermal-control subsystem which is both passive and active. It consists of the following elements: (1) multilayer-insulation blankets; (2) a radiator area to dissipate excess heat (via an automatic heat-pipe absorber system); (3) black coatings to enhance radiative heat transfer within the detector assembly of COMPTEL; (4) heater circuits which are automatically controlled by thermostats preventing low temperatures; and (5) thermistors to monitor temperatures. The multilayer insulation consists of 15 aluminized Kapton foils, of which the 13 innermost are $750 \mu \mathrm{m}$ thick whereas each of the two outer ones (of the type Kapton Nomex) is $127 \mu \mathrm{m}$ thick. The top side of COMPTEL is further covered with a $\beta$-cloth and a circle with a diameter of $57.5 \mathrm{~cm}$ around the telescope axis with a second surface mirror (SSM) foil. The operating temperature of COMPTEL ranges from about $+10^{\circ}$ to about $+30^{\circ}$. COMPTEL's total power consumption during normal operation is $144 \mathrm{~W}$.

\section{DETERMINATION OF INSTRUMENT CHARACTERISTICS}

There are three different ways to determine the instrument response:

1. Calibration measurements of the entire telescope using known monochromatic photon sources at specified positions.

2. Calibration measurements of instrument detector modules using known monochromatic photon sources, and convolving of these detector-module responses into a telescope response via (assumed) physical characteristics of the telescope.

3. Calculated simulation of physical processes that are experienced by interacting photons in the telescope.

All three methods have been fully exploited. Each of the methods is described in detail below.

TABLE 4

STANDARD COMPTEL IN-FLIGHT MODES

\begin{tabular}{|c|c|c|}
\hline Mode & Description & Purpose \\
\hline Normal operation mode & Nominal configuration & Astrophysical data \\
\hline Solar neutron mode $. . . \ldots \ldots \ldots \ldots \ldots . . .$. & $\begin{array}{l}\text { Neutron event selection } \\
(0 \mathrm{~ns}<\mathrm{TOF}<40 \mathrm{~ns})\end{array}$ & Astrophysical data \\
\hline Earth albedo mode & View Earth & $\begin{array}{l}\text { Atmospheric data } \\
\text { (gamma rays and neutrons) }\end{array}$ \\
\hline SAA mode $\ldots \ldots \ldots \ldots \ldots \ldots \ldots \ldots \ldots$ & HVPS off during SAA passage & FEE and BSA calibration \\
\hline Proton/charged-particle event mode ....... & Vetos ignored & Veto calibration \\
\hline $\mathrm{D}_{1}$ single-event mode $\ldots \ldots \ldots \ldots \ldots \ldots$ & $\mathrm{D}_{2}$ ignored & $D_{1}$ calibration \\
\hline $\mathrm{D}_{2}$ single-event mode $\ldots \ldots \ldots \ldots \ldots \ldots \ldots$ & $D_{1}$ ignored & $\mathrm{D}_{2}$ calibration \\
\hline LED mode $\ldots \ldots \ldots \ldots \ldots \ldots \ldots \ldots \ldots \ldots \ldots \ldots \ldots$ & High rate & Calibrate cell gains \\
\hline Safe mode $\ldots \ldots \ldots \ldots \ldots \ldots \ldots \ldots \ldots$ & Low voltage only & Safety \\
\hline
\end{tabular}




\subsection{Telescope Mode Calibration}

The entire telescope COMPTEL has been calibrated before launch using gamma-ray and neutron-radiation sources of various types and energies at the accelerator laboratory of the Gesellschaft für Strahlen- und Umweltforschung (GSF) in Neuherberg near Munich, Germany.

The calibration setup at GSF is shown in Figure 13. The instrument was operated with the instrument ground support equipment attached to the instrument via a command interface simulation unit. In order to enhance the data throughput of the recording system, the instrument event recording electronics was attached via a switch to an external data acquisition system which bypassed the packet telemetry system used in flight for event transmission (all other data types were recorded in the same way as during the space mission).

The calibration at GSF resulted in a data volume equivalent to 1.5 years of mission. The calibration photons were generated from radioactive nuclide samples, or directly via nuclear reactions initiated in target materials of different types from the GSF proton Van de Graaff accelerator.

The photon sources listed in Table 5 were used for calibration. The last three calibration sources of Table 5 are protoninitiated nuclear reactions, which have accompanying secondary radiation contaminating the ideal monochromatic calibration source spectra.

For each of these calibration sources a set of incidence angles (see Table 6) was chosen by manipulation of the telescope position with respect to the radiation source; the telescope was mounted on a calibration dolly enabling positioning of the calibration source positions within the instrument field of view by rotations of the instrument about a vertical and a horizontal axis. The source measurements were supplemented by "background" measurements during which the direct source radiation was blocked by a massive lead attenuator, shadowing the instrument from direct calibration radiation.

The distance of the radioactive sources/targets from the $D_{1}$ detector of COMPTEL varied between 8 and $9 \mathrm{~m}$, depending

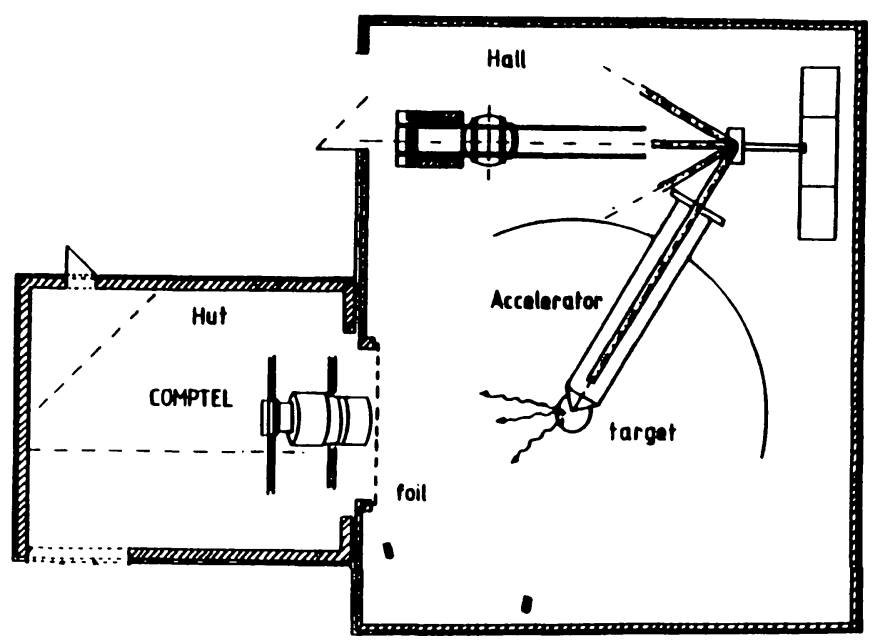

FIG. 13.-Setup of the response calibration measurements at the GSF accelerator site. The instrument was supported by a special calibration dolly. The Van de Graaff proton accelerator was used to initiate nuclear reactions as calibration photon sources.
TABLE 5

\begin{tabular}{|c|c|}
\hline Source & $\begin{array}{l}\text { Photon Energy } \\
(\mathrm{MeV})\end{array}$ \\
\hline${ }^{137} \mathrm{Cs} .$. & 0.662 \\
\hline${ }^{54} \mathrm{Mn} . \ldots \ldots \ldots \ldots$ & 0.835 \\
\hline${ }^{22} \mathrm{Na} \ldots$ & $0.511,1.275$ \\
\hline${ }^{88} Y \ldots$ & $0.898,1.836$ \\
\hline${ }^{24} \mathrm{Na} \ldots \ldots$ & $1.369,2.754$ \\
\hline${ }^{241} \mathrm{Am} /{ }^{9} \mathrm{Be} \ldots \ldots \ldots$ & 4.430 \\
\hline${ }^{19} \mathrm{~F}(p, \alpha \gamma){ }^{16} \mathrm{O} \ldots \ldots$ & 6.13 \\
\hline${ }^{11} \mathrm{~B}(p, \gamma)^{12} \mathrm{C} \ldots \ldots$. & $12.14,16.57,4.43$ \\
\hline${ }^{3} \mathrm{H}(p, \gamma)^{4} \mathrm{He} \ldots \ldots$ & 20.52 \\
\hline
\end{tabular}

on the orientation (see Fig. 13). This distance results in a divergence of the photon beam of $2^{\circ}$ over each $D_{1}$ module, which must be corrected in order to measure the angular resolution of the telescope.

The thermal characteristics of the instrument and its electronics were calibrated during a 1 month long thermalvacuum test of the flight instrument. $\mathrm{A}^{88} \mathrm{Y}$ radioactive source (photon energies 0.898 and $1.836 \mathrm{MeV}$ ) was used for this purpose. In addition to this calibration of the photon response of the instrument, the response to neutrons was also measured, providing information for both neutron detection objectives and background suppression. The response of the instrument to neutrons was measured at GSF via measurements of 17 $\mathrm{MeV}$ neutrons from the ${ }^{3} \mathrm{H}(d, n){ }^{4} \mathrm{He}$ reaction, and via measurement of $6 \mathrm{MeV}$ neutrons from the ${ }^{2} \mathrm{H}(d, n)^{3} \mathrm{He}$ reaction. Background measurements were made with a large paraffin cone shielding the instrument from direct neutrons from the calibration source.

The determination of the response of the instrument to gamma-ray bursts in the single-detector mode required a special calibration effort. The field of view of the two $\mathrm{D}_{2}$ modules used for this purpose is partially occulted or attenuated by the various material components of COMPTEL and the Gamma Ray Observatory. Therefore, a fine grid of measurements at a large set of incidence directions had been performed at GSF to establish the baseline response of the burst system to photon sources of different energies and intensities. After integration

TABLE 6

ENERGIES AND POSITIONS OF CALIBRATION

\begin{tabular}{|c|c|c|}
\hline Source & $\begin{array}{l}\text { Energy } \\
(\mathrm{MeV})\end{array}$ & $\begin{array}{l}\text { Zenith Angles } \\
\text { (degrees) }\end{array}$ \\
\hline${ }^{54} \mathrm{Mn}$ & 0.84 & $02040 * 60$ \\
\hline${ }^{22} \mathrm{Na}$ & 1.27 & $\begin{array}{lllllll}0 & 10 * & 20 & 40 & 60 * & 80 & 102\end{array}$ \\
\hline${ }^{24} \mathrm{Na}$ & 2.75 & $010^{*} 20^{*} 304060^{*}$ \\
\hline${ }^{241} \mathrm{Am} /{ }^{9} \mathrm{Be}$ & 4.43 & $0 \quad 10^{*} 20 \quad 4060$ \\
\hline${ }^{16} \mathrm{O} \ldots \ldots \ldots \ldots$ & 6.13 & 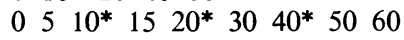 \\
\hline${ }^{12} \mathrm{C} \ldots .$. & 12.14 & $\begin{array}{llllllll}0 & 5 & 10 * & 15 & 20 & 30 & 40 * & 102\end{array}$ \\
\hline Tritium & 20.25 & $015^{*} 40$ \\
\hline
\end{tabular}

NoTE.-At zenith angles followed by an asterisk, more than one azimuthal orientation of the instrument was calibrated. 
of the instrument onto the $G R O$ platform, a set of reference calibrations at critical incidence directions was performed in order to measure the effect of observatory components outside the COMPTEL instrument on the burst system's response.

\subsection{Calibration of Individual Detector Modules}

The calibration of the telescope as a whole is necessarily limited in scope because the entire range of parameters for the incoming radiation cannot be covered. Therefore, the direct telescope calibration must be supplemented by other means, which make use of the knowledge of the instrument detection physics. One approach would be to perform a complete instrument simulation by particle-transport computer programs. Alternatively, one could perform a combination of calibration measurements to determine the characteristics of individual instrument components in detail, and employ these characteristics to construct a model to describe the response of the full instrument. For this purpose, the following components were calibrated in detail, using special measurements:

1. The responses of the scintillation detectors $D_{1}$ and $D_{2}$ by themselves were calibrated using the photon sources of Table 5 in a "single-detector" recording mode (for setup see Fig. 14).

2. The Anger camera location characteristics of each of the 21 main detector modules were calibrated with a gamma-ray pencil beam. The pencil beam was generated using a lead colli-
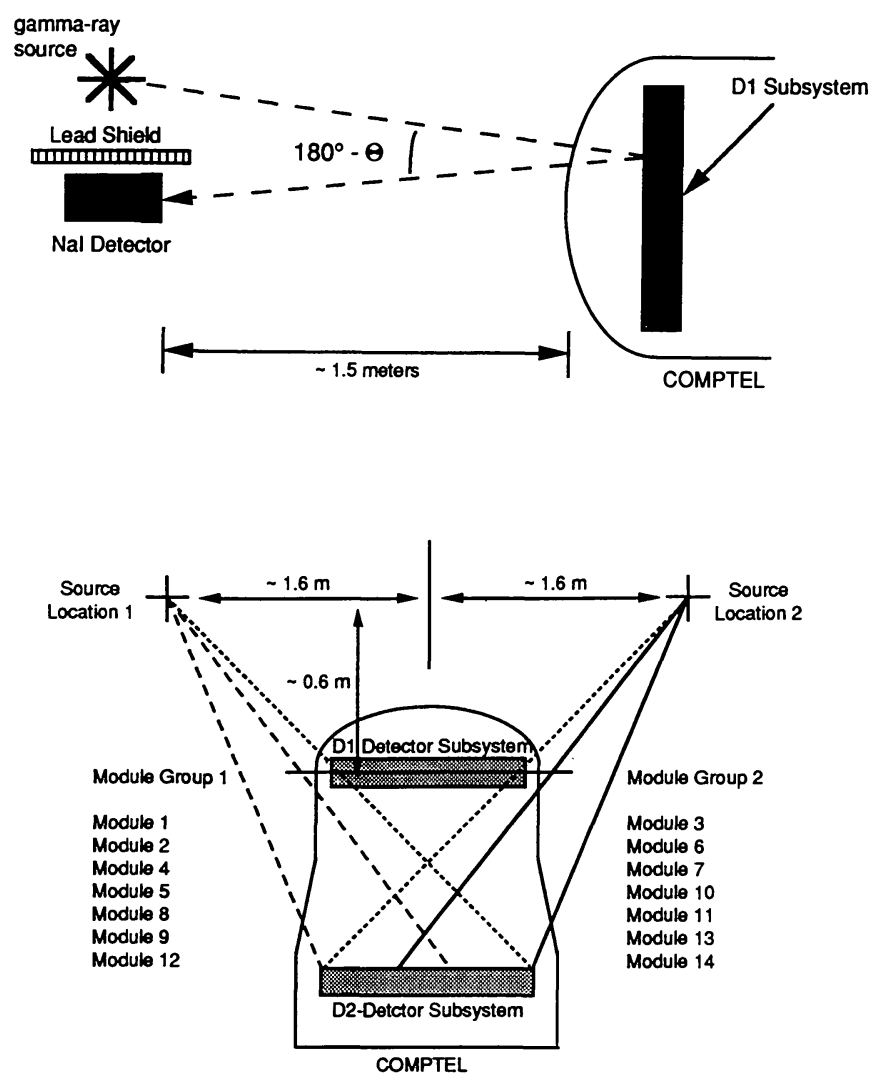

FIG. 14.-Measurement setup for calibration of the single-detector responses. For $\mathrm{D}_{1}$ calibration, a backscatter coincidence technique was used (top view). For $\mathrm{D}_{2}$ calibration the gamma-ray sources were fixed about 1.6 $\mathrm{m}$ off-axis (bottom view). mator and either a ${ }^{22} \mathrm{Na}$ radioactive source (for $\mathrm{D}_{2}$ ) or a ${ }^{60} \mathrm{Co}$ calibration source (for $\mathrm{D}_{1}$ ).

3. The rejection capabilities of the anticoincidence detector system of the four plastic scintillator domes surrounding the $D_{1}$ and $D_{2}$ subsystems were calibrated by operating the $D_{1}$ and $\mathrm{D}_{2}$ detectors as a muon telescope. Muon trajectories through the $1.5 \mathrm{~cm}$ thick scintillator domes yield the specific energy deposit of "minimum ionizing particles" and provide a clean calibration source for the anticoincidence. In order to discriminate precisely against contaminating other high-energy event signatures, the $D_{1}$ and $D_{2}$ coincidence was supplemented by another external scintillation detector to construct a threefold coincidence muon telescope.

4. In addition, calibration measurements had already been performed on the scientific model of COMPTEL, consisting of two $D_{1}$ modules and three $D_{2}$ modules in a laboratory setup referred to as "SM III." The Indiana University cyclotron facility at Bloomington was used to generate nearly monochromatic neutron beams of energies ranging from 35 to $200 \mathrm{MeV}$. The instrument model was exposed to these beams at a variety of different incidence angles ranging from $0^{\circ}$ to $135^{\circ}$.

\subsection{Monte Carlo Simulations}

In order to understand the telescope response better, to augment the calibration, and to investigate differences between the calibration and conditions encountered in flight, a Monte Carlo simulation is employed (Kippen 1991). The simulation consists of two parts: one simulates photon and particle interactions using a model, which represents COMPTEL's material and geometrical properties (the mass model), while the other describes the inherent measurement resolutions and thresholds (the response model).

The mass model, using the GEANT software package from CERN (Brun et al. 1987), is an approximation of the detector mass distribution which accurately reproduces the total instrument mass. It consists of two detector assemblies, each surrounded by simplified veto domes and an idealized support structure. Within the detector assemblies, the active volume of each detector module is modeled with its housing (aluminum with quartz windows ) and attached photomultipliers; in the $D_{1}$ assembly the seven liquid-scintillator reservoirs have also been modeled. Particular care has been taken to reproduce accurately the mass above the $D_{1}$ detector and along the lines of sight between the $D_{1}$ and $D_{2}$ modules.

The simulation of each event is initiated with the selection of an initial photon energy and trajectory. Two restrictions are placed on these initial parameters to reduce computation time. First, the initial photon positions are at the upper surface of the $\mathrm{V}_{1}$ anticoincidence dome, avoiding propagation from the source. Second, only trajectories which intersect the active volume of a $D_{1}$ module are considered. $A$ few simulations have been done without this second restriction, to demonstrate that it has a negligible effect on the derived telescope response.

Each photon is propagated through the mass model, simulating interactions including pair production, Compton scattering, and photoelectric absorption. For secondary electrons the discrete interactions simulated include bremsstrahlung, Møller scattering, and positron annihilation; interactions with energy loss less than $10 \mathrm{keV}$ are approximated by ionization 
loss and multiple scattering in each propagation step. Ionization losses together with the residual energy of particles falling below a $10 \mathrm{keV}$ cutoff are accumulated for each body in the mass model as the energy deposit in that body.

The standard output for each simulated telescope event consists of nine quantities: identification of the $D_{1}$ and $D_{2}$ modules involved, locations of the initial interactions in each module, the time interval between the initial interactions in the two modules (TOF), and energy deposits in the two modules and the four anticoincidence domes. The response model can then be used to modify the interaction locations and module energy deposits to simulate the module resolution. This is done by adding an "error," randomly chosen from a normal distribution, to each of these quantities; the standard deviation of each normal distribution is based on the module resolution as determined in the module calibration. A single, mean value of the location resolution is used for each type of module $\left(D_{1}\right.$ or $\left.D_{2}\right)$, neglecting variations in resolution with location and energy deposit; the location broadening is not allowed to place events outside a module. The simulated energy resolution for each $D_{1}$ module is derived from a least-squares fit to module calibration results of the form

$$
\sigma_{E_{1}}=A+B E_{1}^{1 / 2}
$$

where $E_{1}$ is the unbroadened $\mathrm{D}_{1}$ energy deposit. The fit parameters $A$ and $B$ are listed for each $\mathrm{D}_{1}$ module in Table 7 .

Similarly, the simulated $D_{2}$ energy resolution is derived from a least-squares fit to module calibration results below 10 $\mathrm{MeV}$ of the form

$$
\sigma_{E_{2}}=\left(A E_{2}+B E_{2}^{2}\right)^{1 / 2}
$$

where $E_{2}$ is the unbroadened $\mathrm{D}_{2}$ energy deposit; the first term accounts for statistical variance in the number of photons detected, while the second is due to variance in the transfer efficiency from the point of photon creation to the first photomultiplier diode. The composite fit parameters (for all 14 modules) are $A=1.28$ and $B=3.6$, where $E_{2}$ is measured in kilo-electron volts. The response model also rejects events based on the energy thresholds in the modules and anticoincidence domes. Each module threshold is modeled by an acceptance probability varying linearly from 0 to 1 between two points near the nominal threshold; the two points were deter-

TABLE 7

$D_{1}$ ENERGY-RESOLUTION FIT-FUNCTION PARAMETERS FOR EACH MODULE ${ }^{\mathrm{a}}$

\begin{tabular}{lcc}
\hline \hline $\mathrm{D}_{1}$ Module & Parameter $A$ & Parameter $B$ \\
\hline $1 \ldots \ldots \ldots \ldots$ & -1.022 & 1.749 \\
$2 \ldots \ldots \ldots \ldots$ & -7.481 & 2.157 \\
$3 \ldots \ldots \ldots$ & +2.944 & 1.723 \\
$4 \ldots \ldots \ldots$ & -5.822 & 1.935 \\
$5 \ldots \ldots \ldots$ & -3.073 & 1.892 \\
$6 \ldots \ldots \ldots$ & -8.662 & 2.069 \\
$7 \ldots \ldots \ldots$ & +0.020 & 1.890 \\
\hline
\end{tabular}

a The fit function is $\sigma_{E}=A+B E^{1 / 2}(E$ is in units of $\mathrm{keV})$. mined for each module based on module calibration data. Events can also be rejected if the energy deposit in any anticoincidence dome, multiplied by the fraction of active mass in the modeled dome, exceeds a threshold determined from muon measurements; the modeled anticoincidence dome masses included hard covers, thermal insulation, and micrometeorite shield material, as well as the plastic scintillator. Events in which the first interaction occurs in $D_{2}$ (negative TOF) are also rejected.

Finally, the response model can also apply "software" selections based on the module energy deposits, total energy deposit, and scatter angle $(\bar{\varphi})$. The selected events are used to estimate the telescope efficiency and resolutions in energy and angle. Examples of such results, based on 10,000 simulated telescope events with energy deposits above threshold at each incident energy and angle, are presented below in comparison with the calibration ( see $\S 6$ ). Given the good agreement between the simulation and the calibration, the simulation can be used with confidence to determine the instrument response at energies and angles not covered in the calibration or to determine the response to astronomical sources.

Two other simulations are also being developed to study questions for which the GEANT model of COMPTEL is inadequate. The GEANT code does not accurately simulate interactions of low- and intermediate-energy neutrons. Therefore, a second mass model of COMPTEL is being developed with the MCNP code (Forster et al. 1990; O'Neill et al. 1992) to determine the response to neutrons; this model will be as similar to the GEANT model as possible. A mass model of the entire $G R O$ spacecraft is also being developed by all four experiments using the HETC, MORSE-CG, and EGS4 codes from Oak Ridge National Laboratory. The GRO mass model will be used together with the GEANT mass model to study the response to various backgrounds affected by scattering and other interactions in the spacecraft. Finally, none of the simulation software packages simulate polarized radiation; to determine COMPTEL's response to polarized radiation, the GEANT code will be modified to simulate Compton scattering of polarized radiation.

\section{PERFORMANCE OF MAIN DETECTOR ELEMENTS}

\subsection{Energy Loss Assignment and Gain Corrections}

The energy calibration of the $D_{1}$ and $D_{2}$ detector modules was performed in three steps: $(a)$ the conversion of the recorded pulse-height analysis channel numbers into an output of the PMTs in volts; $(b)$ handling of the PMT gain variations between the different calibration measurements; and $(c)$ the calibration of the PMT output (volts) into energy using known radioactive sources.

Each detector module is monitored by eight $\left(D_{1}\right)$ or seven $\left(D_{2}\right)$ PMTs. For every event the digitized output of the PMTs as well as the summed signal (analog sum) is recorded in either the low range or, when it is above a certain energy, in the high range. The difference between the two ranges is an amplification factor of about 8 . The calibration of these two ranges must be consistent. Using gamma-ray events, the channel-to-volt conversion was fitted under the constraint that there should be no difference between the digital and the analog sum. Comparison of different fit functions indicated that the electronics can 
be described by a linear function and that there is no discontinuity between the low and high ranges. Using the same approach, the stability of the electronics was checked over a longer period. There were no significant changes over a time scale of several months. Furthermore, the dependence on temperature proved to be negligibly small. During a period of more than 2 years, small changes (about $1 \%$ ) were observed. These can be corrected using the redundant information (analog sum) as discussed above.

COMPTEL contains 156 PMTs in its 21 detector modules. The PMTs are operated with a fixed high voltage, and no active gain corrections are made. For the energy calibration, off-line gain corrections had to be applied, since there were gain variations between the different measurements. These corrections are determined using photons from the two ${ }^{60} \mathrm{Co}$ doped calibration units. The average PHA value for each PMT is calculated from events from the CAL units with a window on the analog sum constraining the 1.17 and $1.33 \mathrm{MeV}$ gamma rays (see Fig. 15). A change in these averages can be translated into a change of the gains. During the instrument calibration, gain changes of up to $30 \%$ were observed, but the changes over a day were always less than $10 \%$. Similar gain variations have been observed in flight.

The final conversion from PMT output (volts) into energy ( $\mathrm{keV}$ ) was determined using a number of radioactive sources. For each source the relevant peak position in channel numbers was fitted and converted into volts taking into account gain corrections. The $\mathrm{D}_{2}$ energy was calibrated using the photopeak

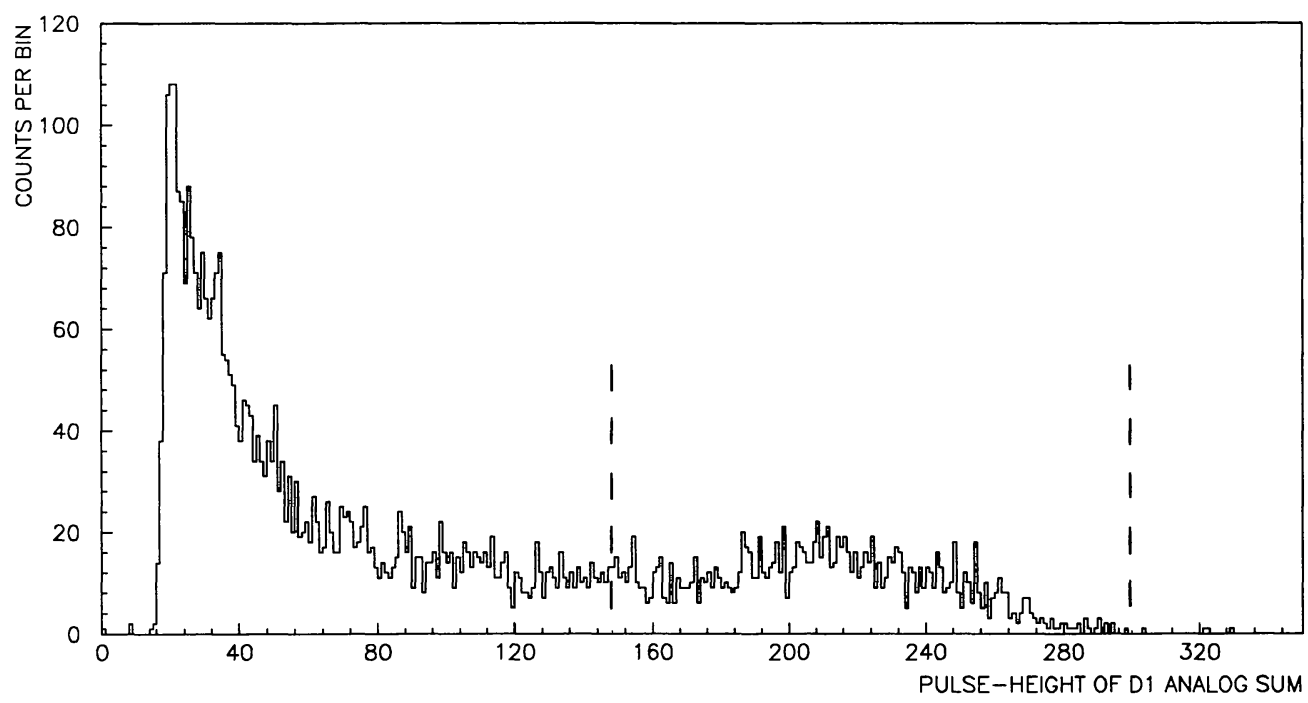

FIG. $15 a$

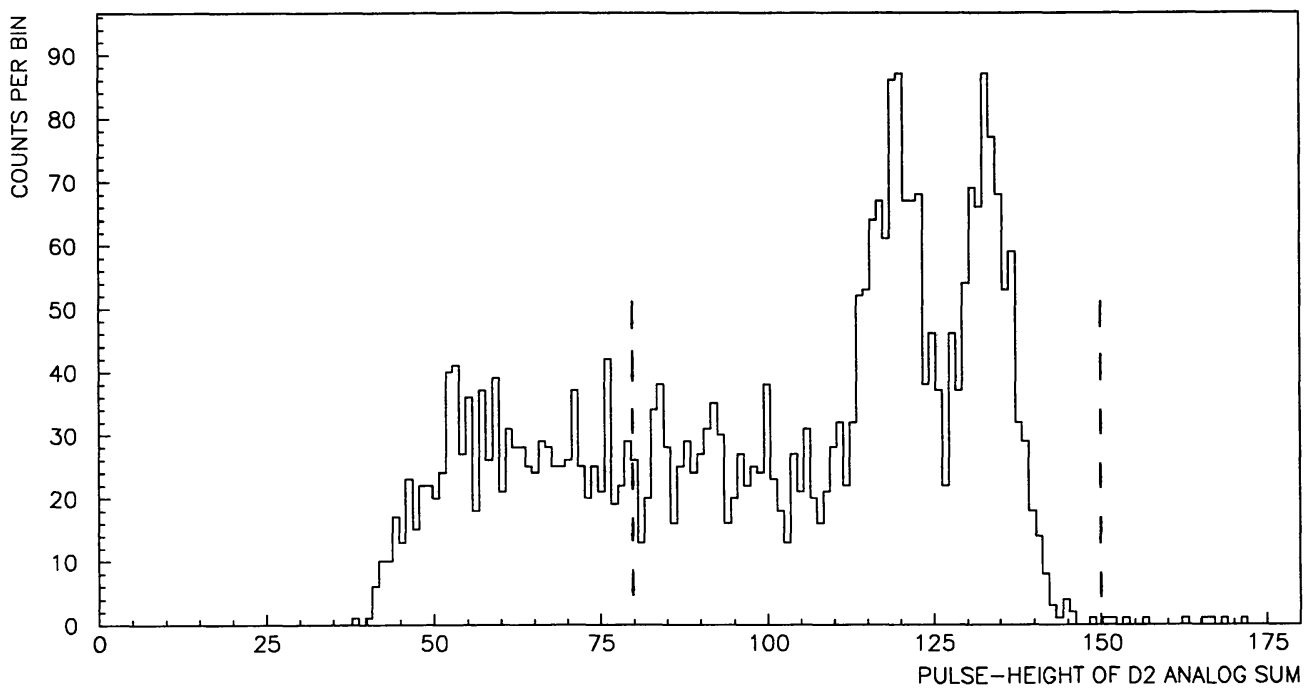

FIG. $15 b$

FIG. $15 .-(a) \mathrm{D}_{1}$ and $(b) \mathrm{D}_{2}$ spectra of ${ }^{60} \mathrm{Co}$ events from the calibration units. In the $\mathrm{D}_{2}$ spectrum the photopeak signature is clearly visible ( 1.17 and 1.33 $\mathrm{MeV}$ ), whereas in the $\mathrm{D}_{1}$ spectrum only the Compton edge is observed. For each PMT the average is determined for events in the indicated calibration-average window. 
signatures ranging from $0.36 \mathrm{MeV}\left({ }^{133} \mathrm{Ba}\right)$ up to $6.131 \mathrm{MeV}$ $[\mathrm{CaF}(p, \gamma)]$. The data were nicely described by a linear function $(\mathrm{rms}=12 \mathrm{keV})$. Energy-corrected spectra at $12.14 \mathrm{MeV}$ $\left[{ }^{11} \mathrm{~B}(p, \gamma)\right]$ and $20.525 \mathrm{MeV}[\mathrm{T}(p, \gamma)]$ were compared with Monte Carlo simulations for these energies, since no distinct photopeak can be seen in these spectra. The measurements and the simulations are in close agreement. The $\mathrm{D}_{1}$ modules were calibrated over a range from $0.20 \mathrm{MeV}\left({ }^{133} \mathrm{Ba}\right)$ to 2.5 $\mathrm{MeV}\left({ }^{24} \mathrm{Na}\right)$. The spectra recorded with liquid scintillators do not exhibit a clear photopeak signature; therefore, the backscatter measurement illustrated in Figure 14 was used. In this measurement an auxiliary detector is placed at a scattering angle of about $180^{\circ}$. A coincidence between this detector and the $\mathrm{D}_{1}$ module is required. Knowing the actual scatter angle, we can calculate the energy deposit in the $D_{1}$ modules (Diehl \& Graser 1981).

A typical $\mathrm{D}_{1}$ backscatter spectrum (for ${ }^{137} \mathrm{Cs}$ ) is shown in Figure 16. The backscatter technique was employed to calibrate COMPTEL using various radioactive calibration sources $\left({ }^{133} \mathrm{Ba},{ }^{22} \mathrm{Na},{ }^{137} \mathrm{Cs},{ }^{54} \mathrm{Mn}\right.$, and $\left.{ }^{24} \mathrm{Na}\right)$. These sources provided a calibration at energies of $339,471,639,1059,1150$, and 2517 $\mathrm{keV}$. (Limitations imposed by the mechanical support of COMPTEL during the calibration period prevented a proper backscatter calibration at higher energies using photons produced by the Van de Graaff accelerator.)

There are additional data available which allow us to verify the energy calibration and gain corrections. Most of these will be applied in flight, but a number of them have been applied for the calibration measurements as well: long-term gain variations are corrected for using triple coincidences between a calibration unit, $\mathrm{D}_{1}$ module, and a $\mathrm{D}_{2}$ module. In addition, onboard spectra for events from the calibration units are accumulated which help in assessing the differences in the calibration averages of the calibration measurements (see Fig. 15) and the in-flight situation due to random coincidences. Background lines (e.g., $2.2 \mathrm{MeV}$, due to neutron capture in the $\mathrm{D}_{1}$ and in the hydrazine tanks of the spacecraft) are used to verify the in-flight energy calibration.

\section{2. $D_{1}$ Module Performance \\ 5.2.1. Spectral Response}

The $\mathrm{D}_{1}$ detectors were designed to maximize the probability of a single Compton interaction. Studies have shown for typical COMPTEL telescope events (with typical event-selection criteria) that less than $3 \%$ of the events involve multiple scattering in a $\mathrm{D}_{1}$ detector. The response function for single Compton-scatter events is relatively simple. It can be well represented by a single Gaussian function. The calibration of the response function can then be given as a function which relates the resolution to the deposited energy. This relation is expected to follow roughly an $E^{0.5}$ functional form (see Fig. 17).

\subsubsection{Event-Location Characteristics}

The characteristics of the event-location process have been determined by "mapping" each module with a collimated beam of either ${ }^{60} \mathrm{Co}\left(\mathrm{D}_{2}\right)$ or ${ }^{22} \mathrm{Na}\left(\mathrm{D}_{1}\right)$ gamma rays. Approximately 900 different collimator positions were used to map the spatial response of each $\mathrm{D}_{1}$ module. In this way, one can associate a particular event signature (i.e., a particular set of relative PMT signals) with a specific location within the module. The data were subsequently used to derive location maps using a neural-net learning process (Connors et al. 1991). It is these location maps which are routinely used in the location of COMPTEL events in the $D_{1}$ module. The use of neural nets in the location process is a fast and effective means of locating events. This is important given the large data volume during the orbital lifetime of COMPTEL.

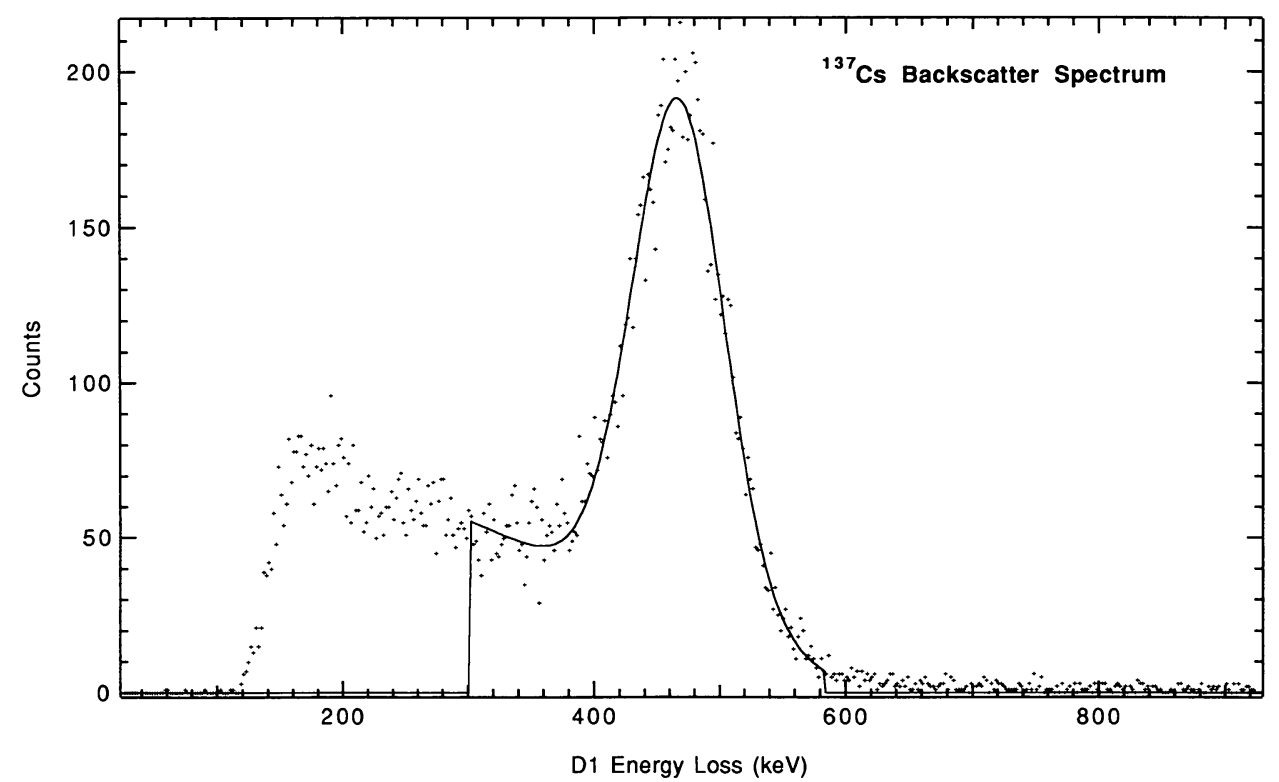

Fig. 16.-Typical backscatter $D_{1}$ spectrum. In this case the $662 \mathrm{keV}$ line from ${ }^{137} \mathrm{Cs}$ produces a backscatter feature at $468 \mathrm{keV}$. The fit to these data is also shown. Note that the true $D_{1}$ response consists only of the Gaussian part of the spectrum; the continuum results from accidental coincidences between the $D_{1}$ detectors and the external gating detector. 


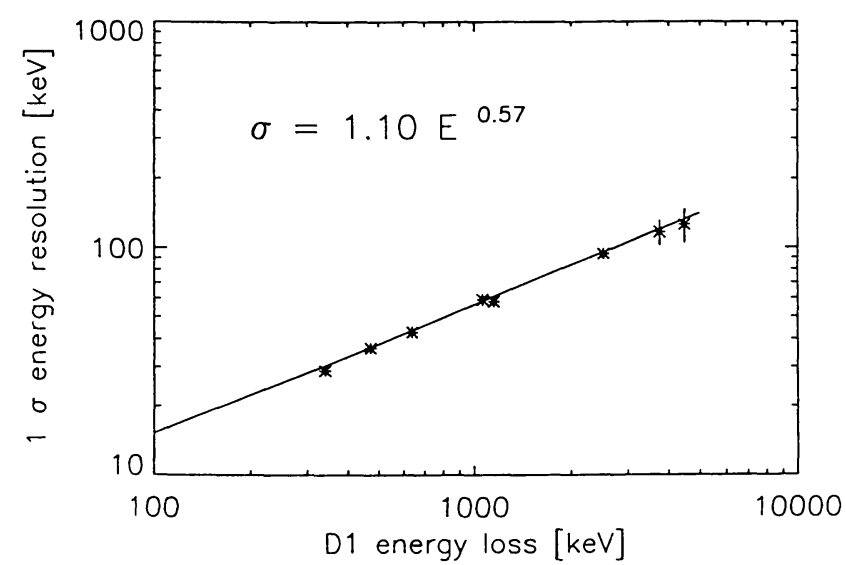

FIG. 17.-The $1 \sigma$ energy resolution of the $\mathrm{D}_{1}$ modules as a function of energy. The measured calibration points are shown, along with the extrapolated fit to those data.

The absolute spatial resolution of the $D_{1}$ modules is measured by comparing the derived event locations for collimated events with the known collimator position. The $1 \sigma$ event location is found to vary from $0.6 \mathrm{~cm}$ (in front of the PMTs) to 6.1 $\mathrm{cm}$ (between the PMTs). The average $1 \sigma$ spatial resolution (averaged over all collimator positions) is $2.3 \mathrm{~cm}$. The $1 \sigma$ event-location map for a single $D_{1}$ module is illustrated in Figure 18.

The uniformity of the event-location process can be studied by analyzing data from a uniformly irradiated module. Such a situation can be achieved, for instance, by using room background data, where one expects a uniform distribution of events within a cell. Alternatively, one can use data obtained from a relatively distant calibration source. These so-called flood maps show evidence for a somewhat nonuniform spatial

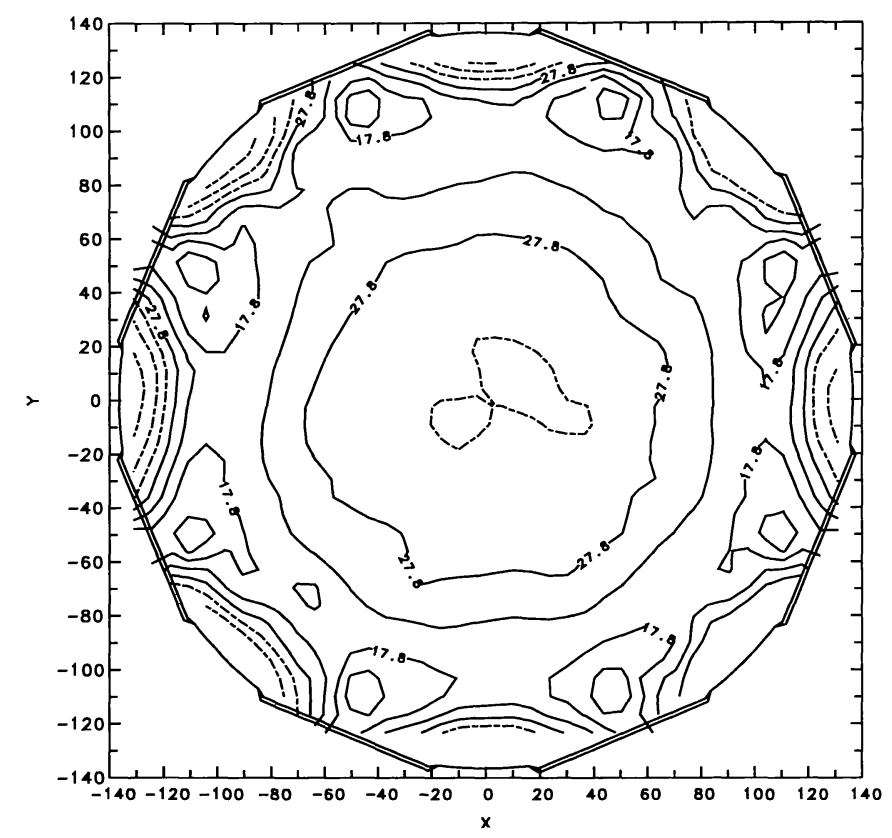

FIG. 18.-A $1 \sigma$ location map for a single $D_{1}$ module. The contour levels are given in millimeters. Coordinates $x$ and $y$ are defined in Fig. 1 .

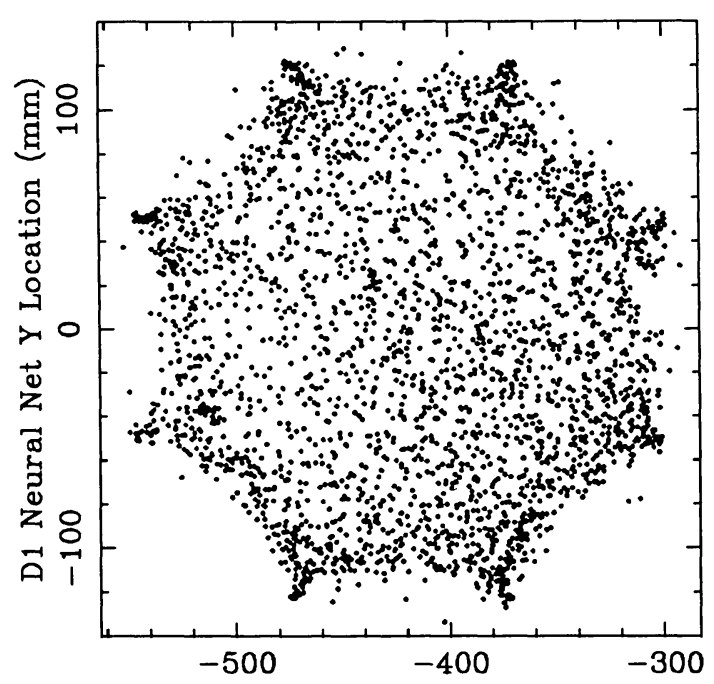

D1 Neural Net X Location ( $\mathrm{mm}$ )

FIG. 19.-Flood map of a $D_{1}$ module. For coordinates see Fig. 1.

distribution of events within the module, as shown in Figure 19.

There is a tendency for events between the PMTs to be "located" in front of the PMTs. This results in an apparent clustering of events in front of each PMT. This behavior is completely consistent with the measured spatial resolution of the cell and does not significantly affect the imaging characteristics of the telescope.

\subsubsection{Pulse-Shape Discrimination}

The capability of the $\mathrm{D}_{1}$ detector to discriminate between gamma-ray- and neutron-induced events is illustrated in the two-dimensional scatter plot of Figure 20. After application of a linearization factor to the actually curved energy/pulseshape curve, gamma rays are found-independently of their

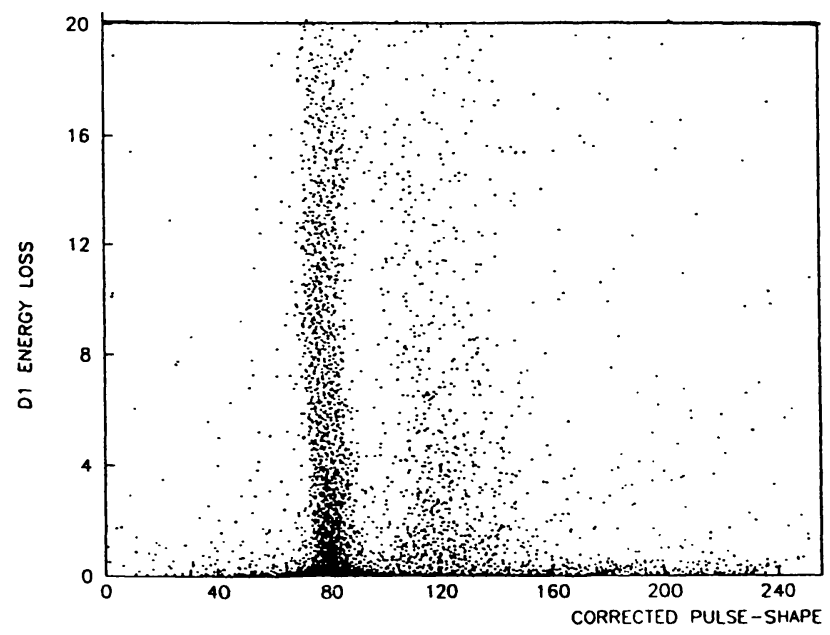

Corrected PSD/E1 scatter plot

FIG. 20.-Separation of gamma-ray- and neutron-induced events in $D_{1}$ by pulse-shape measurements. 
energy loss-around channel 80 , whereas neutron-induced events appear in channels above 100 .

\section{3. $D_{2}$ Module Performance}

During its calibration the $\mathrm{D}_{2}$ detector was homogeneously irradiated by gamma rays produced at low energies by various radioactive isotopes $\left({ }^{137} \mathrm{Cs},{ }^{54} \mathrm{Mn},{ }^{22} \mathrm{Na},{ }^{24} \mathrm{Na}, \mathrm{Am} / \mathrm{Be}\right)$ and at high energies by nuclear reactions $[\mathrm{F}(p, a) \mathrm{O} ; \mathrm{B}(p, \gamma) \mathrm{C} ; \mathrm{T}(p$, $\gamma) \mathrm{He}]$. The data of these calibrations were analyzed by fitting an analytical expression to the measured energy spectra which describes the various physical processes: photoelectric absorption, single and multiple Compton scattering, and pair production. The analytical approximation is based on three Gaussians for the photopeak and the two escape peaks, the Klein-Nishina formula for the Compton distribution, and an energy-independent Compton background, which is due to the material surrounding the $\mathrm{NaI}(\mathrm{Tl})$ crystal of a $\mathrm{D}_{2}$ module. Up to an energy of $12.14 \mathrm{MeV}$, the original Klein-Nishina formula was used; above this energy it was modified with an energy-dependent empirical factor $f(E)$ of the form

$$
f(E)= \begin{cases}\exp \left[-\mu(E) 2.9 \ln \left(E_{\mathrm{MeV}}-11.14\right)\right] & \text { for } E \geq 12.14 \mathrm{MeV}, \\ 1 \quad \text { for } E \leq 12.14 \mathrm{MeV} ; & \end{cases}
$$

$\mu(E)$ is the linear absorption coefficient for $\mathrm{NaI}(\mathrm{Tl})$. Examples of such fits to the data at 4.44 and $20 \mathrm{MeV}$ are shown in Figures 21 and 22, respectively.

From such fits to combined energy spectra of the 14 modules the resolution as a function of energy for the $D_{2}$ detector was obtained ( see Fig. 23). It can be represented by a function of the form

$$
\sigma(E)=10^{-2}\left(9.86 E_{\mathrm{MeV}}+4.143 E_{\mathrm{MeV}}^{2}\right)^{1 / 2} .
$$

The three data points at energies above $10 \mathrm{MeV}$ (Fig. 23) correspond to the width of the distribution at these energies. They are not the photopeak resolutions.

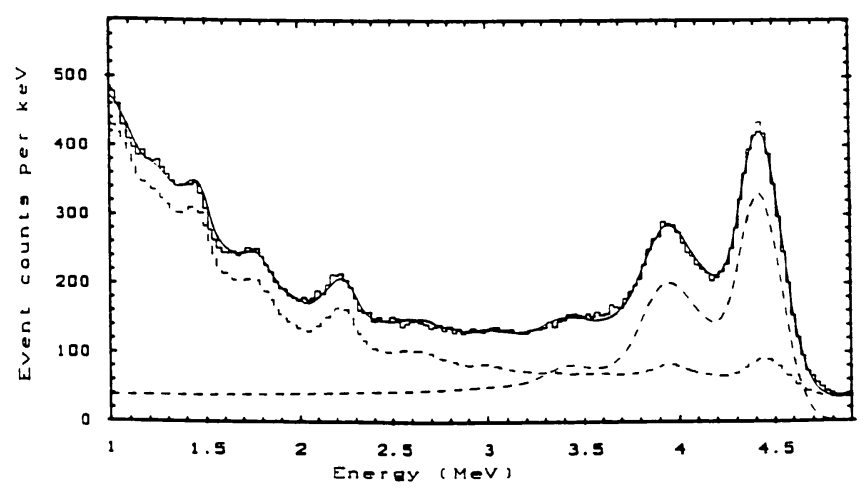

FIG. 21.- $\mathrm{D}_{2}$ energy response for a monoenergetic gamma-ray source $(\mathrm{Am} / \mathrm{Be}$ ) with an energy of $4.438 \mathrm{MeV}$. The data (histogram) and the fit through the data (thick line) are shown for energies above $1 \mathrm{MeV}$. The dashed line shows the contribution of the four components to the response: the photopeak, the first and second escape peaks, and the Compton distribution. The dashed histogram is the background.

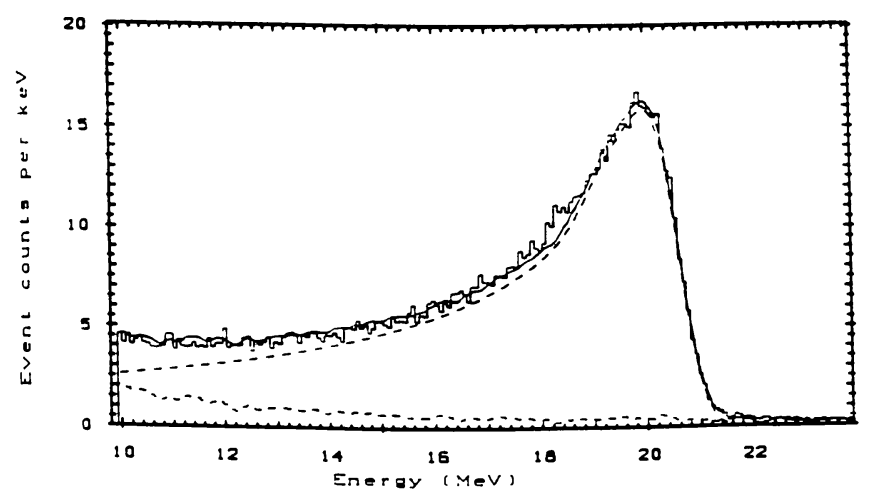

FIG. 22.- $\mathrm{D}_{2}$ energy response for monoenergetic gamma rays (from the target) with an energy of $20.53 \mathrm{MeV}$. The data (histogram) and the fit through the data (thick line) are shown for energies above $10 \mathrm{MeV}$. The modified Klein-Nishina distribution and the background are shown by the dashed lines.

Like $D_{1}$, the $D_{2}$ detector was also mapped with a collimated radioactive source $\left({ }^{22} \mathrm{Na}\right)$ which emits simultaneous gamma rays at $511 \mathrm{keV}$ and $1.27 \mathrm{MeV}$. The collimator was placed at 686 positions above each module, and at each position about 2000 gamma-ray events were collected. Again, a neural-net algorithm was used to reconstruct the interaction location for each event. With this approach it is possible to locate an event to an accuracy of $1.5 \mathrm{~cm}(1 \sigma$ value). However, the event-location capability is not the same across the $\mathrm{D}_{2}$ module. There exist regions where the events are located less well $(2.5 \mathrm{~cm})$ and regions where they are located better $(0.5 \mathrm{~cm})$. The $1 \sigma$ event-location contour map for a single $\mathrm{D}_{2}$ detector module is illustrated in Figure 24.

The energy independence of the maps was proved by mapping one module at $6.1 \mathrm{MeV}$ and at $20.5 \mathrm{MeV}$ (Böhm 1984). The maps obtained at those energies compared well with the maps at an energy of $1.27 \mathrm{MeV}$ which are used in the neural-net algorithm.

Therefore, one $\mathrm{D}_{2}$ map is used over the entire energy range.

\subsection{Time-of-Flight Measurements}

The time of flight (TOF) between the $\mathrm{D}_{1}$ detector and the $\mathrm{D}_{2}$ detector is measured with an accuracy of $0.25 \mathrm{~ns}$. A typical

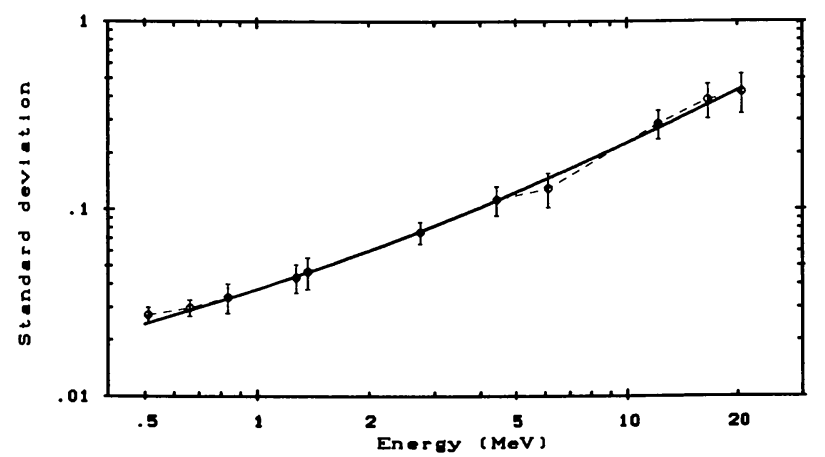

FIG. 23.-The $1 \sigma$ energy resolution of the $\mathrm{D}_{2}$ modules as a function of energy. 


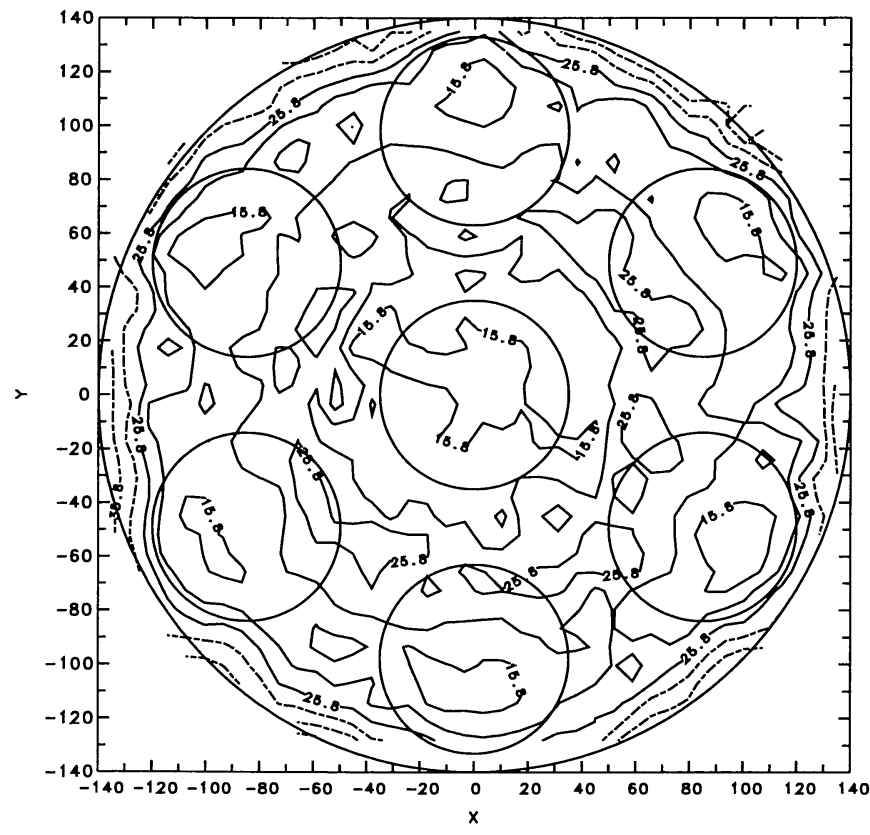

FIG. 24.-A $1 \sigma$ event-location map for a single $\mathrm{D}_{2}$ module. The contour levels are given in millimeters. For coordinates see Fig. 1.

TOF spectrum for a single $D_{1}$ detector module with a single $D_{2}$ detector module is shown in Figure 25. The backward-scattered events (first an interaction in the $D_{2}$ and then in the $D_{1}$ detector) and the forward-scattered events can be clearly separated. With a resolution of about $1 \mathrm{~ns}(1 \sigma)$ the forward- and backward-scattered TOF peaks are separated by about 11 standard deviations. Close to the energy thresholds of the detectors there is a dependence of the TOF peak position on energy. This effect, which is less than $1 \mathrm{~ns}$ in the $\mathrm{D}_{1}$ detector and less than 3

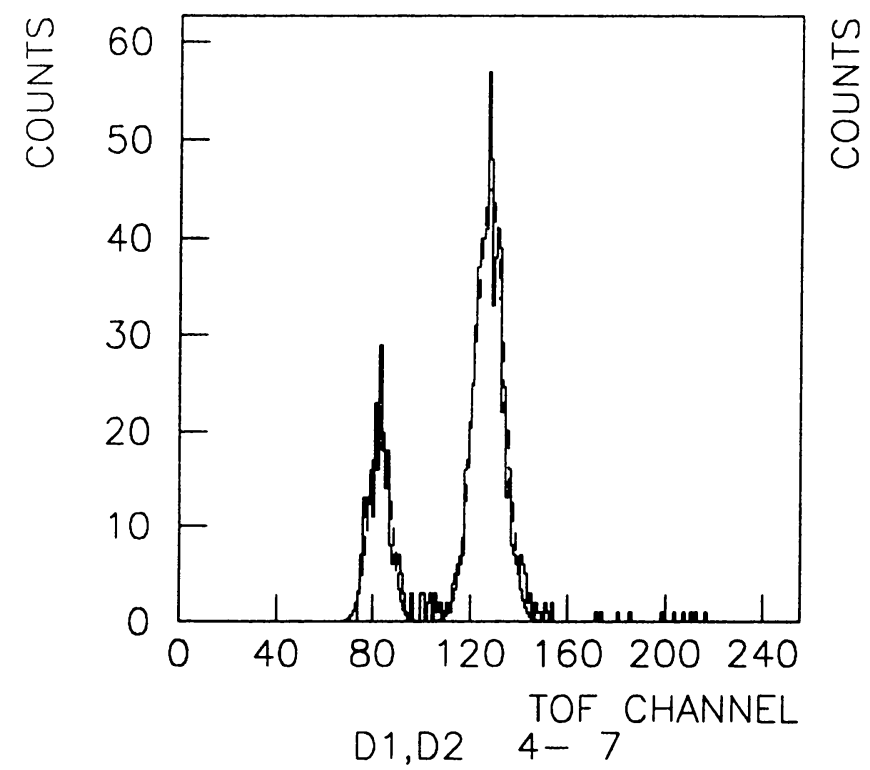

FIG. 25.-Typical TOF spectrum of one $\mathrm{D}_{1}$ detector module with one $D_{2}$ detector module $\left(D_{1}-4, D_{2}-7\right)$. One TOF channel corresponds to 0.25 ns. ns in the $\mathrm{D}_{2}$ detector, is taken into account. The high-energy neutrons, which will show up in the forward-scattered TOF peak, can be effectively rejected using the pulse-shape discrimination (PSD) information from the $\mathrm{D}_{1}$ modules.

\subsection{Veto-Dome Performance}

The task of the anticoincidence subsystem is to reject charged particles entering the telescope with a high probability. Given the charged-particle background of the near-Earth environment, each veto dome has to have a rejection probability which should be larger than $99.99 \%$. A second, less stringent requirement was that the rejection probability per dome should remain better than $99.9 \%$ with only two-thirds of the PMTs working.

The efficiency of each veto dome in rejecting charged particles was tested on ground using atmospheric muons as test particles. For this measurement COMPTEL was configured in the telescope mode with the veto-dome override option on (i.e., the anticoincidence was disregarded, and those events were also recorded which may have triggered a veto dome). Muons were characterized by high-energy deposits in $D_{1}$ (about $15 \mathrm{MeV}$ ) and in $\mathrm{D}_{2}$ (about $45 \mathrm{MeV}$ ), a trigger signal in at least three of the four veto domes, and were selected accordingly during data analysis. To obtain statistically significant results, more than 50,000 muons were collected for each measurement. The result of the data analysis for the nominal thresholds (threshold level 4) is given in Table 8.

The second requirement is met for three veto domes (for $V_{3}$ no such measurement was performed), whereas the first requirement is only met for the veto dome $V_{1}$. But since the rejection probabilities of $V_{2}$ and $V_{3}$ are nearly the same in spite of their different light collection yields, the measurements were probably confused by masquerading events (e.g., by high-energy electrons emitting bremsstrahlung quanta or by pair-production effects), and therefore the true rejection probabilities of the veto domes $\mathrm{V}_{2}, \mathrm{~V}_{3}$, and $\mathrm{V}_{4}$ are in fact better than those given in Table 8.

\section{TELESCOPE PERFORMANCE}

During the $G R O$ mission, COMPTEL can be operated in two different astronomical telescope modes: as a gamma-ray telescope and as a solar neutron telescope. In addition, a burst mode is operated continuously in parallel with the available telescope modes. In this section the properties of COMPTEL as derived from calibration and simulation are described for all three operational modes.

TABLE 8

Minimum Rejection PRobability of Veto Domes

\begin{tabular}{cccc}
\hline \hline & & \multicolumn{2}{c}{ ReJECTION ProbABILITY } \\
\cline { 3 - 4 } VETO & $\begin{array}{c}\text { THRESHOLD } \\
\text { ENERGY } \\
(\mathrm{keV})\end{array}$ & All PMTs & $\begin{array}{c}\text { Two-thirds of } \\
\text { PMTs Only }\end{array}$ \\
\hline DOME & 230 & $>99.99 \%$ & $99.91 \pm 0.02 \%$ \\
$\mathrm{~V}_{1} \ldots \ldots$ & 472 & $99.97 \% \pm 0.01 \%$ & $99.90 \pm 0.02 \%$ \\
$\mathrm{~V}_{2} \ldots \ldots$ & 390 & $99.98 \% \pm 0.01 \%$ & $\ldots$ \\
$\mathrm{V}_{3} \ldots \ldots$ & 316 & $99.90 \% \pm 0.02 \%$ & $99.93 \pm 0.02 \%$ \\
$\mathrm{~V}_{4} \ldots \ldots$ & & &
\end{tabular}




\subsection{The Imaging Gamma-Ray Telescope}

The response of COMPTEL to a beam of monoenergetic photons from a specific incidence direction can be described in a simple way by its cone-shaped pattern in the three-dimensional data space (see $\S 2$ and especially Fig. 4). Imperfections in the location and energy measurements of the interactions, and especially incomplete absorption of the scattered gamma ray, result in a broadening of the cone surface.

In general terms, the response $R$ (the event density in the data space) can be described by

$$
R=R\left(E_{\mathrm{tot}}, \bar{\varphi}, \chi, \psi / E_{\gamma}, \chi_{0} \psi_{0}\right)
$$

A good approximation to this general formula, which preserves all the essential features, is given by

$$
\begin{aligned}
n(\chi, \psi, \bar{\varphi}) & =g(\chi, \psi) \iint_{\chi_{0}, \psi_{0}} I\left(\chi_{0}, \psi_{0}\right) \\
& \times A\left(\chi_{0}, \psi_{0}\right) f\left(\varphi_{g}\left(\chi, \psi, \chi_{0}, \psi_{0}\right), \bar{\varphi}\right) d \chi_{0} d \psi_{0} d \Omega t,
\end{aligned}
$$

where

$$
\begin{aligned}
n(\chi, \psi, \bar{\varphi})= & \text { expected number of counts in a data- } \\
& \text { space cell. } \\
g(\chi, \psi)= & \text { probability that a photon scattered in } \\
& \mathrm{D}_{1} \text { in a direction }(\chi, \psi) \text { encounters a } \\
& \mathrm{D}_{2} \text { module (a purely geometrical ef- } \\
& \text { fect which reflects the fact that the } \mathrm{D}_{1} \\
& \text { and } \mathrm{D}_{2} \text { layers consist of discrete mod- } \\
& \text { ules). } \\
I\left(\chi_{0}, \psi_{0}\right)= & \text { sky intensity distribution. } \\
A\left(\chi_{0}, \psi_{0}\right)= & \text { area of the exposed } \mathrm{D}_{1} \text { surface (about } \\
& 4200 \text { cm }{ }^{2} \text { for normal incidence). } \\
f\left(\varphi_{g}\left(\chi, \psi, \chi_{0}, \psi_{0}\right), \bar{\varphi}\right)= & \text { probability that a photon encounter- } \\
& \text { ing } \mathrm{D}_{1} \text { makes an interaction in both } \\
& \mathrm{D}_{1} \text { and } \mathrm{D}_{2}, \text { scatters through an angle } \\
& \varphi_{g}, \text { and has a measured Compton } \\
& \text { scatter angle of } \bar{\varphi} \text {. It is defined for an } \\
& \text { "infinite" extent of the } \mathrm{D}_{2} \text { layer, } \\
& \text { since the discrete nature of } \mathrm{D}_{2} \text { is in- } \\
& \text { cluded in } g . \text { It includes among other } \\
& \text { things the energy-dependent interac- } \\
& \text { tion proability in } \mathrm{D}_{1} \text { (not therefore } \\
& \text { included in } A) \text { and the effects of in- } \\
& \text { complete absorption in } \mathrm{D}_{2} ; f\left(\varphi_{g}, \bar{\varphi}\right) \\
& \text { is referred to here as the point-spread } \\
& \text { function, since it has the property of } \\
& \text { being (almost) independent of the } \\
& \text { input direction of the photon and } \\
& \text { here is similar to the normal type of } \\
& \text { PSF encountered in optics. } \\
d \Omega= & \text { solid angle covered by the bin at ( } x, \\
& \psi \text { ). } \\
t= & \text { exposure time (in seconds). } \\
&
\end{aligned}
$$

Note that the PSF would be a perfect cone defined by $\varphi_{g}=\bar{\varphi}$ if the energy and position determinations in $D_{1}$ and $D_{2}$ were exact. The finite energy and position resolution lead to a broadening of the cone surface; in particular, there is a filling of regions with $\bar{\varphi}>\varphi_{g}$ due to incomplete absorption in $\mathrm{D}_{2}$ (see $\S 2$ : COMPTEL response). But also other types of nonideal events exist: e.g., multiple scattering in $\mathrm{D}_{1}$, non-Compton interactions in $D_{1}$, incomplete energy loss in $D_{1}$. The latter leads to events which fall outside the ideal response cone.

The response has been determined in three different ways (as described in $\S 4$ ):

1. From calibration measurements of the entire instrument with known monochromatic photon sources at specified positions within the instrument field of view.

2. From calibration measurements of instrument detector modules with known monochromatic photon sources, and composition of these detector module responses to a telescope response via (assumed) physical characteristics of the telescope.

3. From simulation of the physical processes in the detector modules that are experienced by the interacting photons.

All three response-determination methods have their distinct advantages and disadvantages, and they are compared in the following paragraphs.

Direct calibration.-The response calibration for the telescope as a whole seems to be the straightforward measurement. In reality, however, calibration measurements suffer from imperfections, which to a certain extent can degrade the accuracy of the response determination. Examples of such imperfections are the following:

1. Nonmonochromatic photon sources, the contribution of which cannot be accurately assessed. Background subtractions are affected by the background measurement statistics and sometimes yield "negative counts" in some bins.

2. Calibration photon source position not characteristic for the photon source position during the astronomical observation (e.g., not at virtually infinite distance).

3. Calibration photon sources available at a few energies only.

Calibration of telescope components.-The response determination from calibrations of telescope components avoids the direct impact of calibration inadequacies on response inaccuracies-however, at the price of involving some prior (maybe imperfect) knowledge about how the response depends on the properties of the single components. In this approach it is assumed that the incoming photon suffers one single Compton scattering process in the upper detector and that the scattered gamma ray interacts with the lower detector. For a given input energy of the photon and a given scatter angle, the response of the telescope is then mainly determined by the characteristics of the individual scintillation detectors, namely, their energy and spatial resolution. This response is called the "semiempirical" or "model" response.

Simulations. - The response determination by simulation is not restricted to a few energies but can be performed at any energy. The results from simulations can be compared with those obtained from calibration. If the results of both methods are consistent at the calibration energies, then one can be confident that the simulation also provides a proper description of the telescope at other energies. 

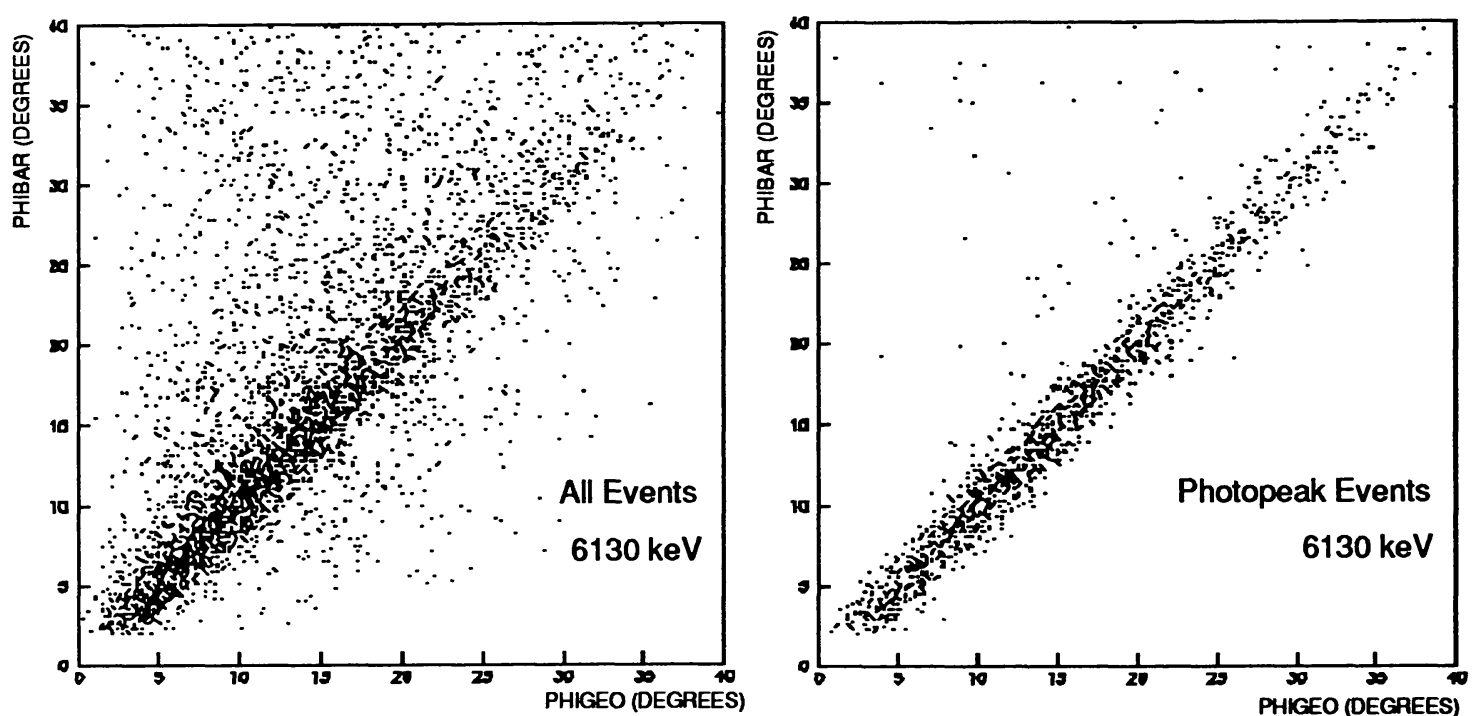

FIG. 26. - Illustration of the conelike structure of the telescope response in the three-dimensional data space. The simulated gamma-ray source (6.1 $\mathrm{MeV}$ ) was on-axis. Left: all events accepted; right: photopeak events accepted. The cone is obtained by rotating the $45^{\circ}$ diagonal around the $\bar{\varphi}$-axis.

\subsubsection{Spatial Response of COMPTEL (Point Spread Function)}

The spatial (imaging) response of COMPTEL has been derived in terms of a distribution in three-dimensional dataspace coordinates $(\chi, \psi, \bar{\varphi})$ for photons of specific energy and incidence angles. The response is also defined as a function of measured energy deposit.

For illustration, Figure 26 shows the cone-shaped structure in the data space from a simulated 6.1 MeV on-axis gammaray source. The angle $\varphi_{\text {geo }}$ plotted on the abscissa is the true scattering angle, known from the position of the source. The totally absorbed events along the $45^{\circ}$ cone (right-hand side of Fig. 26) are clearly visible, as well as the events, not totally absorbed in $\mathrm{D}_{2}$, which fill the inner part of the cone (left-hand side of Fig. 26). Also, events outside the cone are clearly visible (left-hand side of Fig. 26). Most of them had an incomplete energy loss in $D_{1}$.

The width of the spatial response ( the effective angular resolution) can be illustrated in a simple way as the distribution which represents, for each event, the difference between the true geometrical scatter angle $\left(\varphi_{\text {geo }}\right)$ and the scatter angle derived directly from the measured event parameters $(\bar{\varphi})$. Such "angular resolution measure" (ARM) spectra are displayed in Figure 27 for the three response determination methods. We expect that all events from a gamma-ray point source will be concentrated around $\bar{\varphi}-\varphi_{\text {geo }}=0$. Two conclusions can be derived from Figure 27. First, the ARM spectra show the main peak due to fully absorbed events at $\bar{\varphi}-\varphi_{\mathrm{geo}}=0$ and an asymmetric tail shifted toward larger $\bar{\varphi}-\varphi_{\mathrm{geo}}$ values, reflecting the partially absorbed events. Second, the three different methods give results which are very similar. The widths of the three distributions are practically identical for the three methods. Only in the tails toward positive and negative $\bar{\varphi}-\varphi_{\text {peo }}$ values are there small differences. Because of their availability at all energies, and because of their satisfactory agreement with the calibration results, the simulated and modeled point spread functions are used in practice for the COMPTEL image reconstruction.
The width of the angular resolution measure depends only weakly on the gamma-ray energy (seen from Fig. 28, which displays the $1 \sigma$ width as a function of the gamma-ray energy). Above $2 \mathrm{MeV}$ the width is mainly determined by the location uncertainties in the two detectors, while at lower energies the energy resolution within both detectors dominates. The results of Figure 28 are for a source at finite distance $(\approx 9 \mathrm{~m})$. One would expect the angular resolution to improve for sources at indefinite distance.

\subsubsection{Energy Response of COMPTEL}

The spectral response (energy response) of the telescope was measured for the gamma-ray energies and angles of incidence
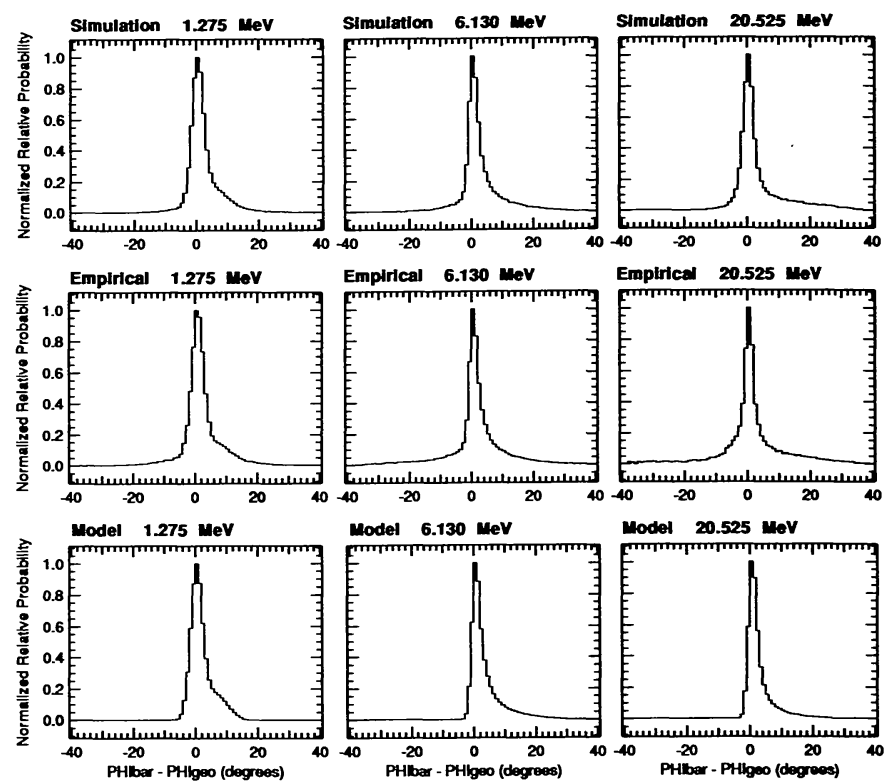

FIG. 27.-ARM spectra at three different gamma-ray energies (1.27, 6.1, and $20.5 \mathrm{MeV}$ ) obtained by using three different methods: calibration, a semiempirical model, and simulation. 


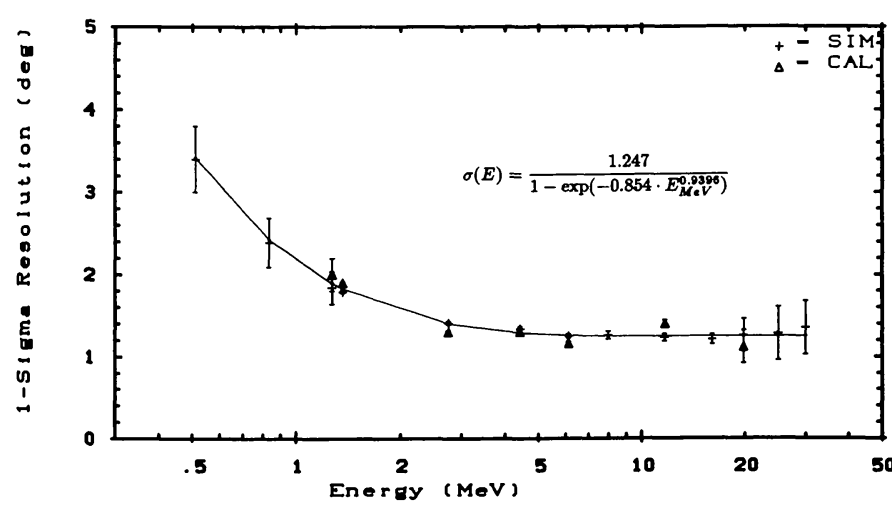

FIG. 28.-Telescope angular resolution (as $1 \sigma$ width of ARM spectra) as determined from calibrations and simulations.

of Table 6. Examples of the energy response of the full telescope are illustrated in Figure 29 for a radioactive source of ${ }^{24} \mathrm{Na}$ ( 1.37 and $2.75 \mathrm{MeV}$ ) and for a boron target $(4.43,12.14$, and $16.57 \mathrm{MeV}$ ) (on-axis incidence in both cases).

The measured energy spectra are characterized by a full-energy peak and a tail toward lower measured energy deposits due to incomplete absorption of the scattered photons in the lower detector plane. The full-energy peak is clearly visible up
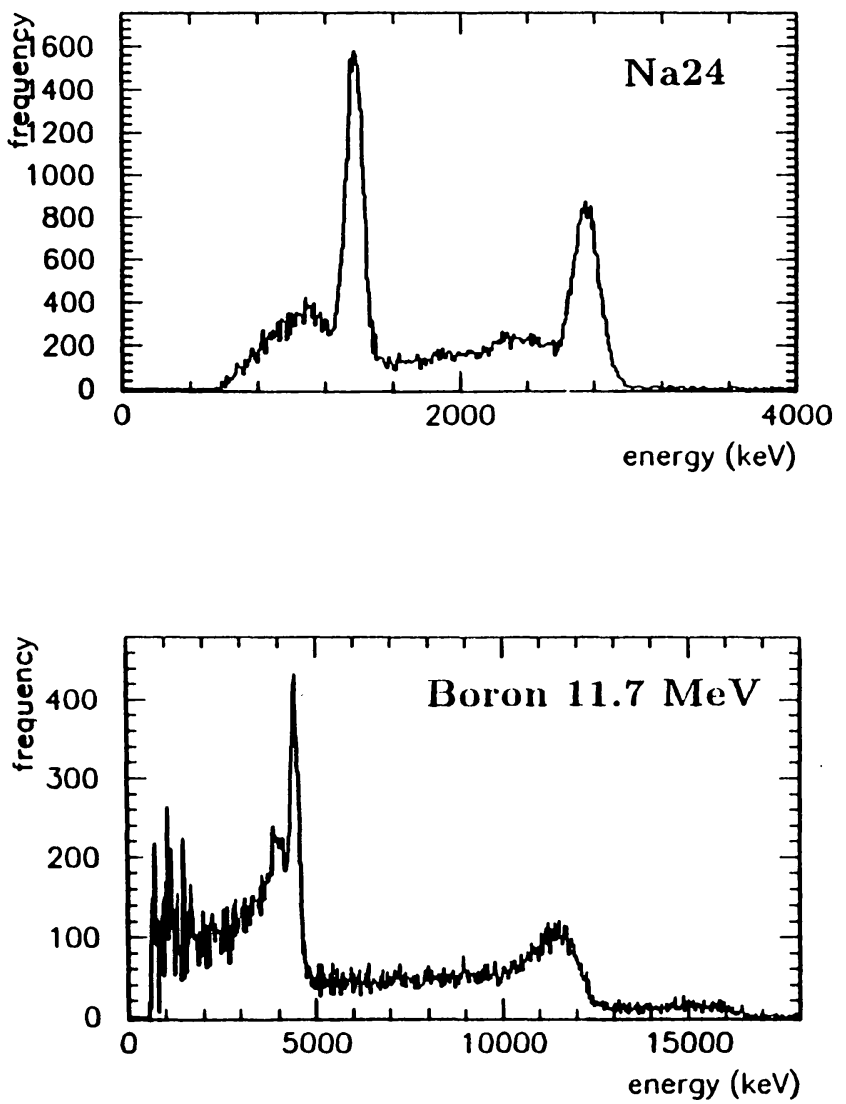

FIG. 29.-Energy-response measurements for the telescope with calibration photons from radioactive ${ }^{24} \mathrm{Na}(1.37$ and $2.75 \mathrm{MeV})$ and from the boron target $(16.57 \mathrm{MeV}$ and $12.14,4.43 \mathrm{MeV})$. (background is subtracted.) to about $10 \mathrm{MeV}$. At higher energies the superposition of photopeak, escape peaks, and Compton tail in $\mathrm{D}_{2}$ determine the response signature of the telescope.

Figure 30 summarizes the energy resolution characteristics as determined from the width of the full-energy peak features, based on calibration measurements and on Monte Carlo simulations. From 1 to $6 \mathrm{MeV}$ the resolution improves from about $10 \%$ FWHM to $5 \%$ FWHM.

\subsubsection{Effective Detection Area}

The absolute COMPTEL detection area at different angles of incidence were derived for a set of nominal event-selection parameters ( somewhat different from the values to be applied to the actual flight data). These are the following:

1. $\mathrm{D}_{1}$ energy threshold: $50 \mathrm{keV}$.

2. $\mathrm{D}_{2}$ energy threshold: $500 \mathrm{keV}$.

3. Time-of-flight window: channels 110-255 (see Fig. 25).

4. Pulse-shape window: channels 0-100 (see Fig. 20).

5. No signal in any of the four veto domes (energy thresholds of veto domes: $1.35 \mathrm{MeV}$ for $\mathrm{V}_{1}, 1.14 \mathrm{MeV}$ for $\mathrm{V}_{2}, 1.2$ $\mathrm{MeV}$ for $\mathrm{V}_{3}$, and $0.95 \mathrm{MeV}$ for $\mathrm{V}_{4}$ ).

These nominal selections can be supplemented by secondary selections, namely, selection in derived scatter angle $\bar{\varphi}$ (for specific background suppression), selection in measured energy deposits (for analysis of specific gamma-ray lines), and selection of specific "event circles" (for specific point-source analysis). The detection area is defined by

$$
A_{\text {eff }}(\vartheta)=\frac{N_{\text {acc }}}{\phi_{\text {exp }} f_{\text {air }}}
$$

where $N_{\text {acc }}$ is the number of events accepted within a given time interval, $\phi_{\exp }$ is the fluence (in $\mathrm{cm}^{-2}$ ) of gamma rays exposed to the telescope within the same time interval, and $f_{\text {air }}$ is a reduction factor, which accounts for the absorption of the gamma rays in air; $A_{\text {eff }}(\vartheta)$ is the effective projected area of COMPTEL perpendicular to the beam direction of gamma rays. The fluence $\phi_{\exp }$ was calculated either from the radioactive source strengths or measurements of the nuclear reaction gamma-ray beam by means of a calibrated beam monitor.

Figure 31 shows the effective detection area for vertical incidence $\left(\vartheta=0^{\circ}\right)$ as a function of energy.

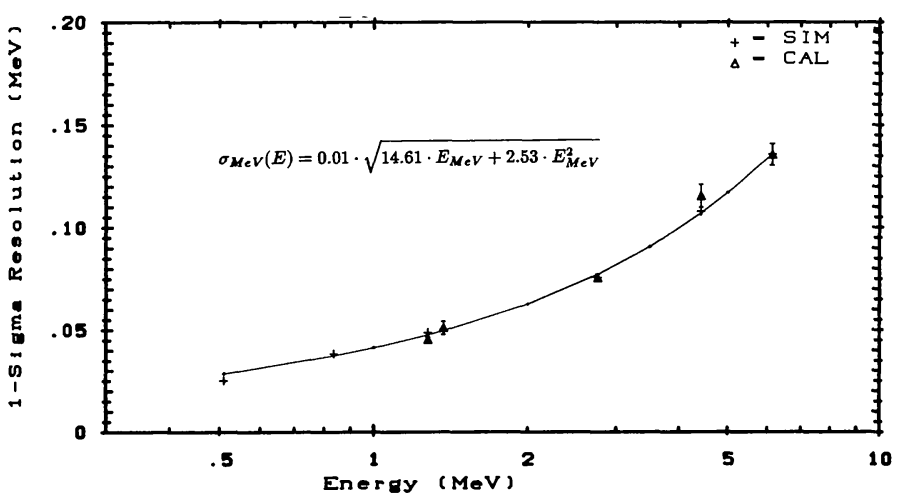

FIG. 30.-Telescope full-energy peak resolution summary (as determined from calibrations and simulations). 


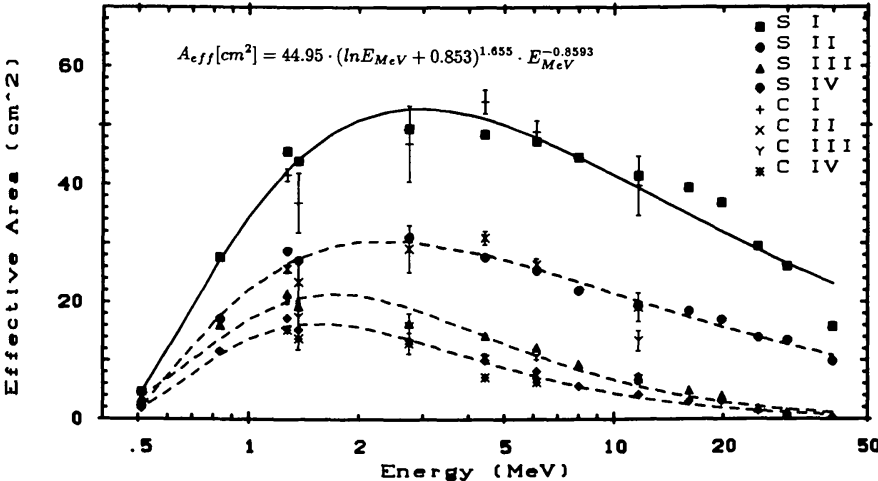

FIG. 31.-Effective detection area determined from calibration and simulation at normal incidence and at a number of different energies. The symbols in the figure have the following meanings: S: data points from simulation; C: data points from calibration; I: no event selections applied; II: event selection according to "extreme" $\bar{\varphi}$ distribution, defined in $\S 2$; III: event selection on $3 \sigma$-wide full-energy peak; IV: event selection on 3 $\sigma$-wide ARM window around $\varphi_{\text {geo }}-\bar{\varphi}=0$. An analytical fit to the top curve (no event selection applied) is given in the figure.

Four cases are considered:

1. No secondary selections were applied.

2. The extreme $\bar{\varphi}$ restriction (defined in $\S 2$ ) was applied to achieve an energy-dependent suppression of the background.

3. A selection of measured energies was applied to accept only events from a gamma-ray line within its $3 \sigma$-wide full-energy peak.

4. A selection on event circles was applied: only those events were accepted which fall into the $3 \sigma$-wide ARM window around $\varphi_{\text {geo }}-\bar{\varphi}=0$.

The error bars on the calibration data points include systematic uncertainties of the radioactive source and beam strength, and of the background subtraction. The statistical uncertainties are negligible.

The dependence of the effective detection area on the angle of incidence $\vartheta$ defines the field of view of the telescope. As described in $\S 2$, the field of view can be influenced by the energy thresholds in $D_{1}$ and $D_{2}$ and by $\bar{\varphi}$ restrictions. Figure 32
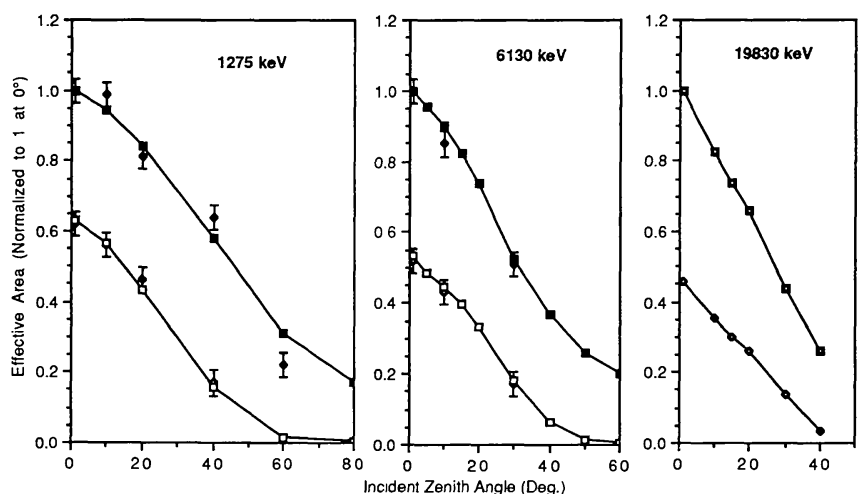

FIG. 32.-COMPTEL field of view as derived from calibration and simulation energies and for two different sets of event selections: top curve: all events above the detector thresholds; bottom curve: events above the detector thresholds satisfying the extreme $\bar{\varphi}$ restrictions. Results for each energy are normalized to unity at $0^{\circ}$ for the least restrictive selections. displays the dependence of the effective detection area with off-axis angle $\vartheta$ at energies of $1.27,6.1$, and $19.830 \mathrm{MeV}$. Two different event selections were considered: no secondary selections and the "extreme" $\bar{\varphi}$ restriction. Monte Carlo results are marked by the lines, and the data points having error bars are the calibration results.

The widest field of view is obtained if no secondary selections are applied to the data. A narrower field of view-at the cost of a lower detection area-is achieved by applying additional $\bar{\varphi}$ event selections. A limitation of the field of view is necessary to reduce the contribution of gamma rays from the Earth's atmosphere.

\subsubsection{Response to Polarized Radiation}

In the response function to polarized radiation the cone mantle function depends on one additional parameter, namely, $\eta$, the angle between the plane of polarization of the incident photon and the scatter plane. For polarized radiation, the function $f\left(\varphi_{\text {geo }}\left(\chi, \psi, \varphi_{0}, \psi_{0}, \bar{\varphi}\right)\right)$ of equation (4) has to be replaced by $f\left(\varphi_{\text {geo }}\left(\chi, \psi, \varphi_{0}, \psi_{0}, \bar{\varphi}\right)\right) r\left(\varphi_{\text {geo }}, \eta+\eta_{0}\right)$, where $r\left(\varphi_{\text {geo }}, \eta\right)$ is the ratio of the scatter probabilities (per unit solid angle) between polarized and nonpolarized gamma rays; $\eta_{0}$ describes the plane of polarization of the incident radiation in the coordinate system of the data space. The differential cross section for polarized radiation is given by (Heitler 1954)

$$
\begin{array}{r}
\frac{d \sigma}{d \Omega}=\frac{r_{e}^{2}}{2}\left\{\frac{1}{1+2 k \sin ^{2}\left(\varphi_{\mathrm{geo}} / 2\right)}+\frac{1}{\left[1+2 k \sin ^{2}\left(\varphi_{\mathrm{geo}} / 2\right)\right]^{3}}\right. \\
\left.-\frac{2 \sin ^{2} \varphi_{\mathrm{geo}} \cos ^{2} \eta}{\left[1+2 k \sin ^{2}\left(\varphi_{\mathrm{geo}} / 2\right)\right]^{2}}\right\},
\end{array}
$$

where $k=E_{\gamma} / m_{0} c^{2}$. Three conclusions can be derived from equation (6): first, at $\eta=0^{\circ}$ the polarization effect is minimal, and at $\eta=90^{\circ}$ it is maximal; second, the polarization effect is more pronounced at large $\varphi_{\text {geo }}$ values; third, the polarization effect decreases with increasing gamma-ray energy.

In Figure 33 the azimuthal $\eta$-dependence of the source cone for $100 \%$ polarized radiation is illustrated for different gammaray energies. The source was assumed to be monoenergetic and on-axis. The integral in data space was taken over the range $20^{\circ}<\varphi_{\text {geo }}<40^{\circ}$. The integral over the data space was normal-

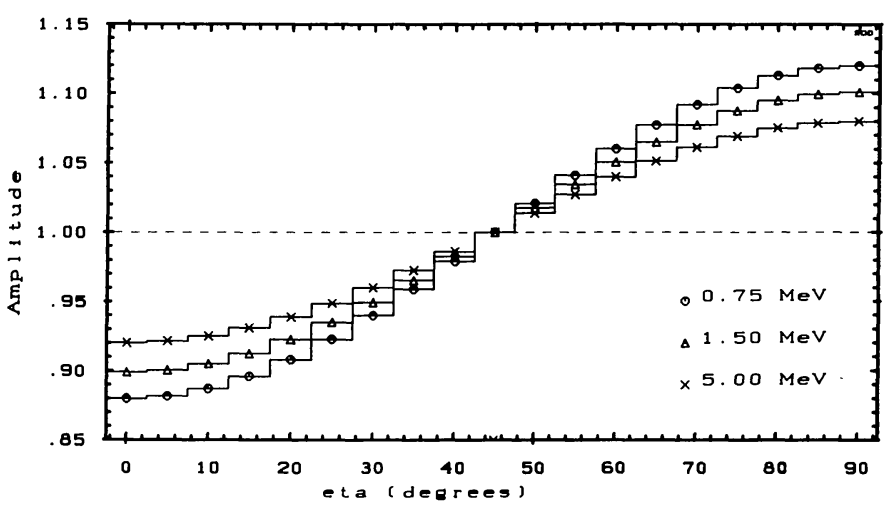

FIG. 33.-Azimuthal dependence of the source cone for $100 \%$ polarized radiation from an on-axis source. Integration along the source cone was performed over the range $20^{\circ}<\varphi_{\text {geo }}<40^{\circ}$. 
ized to 1 at $\eta=45^{\circ}$. At $\eta=0^{\circ}$ and $\eta=90^{\circ}$ the value of the integral deviates by $8 \%$ at $5 \mathrm{MeV}, 10 \%$ at $1.5 \mathrm{MeV}$, and $12 \%$ at $0.75 \mathrm{MeV}$ from the mean value.

This dependence was derived analytically. COMPTEL was never exposed to a beam of polarized gamma radiation during calibration.

\subsubsection{Timing Analysis}

Two of the prime goals of COMPTEL are to study gammaray pulsars and X-ray binary sources. Only the Crab and Vela pulsars had been detected as gamma-ray pulsars, while no detection of $\mathrm{X}$-ray binaries had been made in the COMPTEL energy range prior to the launch of $G R O$.

The tools which permit such a search are provided by the on-board time tagging in combination with analysis software. Gamma-ray events are time-tagged with an accuracy of $125 \mu \mathrm{s}$. Given that the fastest pulsar so far detected has a period of 1.6 $\mathrm{ms}$, the intrinsic time resolution is sufficient to bin those data, provided that the resolution can be maintained during eventtime transformation to an inertial frame of reference. The pulsar time standard refers to the arrival time of the pulses at the solar system barycenter (SSB). Transformation of the spacecraft time to the SSB makes use of the position of the spacecraft. Each event has an associated spacecraft position with an accuracy of about $30 \mathrm{~km}(3 \sigma)$. This translates to a pulse-phase jitter of around $100 \mu \mathrm{s}$, which is compatible with the UTC time resolution.

The spacecraft arrival times are transformed to the SSB using the DE2000 ephemeris of JPL and GRO common software based upon TEMPO code used for radio pulsar data analysis (Taylor \& Weisberg 1989). A cross check of the COMPTEL system results with those of the other $G R O$ instruments demonstrates that the specific data storage and algorithms introduce errors of the order of merely a few microseconds.

\subsection{Response to Gamma-Ray Bursts}

COMPTEL measures cosmic gamma-ray bursts in its "single-detector mode," accumulating spectra from the two $D_{2}$ modules as described in $\S 3$ of this paper. If a burst occurs in the COMPTEL field of view, its position can be localized using data from the "double-scatter mode." This operating mode and COMPTEL's response to sources within the field of view was described above and can also be applied to bursts. The section below provides details on the "single-detector mode" response.

As described in $\$ 3.6$, the COMPTEL burst detector system was calibrated using radioactive sources and beam targets located at various zenith and azimuth angles of the $G R O$ coordinate system. The purpose of this calibration was to derive a baseline channel-to-energy calibration; to determine deadtime correction coefficients, and to establish the burst-response matrix which is a function of energy and source position. The measurements were performed at instrument level at the GSF in Neuherberg in 1987. These were complemented by measurements at spacecraft level (in 1989 during the GRO radioactive source survey) to study the contribution from additional absorbing and scattering mass elements of the spacecraft structure and other $G R O$ experiments at larger off-axis angles $\left(\theta \geq 45^{\circ}\right.$ elevation $)$.
The sources/targets used for these calibrations are listed in Table 9, and Figure 34 shows all calibration source positions in the $G R O$ coordinate system.

In order to derive dead-time correction coefficients for the burst-detector system, two ${ }^{22} \mathrm{Na}$ sources (719 and $176 \mu \mathrm{Ci}$, respectively) were placed at various distances in order to scan a range of varying source intensities.

\subsubsection{Energy Calibration}

The full-energy peak positions of the source runs performed on-axis were derived using a simple Gaussian fit to the data. The photopeak energies were fitted against the channel positions of the peaks, and nearly linear relationships for both low and high ranges were obtained:

Low range: $\quad E(\mathrm{MeV})=-8.9 \times 10^{-3}$

$$
+9.4 \times 10^{-3} x-1.6 \times 10^{-6} x^{2},
$$

High range: $\quad E(\mathrm{MeV})=-11.8+9.5$

$$
\times 10^{-2} x-3.1 \times 10^{-5} x^{2}
$$

where $x$ is the channel number $(x=0-127$ for the low range and $x=128-255$ for the high range).

\subsubsection{Dead-Time Correction}

The main effect is the "priority selection" within the analog electronics subsystem: high-range events are processed with priority over low-range events. This effect is a function of the event rate ("source intensity") and is significant if the time delay between high-range and low-range events is of the order of $17 \mu \mathrm{s}$. The "priority selection" results in a total energy-integrated event rate of the low-range spectrum which is a function of the high-range total rate, the threshold, and the spectral shape of the source intensity. An analytical approach has been derived to correct both low- and high-range rates, where the observable parameters per range (low, high) are total energyintegrated event rate per spectrum and rates from the low / high event scalers and the AE dead times. The derived parameters are the dead-time corrected count rates for each range.

\subsubsection{Energy Response (Mass Shielding Effects)}

The purpose of conducting two calibration sessions, in 1987 at GSF on instrument level and in 1989 at TRW at GRO level,

TABLE 9

\begin{tabular}{|c|c|c|c|}
\hline Source & $\begin{array}{l}\text { Energy } \\
(\mathrm{MeV})\end{array}$ & $\begin{array}{l}\text { GSF } \\
(1987) \\
(\mathrm{mCi})\end{array}$ & $\begin{array}{c}\text { TRW } \\
(1989) \\
(\mathrm{mCi})\end{array}$ \\
\hline${ }^{133} \mathrm{Ba}$ & 0.356 & 0.88 & 10 \\
\hline${ }^{137} \mathrm{Cs} \ldots \ldots \ldots \ldots$ & 0.662 & 0.96 & 2 \\
\hline${ }^{22} \mathrm{Na} \quad \ldots \ldots \ldots \ldots$ & $0.511 / 1.275$ & 0.72 & 2 \\
\hline${ }^{88} Y \ldots \ldots \ldots \ldots$ & $0.898 / 1.836$ & 0.0002 & \\
\hline${ }^{54} \mathrm{Mn} \ldots \ldots \ldots \ldots$ & 0.835 & 0.040 & 2 \\
\hline${ }^{60} \mathrm{Co} \ldots \ldots \ldots \ldots$ & $1.173 / 1.333$ & & 10 \\
\hline${ }^{24} \mathrm{Na} \quad \ldots \ldots \ldots \ldots$ & $1.368 / 2.754$ & 0.15 & 7 \\
\hline${ }^{241} \mathrm{Am} /{ }^{9} \mathrm{Be} \ldots \ldots$. & 4.438 & 0.21 & $\ldots$ \\
\hline $\mathrm{CaF} \ldots \ldots \ldots \ldots$ & 6.130 & 0.7 & $\ldots$ \\
\hline
\end{tabular}

SOURCES FOR THE CALIBRATION OF THE BURST SYSTEM 


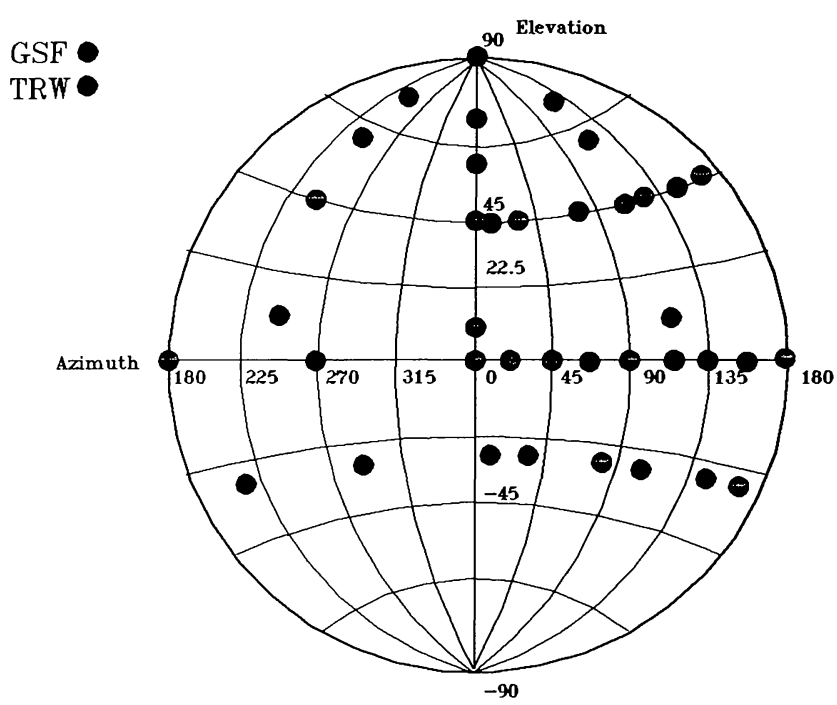

FIG. 34.-Burst calibration: source positions ( $G R O$ coordinate system; see Fig. 1): azimuth $0,+x$-axis; azimuth $90^{\circ},-y$-axis; elevation $+90^{\circ}$ : $+z$-axis.

was to study the contribution of additional mass elements to the recorded burst spectra. This effect is demonstrated in Figure 35, where, both at GSF and at GRO level, a ${ }^{22} \mathrm{Na}$ source of comparable strength and at similar distances was located at the same position (elevation $\Theta=80^{\circ}$, azimuth $A=0^{\circ}$ ). The top panel of Figure 35 displays the source as measured at GSF: the 511 and $1275 \mathrm{keV}$ photopeaks are clearly visible in the lowand high-range spectra, whereas at spacecraft level (Fig. 35, bottom panel) the additional GRO structure, other experiments, and electronic boxes almost completely absorb the two photopeaks. From this result it can be concluded that the response to celestial radiation at this position is almost zero.

Two other mass-distribution effects are clearly visible in Figures 36 and 37: the ${ }^{137} \mathrm{Cs}$ source measurements performed with the $G R O$ setup at constant zenith angle $\boldsymbol{\theta}=45^{\circ}$ and various azimuths are shown in Figure 36. A Gaussian function was fitted to the photopeak data, and the fitted amplitude was normalized to the measurement at $A=8^{\circ}\left(\theta=45^{\circ}\right)$. The azimuthal variation is clearly visible. The minimum absorption occurs at $A \approx 144^{\circ}$.

The effect of mass absorption at constant azimuth is displayed in Figure 37: a ${ }^{133} \mathrm{Ba}$ source was located at various zenith angles at constant azimuth $A=0^{\circ}$. Again, the photopeak distribution was fitted by a Gaussian and the amplitude was normalized to the on-axis value. The most sensitive source location is at $\theta \approx 45^{\circ}$ : the source photons reach the burst detectors by passing between the COMPTEL $D_{1}$ and $D_{2}$ detector arrays with minimum absorption. The response decreases dramatically at zenith distance $\Theta>45^{\circ}$.

For each source at each position, the complete model response function for the D2 detector is fitted to the spectra (gain- and dead-time-corrected and energy-calibrated). Simulations which include the $G R O$ mass model will be used to provide data at energies and source positions in order to provide more response information on a smaller $(E, \Theta, A)$ grid. Finally, interpolation will be applied to derive final in-flight burst-response matrices, required for spectral deconvolution, at approximately $5^{\circ}$ grid size.

\subsection{The COMPTEL Neutron Response}

The performance of COMPTEL as a neutron telescope can be described much the same way as its performance for gamma rays. The response to neutrons is again defined in a three-dimensional scatter space, with the only difference being that the $\bar{\varphi}$-value in the gamma-ray space is replaced by the $\theta_{n}$ value defined in equation (2) in the neutron space.

In the expression for the event counts per data-space cell $n\left(\chi, \psi, \theta_{n}\right)$ (see eq. [4] of $\S 6.1$ ), the point-spread function is no longer defined by the Klein-Nishina cross section, but instead by the $n-p$ scattering process. In addition, the contribution of the D2 detector to the cone blurring is determined not by the energy loss uncertainty in this detector but rather by the inaccuracy of the time-of-flight measurements.

Therefore, in detecting and measuring neutrons from the Sun (or any other point source), the neutrons can be traced backward from $D_{2}$ to $D_{1}$ through the angle $\theta_{n}$ onto a cone mantle restricting the incident direction so as to include the Sun. This geometrical constraint is identical to that of the gamma-ray measurements.

The ionization from a recoiling proton hit by the neutron in the liquid scintillator $\mathrm{NE} 213 \mathrm{~A}(\mathrm{H} / \mathrm{C}$ ratio $=1.333)$ produces a slower pulse in the $D_{1}$ electronics. The rise time (or pulse shape) is measured and used as a discriminator for electron and proton ionizations. The ideal neutron elastic scatter interaction in COMPTEL then has the signature of a proton pulse shape. The pulse shape from recoil protons is sufficient to discriminate against more than $95 \%$ of electron-recoil events greater than about $1 \mathrm{MeV}$, the energy threshold in $\mathrm{D}_{1}$ for neutron detection. This method of detecting and measuring the neutrons is clean, in that a delayed coincident scatter with the correct pulse shape in $D_{1}$ is required, yielding a large signal-tonoise ratio. Inelastic neutron reactions with carbon also occur in the liquid scintillator, particularly at energies greater than about $50 \mathrm{MeV}$. The carbon interactions in $\mathrm{D}_{1}$ often produce gamma rays, deuterons, or alpha particles which can be identified.

Typical production reactions are the following:

$$
\begin{aligned}
& { }^{12} \mathrm{C}(n, n p){ }^{11} \mathrm{~B}, \\
& { }^{12} \mathrm{C}(n, 2 n){ }^{11} \mathrm{C}, \\
& { }^{12} \mathrm{C}(n, \gamma){ }^{12} \mathrm{C}, \\
& { }^{12} \mathrm{C}\left(n, n^{\prime}\right) 3{ }^{4} \mathrm{He}, \\
& { }^{12} \mathrm{C}\left(n, n^{\prime} p^{\prime} \gamma\right){ }^{11} \mathrm{~B} .
\end{aligned}
$$

These reaction channels can be included to further increase the instrument response to neutrons. These interactions, however, are difficult to interpret because the lost energy (nuclear binding energy or escaping gamma rays) results in inaccurate measurement of $E$ and $\Theta_{n}$. In the case of solar-flare neutrons, selected by time, these events can be used to supplement the information obtained from clean elastic scatters. 

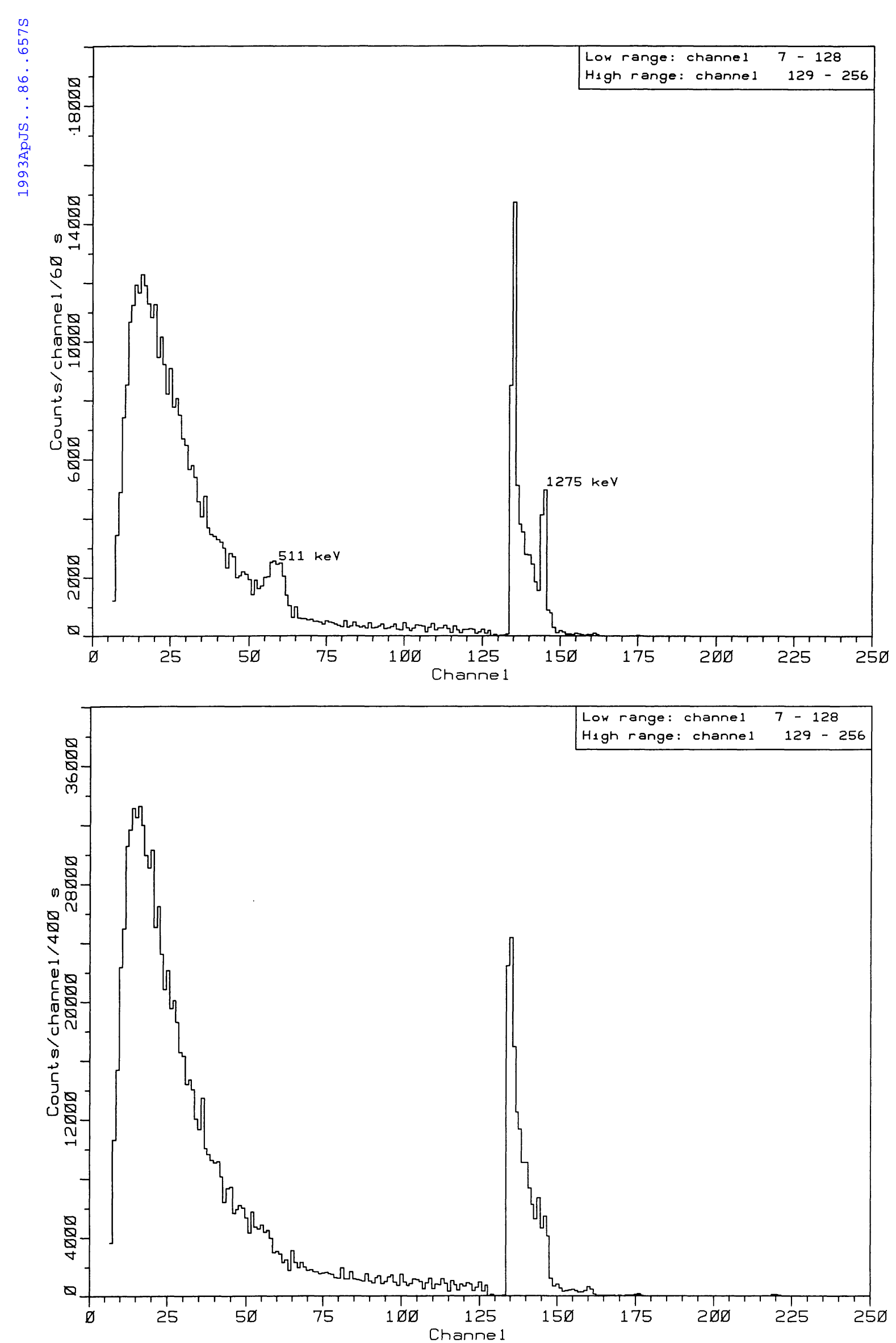

FIG. 35. $-{ }^{22} \mathrm{Na}$ measured at $\Theta=80^{\circ}, A=0^{\circ}$ at GSF (top panel, instrument level) and on $G R O$ level (bottom panel) 


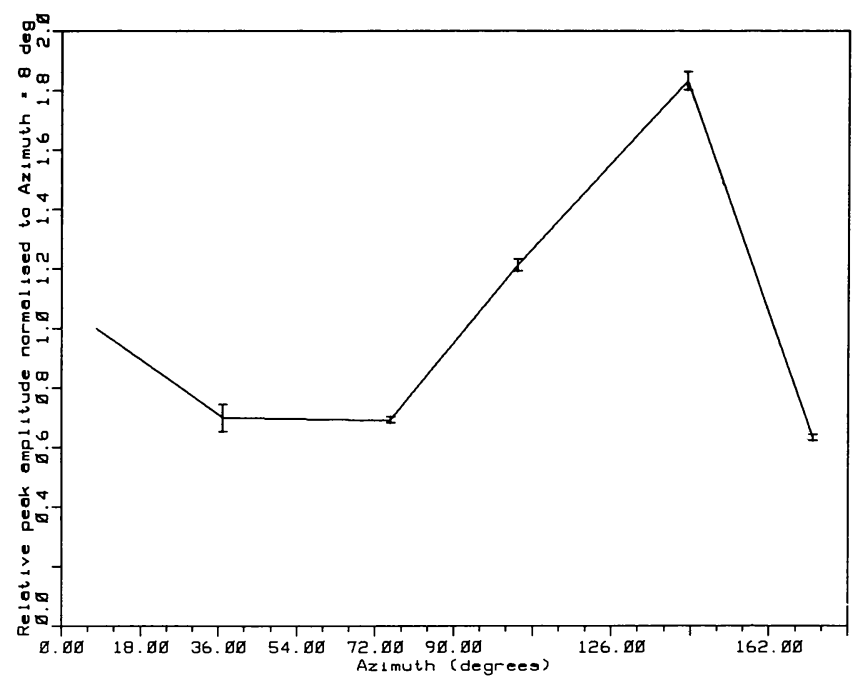

FIG. 36.- ${ }^{137} \mathrm{Cs}$ at $\Theta=45^{\circ}$, along azimuth

With COMPTEL in the solar-neutron mode, neutron interactions appear mostly in the gamma-2 channel, covering the TOF interval from about 8 to $40 \mathrm{~ns}$. The PSD and TOF criteria in this channel are such that solar neutrons incident on $D_{1}$ in the energy range from about 15 to $150 \mathrm{MeV}$ are recorded. In this energy interval COMPTEL can observe neutrons from about 14.5 to 55 minutes after release from the Sun. This corresponds to a minimum observed delay time of 6-47 minutes after the onset of the gamma-ray flash (assuming neutrons are not produced without accompanying gamma rays).

Neutron calibrations were performed on a COMPTEL prototype (Science Model III [SM III]) and on the full flight instrument. The COMPTEL prototype (SM III) consisted of two $D_{1}$ and three $D_{2}$ modules. This instrument was exposed at the Indiana University cyclotron facility to calibrated pulsed neutron beams from 20 to $200 \mathrm{MeV}$ incident at various angles with respect to the telescope axis. The neutrons were produced by the charge-exchange reaction ${ }^{7} \mathrm{Li}(p, n)^{7} \mathrm{Be}$, and each event

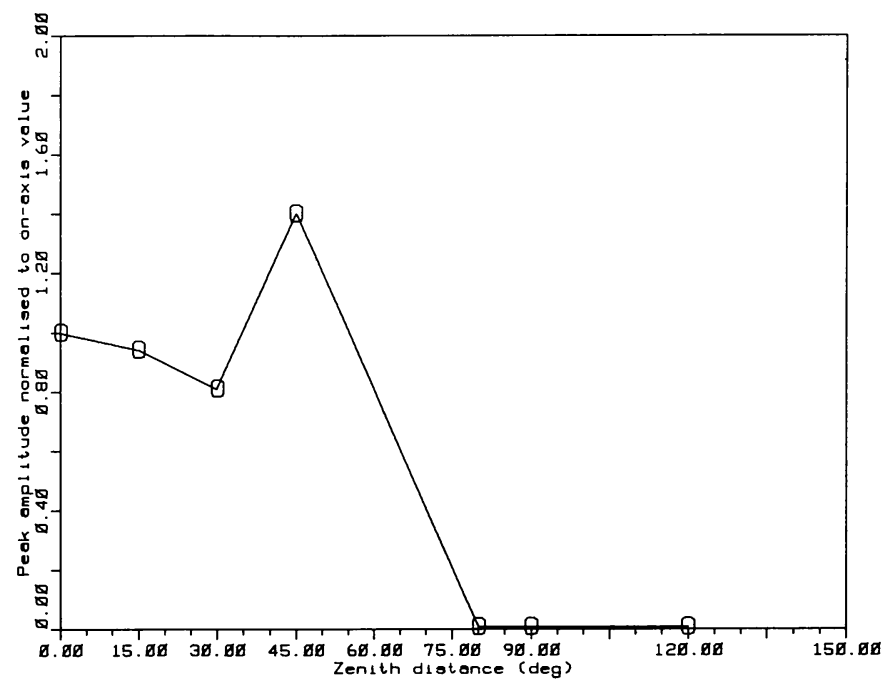

FIG. 37. ${ }^{133} \mathrm{Ba}$ at $A=0^{\circ}$, along zenith distance detected by the SM III was time-tagged to ensure selection of monoenergetic neutrons. The total fluence of neutrons was monitored independently. The resulting data were inspected to select events obeying the proper kinematic relationship for elastic scatters (eq. [2]). These events were counted and serve as the basis for estimating the telescope efficiency for detecting elastic scatters. The measured efficiencies of single $D_{1} / D_{2}$ module pairs (minitelescopes) are listed in Table 10 for some incident angles and energies. The resulting effective areas of COMPTEL for neutrons incident at $29^{\circ} .2$ are 12.7, 16.9, and $3.3 \mathrm{~cm}^{2}$ for energies of $18.5,35.7$, and $77.0 \mathrm{MeV}$, respectively, where the area has been scaled up to the full COMPTEL instrument from the single minitelescope efficiencies in Table 10. The efficiencies of Table 10 and the corresponding effective areas are crude values; they were derived merely from the count rate in the identifiable elastic scatter peak and do not rely upon the precise energy calibration or event location of individual events within each module.

Energy-resolution figures from a similarly crude analysis are available for the analyzed SM III data in order to illustrate the general features in the COMPTEL neutron response. In Figure 38 the measured energy spectra from monoenergetic neutron beams are indicated. Note the low-energy tail in the $77 \mathrm{MeV}$ data resulting from inelastic carbon reactions in $D_{1}$. The prototype SM III was not tuned for precise location of neutron interactions within the individual detectors, so the acceptance angle windows are unusually wide $\left( \pm 10^{\circ}\right)$. This wide acceptance window is determined here by the physical size of the detectors rather than the spatial resolution of the detectors. The energy resolution is largely determined by the TOF measurement between $D_{1}$ and $D_{2}$. The pulse height or energy resolution in the scintillation measurement in $D_{1}$ is negligible. There are three contributions to the energy resolution: the finite thickness and diameter of the two detectors creating an uncertainty in the path length over which the TOF is measured, the energy spread in the neutron beam due to the thickness of the target, and the electronic TOF resolution, which for COMPTEL is $1.5 \mathrm{~ns}$ (FWHM). For the minitelescope $\mathrm{D}_{1} / \mathrm{D}_{2}$ pair at $29^{\circ} 2$, for which no interaction location was performed, energy resolutions (FWHM) of $24 \%, 24 \%$, and $25 \%$ for 18.5 , 35.7 , and $77.0 \mathrm{MeV}$, respectively, were measured.

A more precise analysis of COMPTEL's response to neutrons was performed at 17.2 MeV. COMPTEL was exposed to

TABLE 10

ABSOLUTE MiNITELESCOPE EFFICIENCIES FOR NEUTRON DETECTION ${ }^{a}$

\begin{tabular}{rccc}
\hline \hline & \multicolumn{3}{c}{ ENERGY $(\mathrm{MeV})$} \\
\cline { 2 - 4 } ANGLE & 18.5 & 35.7 & 77.0 \\
\hline $5.5 \ldots \ldots$ & $\ldots$ & $\ldots$ & $6.7 \times 10^{-5}$ \\
$19.6 \ldots \ldots$ & $2.9 \times 10^{-4}$ & $4.4 \times 10^{-4}$ & $\ldots$ \\
$25.4 \ldots \ldots$ & $\ldots$ & $\ldots .0 \times 10^{-4}$ & $7.6 \times 10^{-5}$ \\
$29.0 \ldots \ldots$ & $3.1 \times 10^{-4}$ & $2.8 \times 10^{-4}$ & $5.4 \times 10^{-5}$ \\
$29.2 \ldots \ldots$ & $2.1 \times 10^{-4}$ & $1.2 \times 10^{-4}$ & $\cdots$ \\
$38.0 \ldots \ldots$ & $\ldots$ & $7.6 \times 10^{-5}$ & $\cdots$ \\
$44.0 \ldots \ldots$ & $\ldots$ & $\ldots$
\end{tabular}

${ }^{a}$ With the constraint that the inferred scatter angle $\theta_{n}$ be consistent $\left(\approx 10^{\circ}\right)$ with the neutron source direction. 

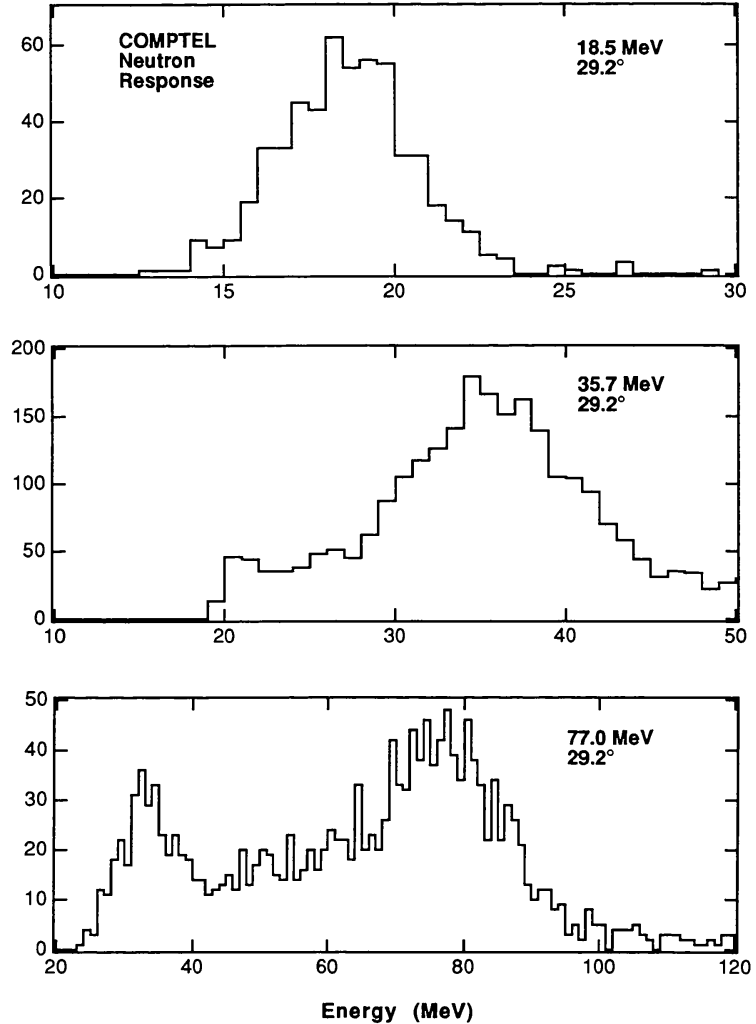

FIG. 38.-Measured energy spectra from monoenergetic neutrons at $18.5,35.7$, and $77.0 \mathrm{MeV}$.

a monoenergetic neutron source as part of the instrument calibration. At the GSF Van de Graaff accelerator the reaction $d(t, \alpha) n$ produced a range of neutron energies with a maximum of $17.2 \mathrm{MeV}$ in a steady state untagged beam. The resulting data can be compared with the SM III prototype data to verify the latter's accuracy. The neutron flux was monitored with a single-cell neutron detector, in this case the flight spare $\mathrm{D}_{1}$ module. The efficiency of the monitor was calculated by Monte Carlo techniques assuming a threshold of $3 \mathrm{MeV}$. The high threshold is necessary to exclude the counts due to the strong $2.223 \mathrm{MeV}$ from the thermalized neutron flux and to exclude the scattered neutron room background. The pulse heights in $D_{1}$, as part of the normal COMPTEL data processing, were converted to electron-equivalent energies. It was necessary to further translate these into proton-equivalent energies as described by Cecil, Anderson, \& Madey (1979) for the scintillator NE 213A. The energy of the scattered neutron was calculated from the time of flight. Nonrelativistic energies and scatter angles were calculated assuming that all reactions are simple elastic $(n, p)$ scatters. The location of the particle interactions within the detector modules were also calculated.

Figures 39 and 40 show (uncorrected for background) the measured energy and ARM distributions for a $17.2 \mathrm{MeV}$ neutron beam incident at $30^{\circ}$ from the telescope axis. Data were also taken at the same energy with the neutron beam parallel to the telescope axis. For the $30^{\circ}$ run the energy spectrum peaks at $17.1 \mathrm{MeV}$, close to the expected full energy peak at 17.18 $\mathrm{MeV}$. A low-energy tail exists and is most likely due to the fact that a degradation of neutron energies results from the ioniza-

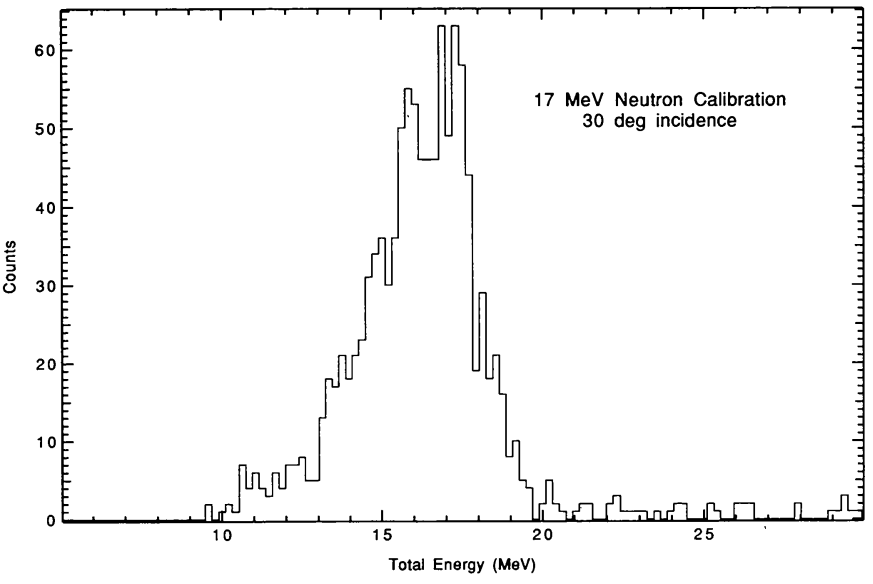

FIG. 39.-Measured energy spectrum of neutrons of $17 \mathrm{MeV}$

tion of the deuterons in the thick target. The FWHM of the peak is approximately $2.8 \mathrm{MeV}$, while the half-maximum point on the high side of the peak is only about $0.5 \mathrm{MeV}$ higher in energy than the peak position. The figure of $0.5 \mathrm{MeV}$ is a more appropriate measure of the instrument resolution than the FWHM due to the presence of the low-energy tail. The ARM distribution has a FWHM of approximately 4:9. The fact that the flight instrument $17 \mathrm{MeV}$ ARM distribution is narrow confirms that the energies are being calculated correctly. Incorrect energy assignments result in incorrect ARM values: that is, the geometrical and kinematic constraints can only be satisfied if the energy assignments are correct. The width of the ARM distribution, the HWHM (on the high side) of the energy peak, and the predicted energy uncertainty due to the path-length variation between $D_{1}$ and $\mathrm{D}_{2}$ are self-consistent, assuming that the spatial resolution of neutron-event positions within the modules is similar to that of gamma rays. In other words, the TOF error resulting from the finite thickness of the $D_{1}$ and $D_{2}$ modules produces an error in the measured scattered neutron energy, which in turn produces an error in the calculated scatter angle.

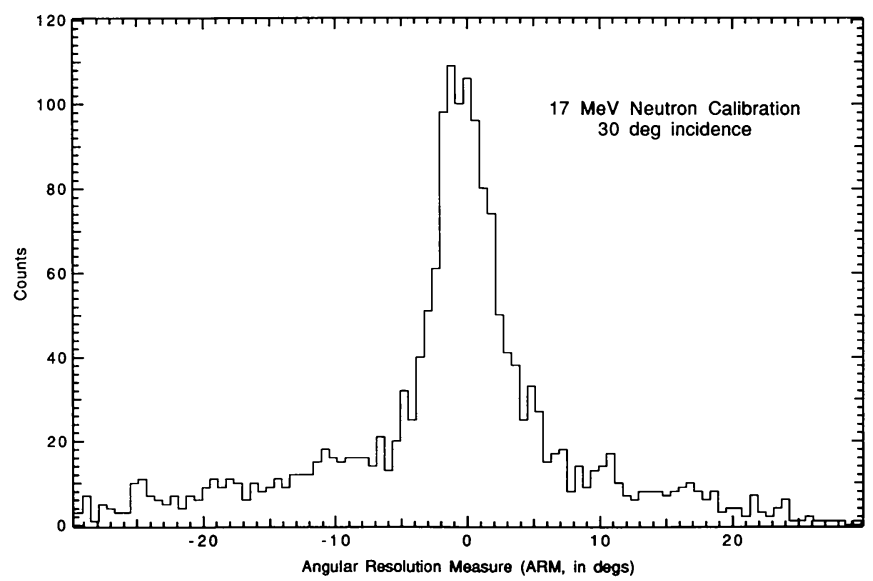

FIG. 40.-Angular resolution measure (ARM) distribution of $17 \mathrm{MeV}$ neutrons. 


\section{DEMONSTRATION OF COMPTEL IMAGING CAPABILITIES}

\subsection{Maximum-Entropy Imaging of COMPTEL Data}

Studies of the maximum-entropy method (MEM) applied to spark-chamber and Compton telescopes have been described previously by Strong \& Diehl (1988), and an update for COMPTEL was given by Strong et al. (1989). The Compton telescope has always been an excellent candidate for MEM, not only because no other method has proved able to provide sky maps in the strict sense of intensity distributions consistent with the data (other methods give various types of probability distributions which cannot be interpreted as intensities) but also because of the nature of the instrumental data. The data are characterized by the three-dimensional point-spread function. The "data space" is similarly three-dimensional ( or more if energy resolution is included). Since we want to generate two-dimensional sky maps, it is clear that our "data space" is very different from our "image space." This is just the kind of problem where MEM is effective; in fact, the more different the data and image spaces are, the more useful are the so-called indirect imaging methods. One interesting aspect of Compton telescope data is that the Compton scatter "dimension" represents an oversampling of the image; it is as if we observed the sky with many separate two-dimensional PSFs and attempted to use them all simultaneously for the reconstruction. One advantage of having very different image and data spaces and of oversampling is that unwanted correlations between image and data (e.g., the image following point-to-point statistical variations in the data) are practically eliminated.

The software package used is MEMSYS2 from Maximum Entropy Data Consultants, Ltd. The user provides a function which computes the response to any given input image; the package then allows an iterative solution for the maximum-entropy image which is consistent with the data provided. The response representation given by equation (4) in $\S 6.1$ enables a fast computation of the response to any given input image, provided that the convolution with the PSF can be computed quickly; in practice we have used a fast-Fourier transform (FFT) method (in particular, Winograd FFT, which is not restricted to powers of 2 ) which treats the spherical coordinate system as a locally flat Cartesian system. Unfortunately, this approximation is increasingly poor as we go to larger scattered angles (characteristic of lower photon energies); but explicit "brute force" computation of the convolution is at present ruled out, since it involves very large computer resources.

For clarity we have omitted the energy dependence of terms in equation (4); however, it should be clear that in a real application an additional convolution over the input intensity spectrum is required and that the PSF is an energy-dependent function.

Results using calibration sources.-The calibration of COMPTEL included sources with energies from 0.66 to 20 $\mathrm{MeV}$ (see Table 5). These data can be used as a test of the MEM deconvolution, although the finite distance of the source (about $8 \mathrm{~m}$ ) means some spreading of the image. This can be minimized by using data from only one $\mathrm{D}_{1}$ detector; the spread is then about $2^{\circ}$. This problem does not, of course, arise in flight. Since the sources used were quite strong, the signalto-background ratio is not typical of flight situations, so it is necessary to add in data from background runs to obtain more realistic tests. Data for different source positions can be combined to simulate more than one source in the field of view and test the spatial resolution for source separation as a function of signal-to-background ratio.

We concentrate on one source energy, $6.1 \mathrm{MeV}$, and a configuration of three sources separated by $10^{\circ}$ and two sources separated by $5^{\circ}$. The source counts are typical for a Crab-like source and a COMPTEL observation of 2 weeks. Table 11 summarizes the cases presented here.

For the ideal case of no added background (Fig. 41) the sources are completely resolved and in the correct positions, with no artifacts.

If the background is increased to 5 times the counts for one source (Fig. 42), the sources are still well imaged but the contrast is smaller. Edge effects become noticeable: some parts of the image near the edge of the field are not constrained by the data and are assigned the "default" value defined by the user.

A test of the angular resolution is shown in Figure 43, where two sources separated by $5^{\circ}$ are used. The sources are clearly resolved, but there is significant fill-in between the sources; the intrinsic angular width $\left(2^{\circ}\right)$ due to the nonparallel source beam ( see above) contributes to this, as does the bin size used, $1^{\circ}$ in $(\chi, \psi)$ and $2^{\circ}$ in $\bar{\varphi}$, which is really too large to exploit the PSF fully. Considering both these effects, the source resolution for strong sources is probably better than $5^{\circ}$.

All of these tests were made with just one $D_{1}$ module to avoid source spreading; this also has the effect of reducing the coverage of the geometrical response $(g)$ in equation (4). In flight all $\mathrm{D}_{1}$ modules are used (sources at infinity), and the geometrical response is in this case more uniform.

As a conclusion to this section, we give an example using actual COMPTEL in-flight data, showing the real performance for the signal-to-background ratio, which holds for a strong source (Fig. 44). The data consist of 10 days from the "validation" period at the beginning of the mission, during which the scientific performance of the instrument was checked. The $\mathrm{Crab}$ (nebula and pulsar) dominates this field. The energy range used is $1-30 \mathrm{MeV}$, the bin size is $1^{\circ}$ in $\chi$ and $\psi$ and $2^{\circ}$ in $\bar{\varphi}$. The image contains $2.7 \times 10^{5}$ counts, of which about $10^{4}$ come from the Crab. The image shown incorporates an improvement in the application of the maximum-entropy method, which allows an estimate of the background to be included. In this case the background was derived by averaging the data so that the $\bar{\varphi}$-distribution is independent of $\chi$ and $\psi$, thus removing any source signature. The PSF used is that appropriate to a source with an $E^{-2}$ spectrum. The good performance of the method in flight is clear from the very strong peak at the Crab position, with the surrounding field being almost flat.

TABLE 11

DATA USED For MAXIMUM-ENTROPY IMAGES OF CALIBRATION SOURCES

\begin{tabular}{lcccr}
\hline \hline & $\begin{array}{c}\text { Source } \\
\text { Energy } \\
(\mathrm{MeV})\end{array}$ & $\begin{array}{c}\text { Source } \\
\text { Configuration } \\
\text { Angles from } \\
\text { Axis }\end{array}$ & $\begin{array}{c}\text { Counts } \\
\text { per } \\
\text { Source }\end{array}$ & $\begin{array}{c}\text { Total } \\
\text { Counts }\end{array}$ \\
\hline $41 \ldots$ & 6.1 & $0^{\circ}, 10^{\circ}, 20^{\circ}$ & 1470 & 4424 \\
$42 \ldots$ & 6.1 & $0^{\circ}, 10^{\circ}, 20^{\circ}$ & 1470 & 12408 \\
$43 \ldots$ & 6.1 & $0^{\circ}, 5^{\circ}$ & 1470 & 3480 \\
\hline
\end{tabular}




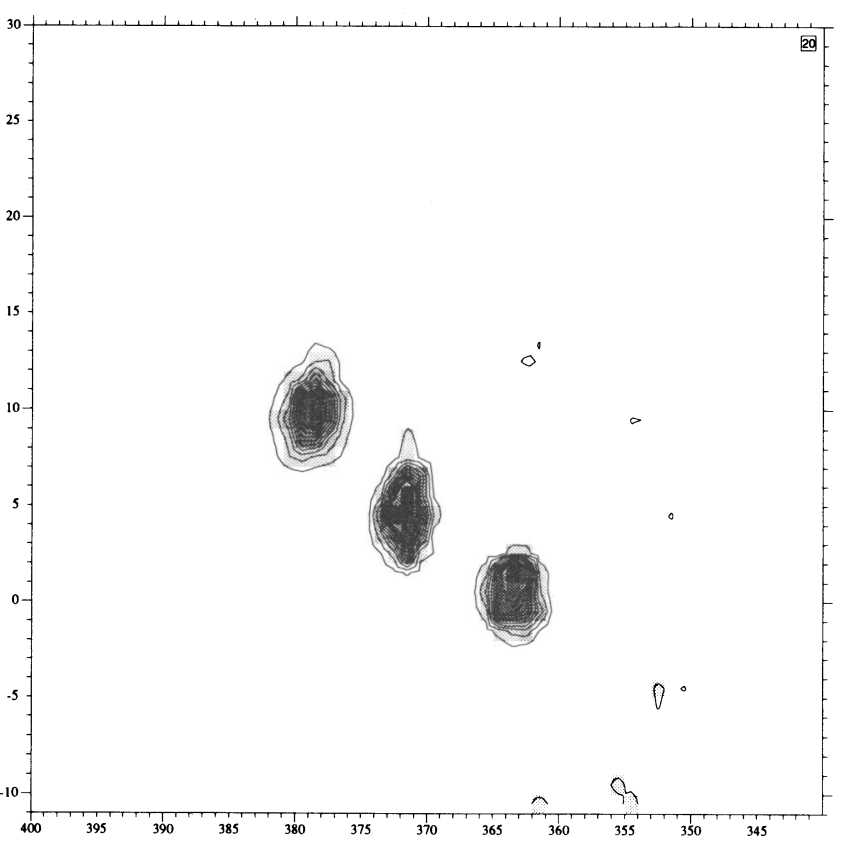

FIG. 41.-MEM images of three $6.1 \mathrm{MeV}$ calibration sources separated by $10^{\circ}$, without added background. Details are given in Table 11 . Coordinates are in degrees.

\subsection{Maximum-Likelihood Method for COMPTEL Source Recognition and Parameter Estimation}

\subsubsection{Description of the Method}

The maximum-likelihood method was introduced for data analysis in gamma-ray astronomy by Pollock et al. (1981), who searched the $C O S B$ data (energy range $50 \mathrm{MeV}-5 \mathrm{GeV}$ )

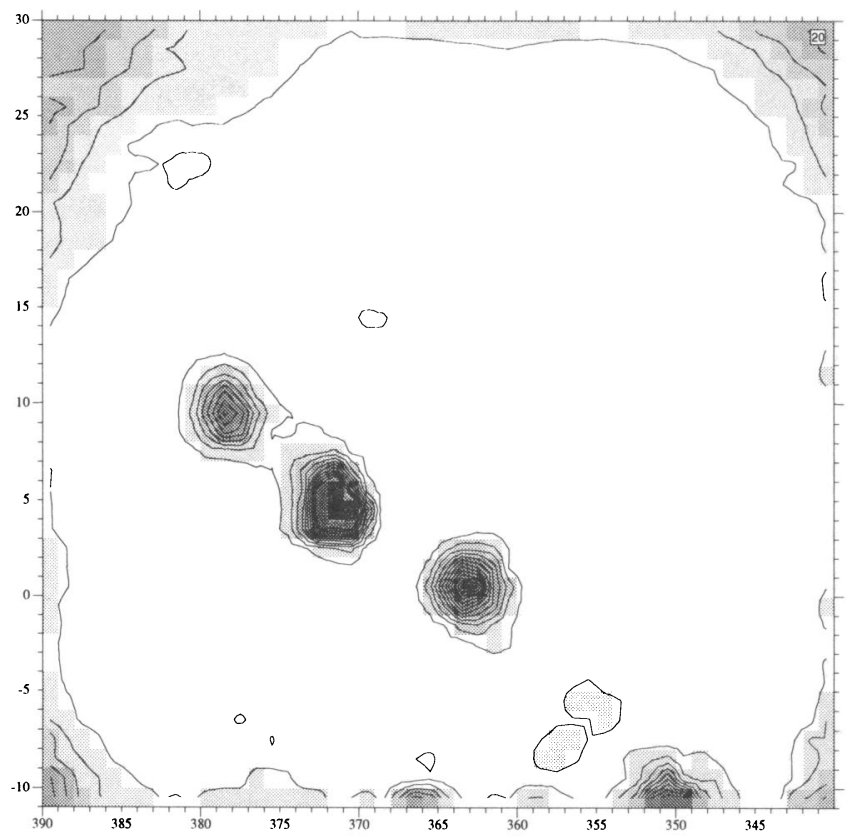

Fig. 42.-Adding a significant amount of background reduces the quality of the image, but the sources are clearly visible.

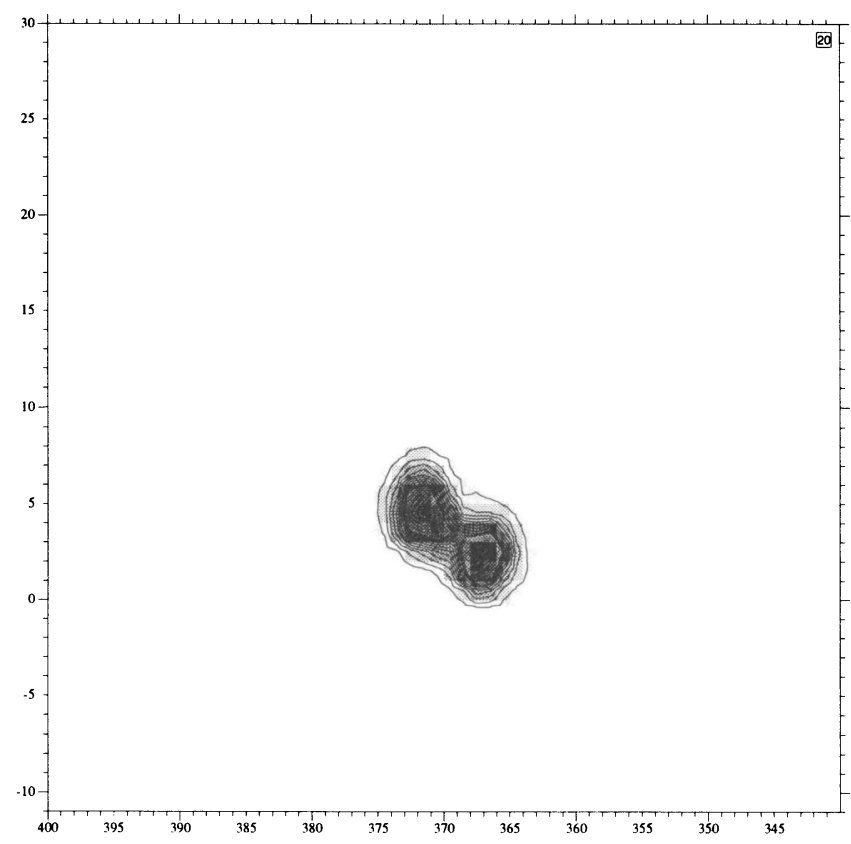

FIG. 43.-Two sources separated by $5^{\circ}$ are resolved; the intrinsic source diameter of $2^{\circ}$ contributes to the overlap, so the true resolution is better than is apparent here.

for extragalactic gamma-ray sources. Pollock et al. (1985) applied maximum likelihood to the search for point sources in excess to a model for the diffuse gamma-ray background. They compared the likelihood that the data resulted from the diffuse model alone to the likelihood that the data resulted from the diffuse model plus point sources. These applications to $\operatorname{COS} B$ data at higher gamma-ray energies could be performed in the two-dimensional $\operatorname{COS} B$ data space. In the COMPTEL case, the maximum-likelihood method requires a three-dimensional data space and a three-dimensional response function as does the maximum-entropy method as described above (§6.1.).

The maximum-likelihood method can be summarized as follows: assuming an a priori knowledge of a model of the

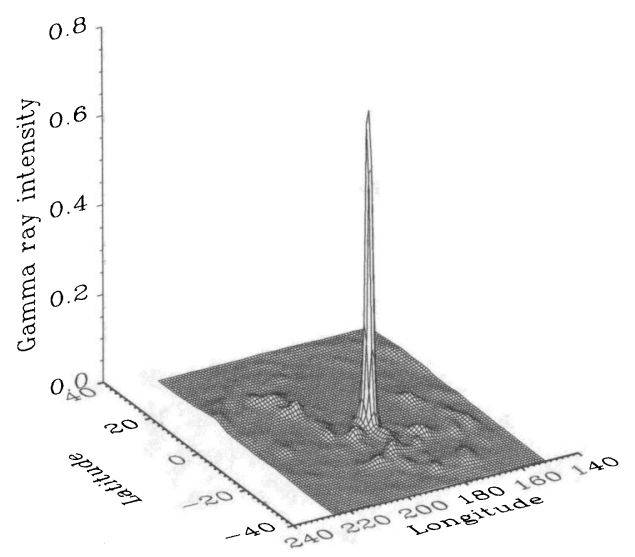

FIG. 44.-Maximum-entropy image of 10 days of flight data from the anticenter region of the Milky Way. The Crab stands out clearly. 
measured quantities, hypothesis $H_{0}$ containing $p$ free parameters, the maximum-likelihood analysis determines the parameter values which give the most likely representation of the measured quantities, maximizing the likelihood over all bins in data space, $L\left(H_{0}\right)$. If one wishes to verify whether the data support the presence of additional model components, then one can enhance the model with these component (s), hypothesis $H_{1}$ with $p+q$ parameters, and again determine the maximum likelihood $L\left(H_{1}\right)$. Then the likelihood ratio $L\left(H_{0}\right) /$ $L\left(H_{1}\right)$ can be interpreted as the degree to which the data support hypothesis $H_{0}$ against $H_{1}$ (Edwards 1972). In fact, in a classical interpretation $\lambda=2 \log \left[L\left(H_{1}\right) / L\left(H_{0}\right)\right]$ is distributed as $\chi_{q}^{2}$ under the null hypothesis, with $q$ the number of free parameters that are added to the null hypothesis.

A practical example for the COMPTEL analysis is the search for sources on top of an assumed background structure, e.g., due to Galactic diffuse emission and the instrumental background. The latter distributions are convolved into the threedimensional data space using the COMPTEL response functions, rendering hypothesis $H_{0}$ with free parameters for the intensities of its components. Furthermore, for a trial source position $\left(\chi_{0}, \psi_{0}\right)$ the known response for a point source can be added to the model, giving hypothesis $H_{1}$ by adding three additional parameters $\left(\chi_{0}, \psi_{0}\right.$, and flux $)$. The log-likelihood ratio $\lambda$ behaves as $\chi_{3}^{2}$ under the null hypothesis. Therefore, one obtains a 99\% confidence detection if $\lambda>11.3$. If one accepts $H_{1}$, confidence levels can be generated on both the source position and the source flux. A source which is significant "beyond reasonable doubt" can be added to the background model at its most likely position (for more details see de Boer et al. 1991 ). It should be noted, however, that if one searches a large sky area, one should take into account the effective number of trials made. This can be estimated by dividing the sky area by the effective area of the response function. A verification can be obtained by analyzing Monte Carlo simulations of the COMPTEL measurements (see, e.g., Mattox 1990 for the case of spark-chamber gamma-ray telescopes).

Once a source detection has been accepted, its gamma-ray energy spectrum can be determined by applying the maximum-likelihood analysis in differential energy ranges. For each energy interval an accurate three-dimensional response function is required which is a function of the source spectral shape. If the statistics allow the choice of sufficiently narrow energy intervals, the required three-dimensional response functions become rather insensitive to the assumed input spectra. In the case of smooth spectra, an accurate deconvolved source spectrum can be derived in a few iterations. If spectral line features are to be studied, then the full details of the response function and model spectra have to be considered.

\subsubsection{Results from Simulations}

Instead of using calibration data, as was done for the verification of the maximum-entropy method, we simulated data spaces for arbitrary model intensities. Specifically, the calibration sources do not appear as ideal point sources, owing to their finite distances. Furthermore, we wished to control our input image completely, in order to be able to verify the accuracy of the parameter estimation (e.g., significance of detection, source positions, fluxes). Note the distinction between the maximum-likelihood method and the maximum-entropy method. Whereas the aim of the maximum-likelihood method is to determine source parameters, the aim of the maximumentropy method is primarily the production of images.

In this section we will present the results of some simulations performed with the empirically determined PSF at $6.13 \mathrm{MeV}$. First, we show a verification of the calculation of the detection significance, namely, that the likelihood ratio $\lambda$ behaves as $\chi_{3}^{2}$. Figure 45 confirms this behavior for a search for sources over a sky area of $6000 \mathrm{deg}^{2}$ in a data space in which only background distributions have been simulated. The integral distribution of random "sources" (local excesses in the $\lambda$-map on top of a modeled background distribution) behaves indeed as $\chi_{3}^{2}$. Therefore, if we know a priori the position of an object, then $\lambda>11.3$ at that position renders a source detection at a $99 \%$ confidence level. Furthermore, the measured and predicted integral distributions in Figure 45 can be normalized for an effective number of trials between 127 and 153. This means that a single trial corresponds for this energy to a sky area with radius between 3.5 and 4.0 , roughly consistent with the resolution of the instrument. Consequently, in a search for unknown sources over an area of sky, we have to take this into account.

Second, we present results which indicate the accuracy of the parameter estimation (positions and fluxes). Source simulations have been performed in which the number of counts is typical for the integrated energy range of 3-12 MeV in the flight situation. The background was assumed to comprise about $10^{5}$ photons for the quoted energy range and a full observation period of $\approx 4 \times 10^{5} \mathrm{~s}$ effective integration time. (The actual measured COMPTEL background in orbit is about 6 times smaller; see Table 12.) The data spaces used for the likelihood results are $79^{\circ} \times 79^{\circ} \times 40^{\circ}$ in $(\chi, \psi, \bar{\varphi})$ with $1^{\circ}$ bins along each dimension. The likelihood map displayed in Figure 46

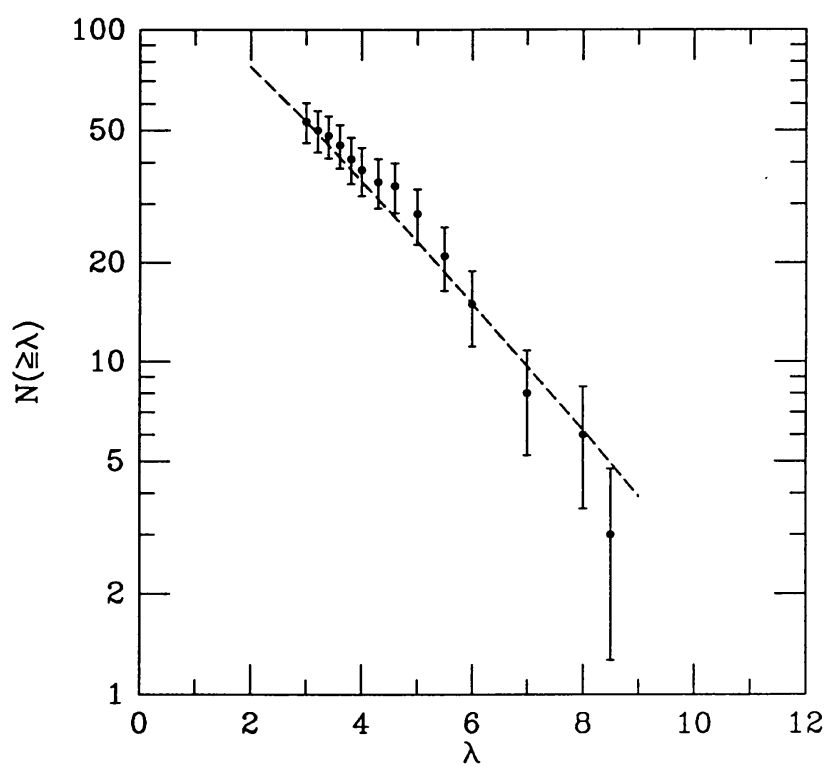

FIG. 45.-- Integral distribution of the number of random "sources" detected in a search for sources in a data space containing only simulated background distributions. The searched sky area is $6000 \mathrm{deg}^{2}$. The broken line indicates the expected distribution if $\lambda$ behaves as under the null hypothesis, and for 135 trials. 


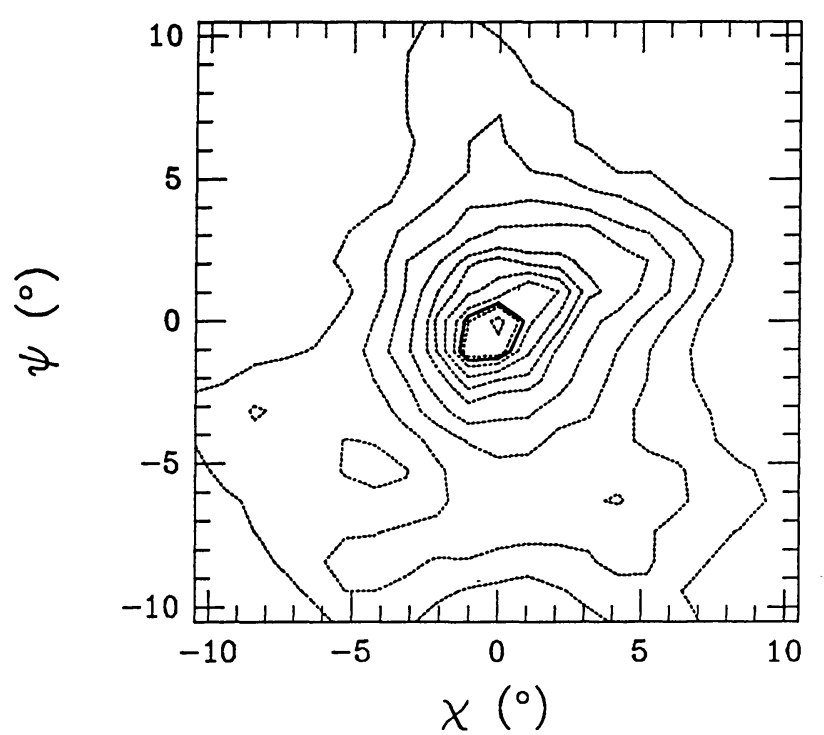

FIG. 46.-Sample likelihood ratio map $(\lambda)$ for a simulated source observation (for details see text); contour levels: $20,30,40,50,60,65,70,75$, 80 , and 85 . The $99 \%$ confidence level on the measured source position is indicated by the solid contour.

shows the case of a simulated on-axis source [at $(\chi, \psi)=(0$, 0 )], with about 1100 source counts, whereas the $\approx 10^{5}$ background counts are distributed as they would be for an isotropic sky. For comparison, about 6000 source counts are expected from the Crab total emission in the energy range 3-12 MeV in a single observation period. Figure 46 undoubtedly proves with $\lambda_{\max }=87$ the detection of a point source. Furthermore, for this example of $1 \%$ source counts on about $10^{5}$ background counts, the formal positional accuracy is about $2^{\circ}$ for a $99 \%$ confidence level (indicated in the figure). This resolution quickly improves with the number of counts, e.g., for $6 \%$ source counts it is about $0: 3$. For such a well-resolved source the relative error in flux is typically less than $5 \%$.

\section{COMPTEL SENSITIVITY}

\subsection{In-Orbit Background}

Since a Compton telescope was never flown on a satellite before the launch of $G R O$, the prelaunch estimate of the in-orbit background rate (based on data from balloon flights) contained many uncertainties. The estimate of the COMPTEL background rate had been made from the event rate measured with the MPE Compton telescope (Schönfelder et al. 1982) during a balloon flight in 1982 from Uberaba, Brazil (von Ballmoos, Diehl, \& Schönfelder 1987). The Uberaba flight was chosen, because its geographical latitude of $20^{\circ}$ south provides a better approximation to the $28^{\circ}$ inclination $G R O$ orbit than Palestine, Texas, with its latitude of $32^{\circ}$ north.

The predicted rates can now be compared with the directly measured in-flight background rates. The background rate can be influenced in a very drastic way by event-selection criteria. The most critical selection parameters are the energy thresholds in $D_{1}$ and $D_{2}$, the time-of-flight window of accepted events, and the rejection criteria for Earth albedo events. At the time of writing this paper ( 1 year after the launch of $G R O$ ), a certain set of selection criteria had been defined to optimize the performance of COMPTEL. It may be further modified in the future.

The selections made were the following:

1. $E_{1}>70 \mathrm{keV}$.

2. $E_{2}>650 \mathrm{keV}$.

3. $\varphi<50^{\circ}$.

4. TOF window: channels 115-130.

5. PSD window: channels 0-110.

6. Minimal angular distance between Earth horizon and event circle: $10^{\circ}$.

If only those events are accepted whose event circles passed within the $3 \sigma$-wide acceptance interval around the source direction of a celestial on-axis source (see Fig. 28), then the background sums listed in Table 12 are obtained in a 2 week observation period. For Table 12 the $G R O$ viewing period $I$ (anticenter of the galaxy) was chosen. Other event selections are those listed above. The event rates of other viewing periods are similar, if interpreted over the entire 2 week period (deviations less than $30 \%$ ), although the orbit variations due to the changing geomagnetic cutoff conditions are as high as a factor of 2 .

These rates are about 4 times higher than originally estimated from the balloon flight data (after scaling to the COMPTEL size and after correcting for the missing atmospheric background). A detailed study of this discrepancy and an analysis of the various background components which might contribute is in progress. Part of the discrepancy may be due to neutron-induced events ( see Morris et al. 1992).

A typical background spectrum integrated over a 2 week observation period is plotted in Figure 47. The spectrum shows two instrumental background lines, the $1.46 \mathrm{MeV}$ line from ${ }^{40} \mathrm{~K}$ and the $2.23 \mathrm{MeV}$ line from neutron capture in $\mathrm{D}_{1}$.

\subsection{Point-Source Sensitivity}

The $3 \sigma$ point-source sensitivity for an on-axis source is in good approximation given by

$$
F_{3 \sigma}=\frac{3\left(N_{\mathrm{BGD}}+N_{\text {source }}\right)^{1 / 2}}{A_{\mathrm{eff}} T_{\mathrm{eff}}},
$$

where $N_{\text {BGD }}$ and $N_{\text {source }}$ are the numbers of background and source events within the source acceptance element (note that $\left.N_{\text {source }} \ll N_{\text {BGD }}\right), A_{\text {eff }}$ is the effective detection area, and $T_{\text {eff }}$ is

TABLE 12

ACTUAL IN-FLIGHT COMPTEL BACKGROUND RATE

\begin{tabular}{cc}
\hline \hline $\begin{array}{c}E_{\gamma} \\
(\mathrm{MeV})\end{array}$ & Number of Events \\
\hline $0.7-1 \ldots \ldots$ & 9600 \\
$1-3 \ldots \ldots \ldots$ & 51000 \\
$3-10 \ldots \ldots$ & 16900 \\
$10-30 \ldots \ldots$ & 1150 \\
\hline
\end{tabular}

${ }^{a}$ Within 2 weeks of observation for fully operational telescope $\left(\bar{\varphi}<50^{\circ}\right)$. 


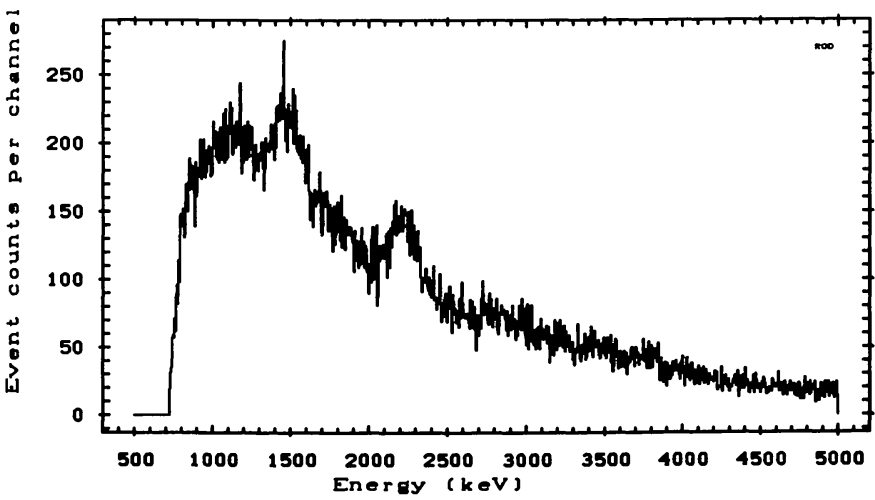

FIG. 47.-COMPTEL background spectrum for a 2 week observation period. ( $G R O$ viewing period 4 [high Galactic latitude around NGC 4151] was chosen). Events within a $6^{\circ}$ wide acceptance interval around the onaxis direction are accepted. Other event selections were those defined in $\S 8.1$. The channel width on the ordinate is $10 \mathrm{keV}$.

the mean effective observation time. (In an imaging telescope like COMPTEL the background can be deduced from several "off bins"; therefore, eq. [7] is in good approximation valid; the expression with exponent $\frac{1}{2}$ is not $2 N_{\mathrm{BGD}}+N_{\text {source }}$.) $T_{\text {eff }}$ is mainly determined by the Earth occultation of the source; it especially depends on the elevation angle of the source from the orbital plane and from the constraint on the minimal angular distance between the Earth horizon and each measured event circle. The impact of the latter constraint is an energy dependence of $T_{\text {eff }}$, since the mean scatter angle $\varphi$ decreases with increasing energy. On average, $T_{\text {eff }}$ is about $33 \%$ of 14 days $=4 \times 10^{5} \mathrm{~s}$ for the rejection criterion of Earth horizon events as defined above $(\S 8.1)$.

In the final COMPTEL data analysis, the energy-dependent exposure of COMPTEL (product of detection area and observation time) to a celestial point source is calculated as a function of time. To estimate the mean COMPTEL sensitivity limit, the following approach was taken.

The observation of the Crab by COMPTEL was considered to provide a typical effective exposure value. The Crab exposure was derived from the measured Crab counts, assuming a total Crab emission of $3 \times 10^{-3} E_{\mathrm{MeV}}^{-2.3}$ photons $\mathrm{cm}^{-2} \mathrm{~s}^{-1} \mathrm{MeV}^{-1}$ (Graser \& Schönfelder 1982). Using this typical effective exposure, the $3 \sigma$ sensitivity limit was derived from equation (7), with $N_{\text {BGD }}$ from Table 12 .

Table 13 summarizes the mean effective exposure factors and the derived sensitivity limits of the fully operational

TABLE 13

$3 \sigma$ Point-Source ContinuUm Sensitivity WITHIN 2 WEEKS

\begin{tabular}{ccc}
\hline \hline $\begin{array}{c}E_{\gamma} \\
(\mathrm{MeV})\end{array}$ & $\begin{array}{c}\text { Effective } \\
\text { Exposure } \\
\left(\mathrm{cm}^{2} \mathrm{~s}\right)\end{array}$ & $\begin{array}{c}F_{3 \sigma} \\
\left(\mathrm{cm}^{-2} \mathrm{~s}^{-1}\right)\end{array}$ \\
\hline $0.7-1 \ldots \ldots$. & $2.3 \times 10^{6}$ & $1.2 \times 10^{-4}$ \\
$1-3 \ldots \ldots \ldots$ & $3.9 \times 10^{6}$ & $1.7 \times 10^{-4}$ \\
$3-10 \ldots \ldots$. & $7.1 \times 10^{6}$ & $5.5 \times 10^{-5}$ \\
$10-30 \ldots \ldots$ & $10.8 \times 10^{6}$ & $9.4 \times 10^{-6}$ \\
$1-30 \ldots \ldots$ & $4.8 \times 10^{6}$ & $1.6 \times 10^{-4}$ \\
\hline
\end{tabular}

COMPTEL for a 2 week observation period. These limits are consistent with the fluxes derived for the weak quasars 3C 273 and 3C 279 (Hermsen et al. 1993), using the maximum-likelihood method of $\S 7.2$.

In Figure 48 the presently achievable point-source sensitivities are compared with spectra from a few known active galactic nuclei.

In the same manner, the $3 \sigma$ gamma-ray line sensitivity of an on-axis point source was calculated (see Fig. 49). The line widths were taken from Figure 30. The acceptance circle for events around the source position had a radius of $3^{\circ}$. For a 2 week observation period the sensitivity curve of Figure 49 was derived from Figure 47.

\subsection{Sensitivity to Burst Detection}

COMPTEL measures cosmic gamma-ray burst events primarily via the "single-detector mode" ( see $\S 6.2$ ). In addition, if the burst occurs within the imaging field of view, then COMPTEL can also measure the burst using data from its "telescope mode"; this provides an image of the burst and its subsequent source location. Since both modes are scientifically useful, we discuss the sensitivity of each mode below.

\subsubsection{Double-Scatter Mode}

The angular resolution of a point source in this mode is determined by the spatial and energy resolution of the detectors. Let $S(>E)$ be the burst fluence $\left(\mathrm{ergs} \mathrm{cm}^{-2}\right)$; then the lower detection threshold is given by

$$
S(>1 \mathrm{MeV})_{\text {low }}=\frac{E N(>1 \mathrm{MeV})}{A_{\text {eff }}},
$$

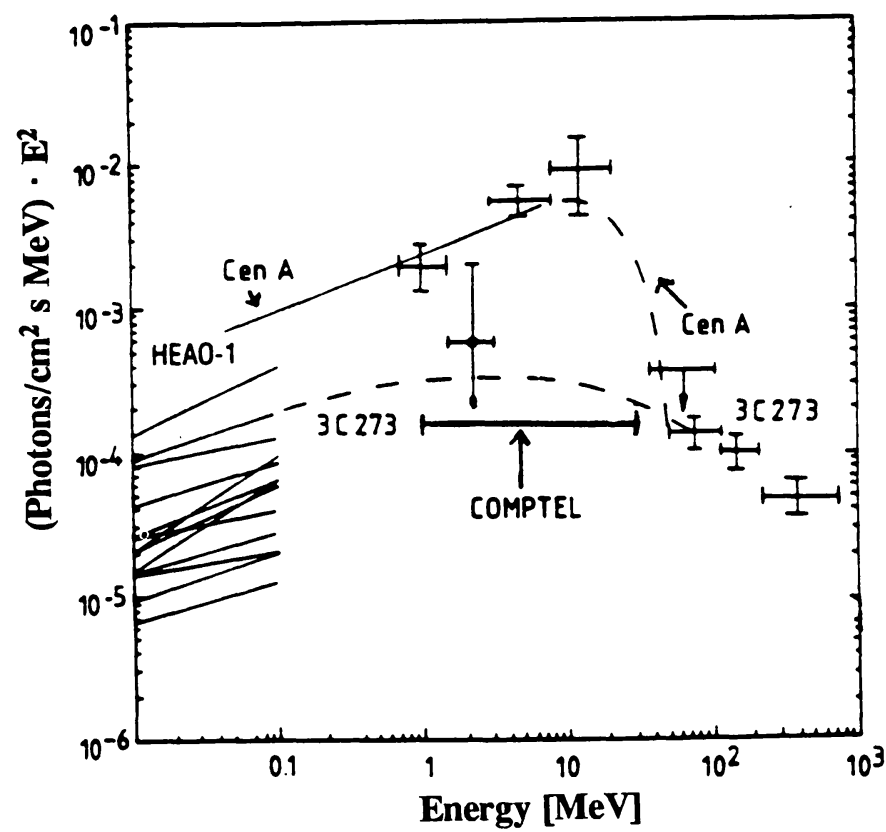

FIG. 48.-The $3 \sigma$ continuum point-source sensitivity of COMPTEL within 2 weeks of observation. A few spectra of active galactic nuclei are shown for comparison (Rothschild et al. 1983; von Ballmoos et al. 1987; Hermsen et al. 1981; Bezler et al. 1984). 


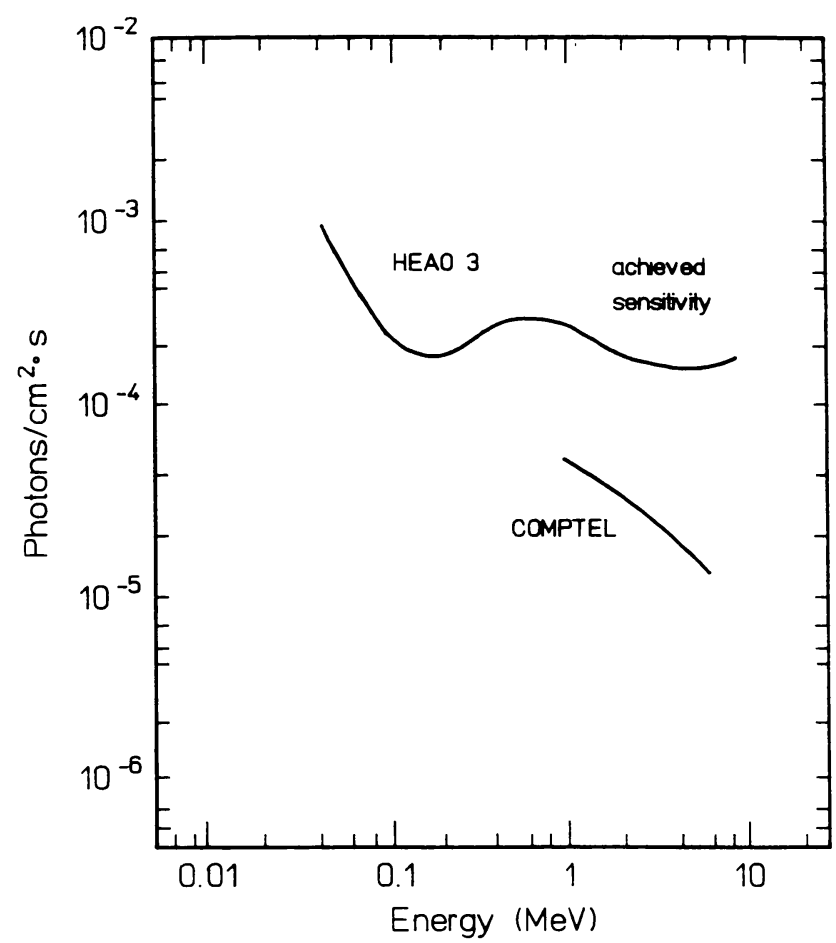

FIG. 49.-The $3 \sigma$ gamma-ray line point-source sensitivity of COMPTEL (on-axis, 2 weeks of observation of fully operational COMPTEL). The achieved $H E A O 3$ sensitivity is shown for comparison (Mahoney et al. 1980).

where $E$ is the mean photon energy ( $5 \mathrm{MeV}$ ) over the energy range $1-30 \mathrm{MeV}$ (assuming a power-law spectrum) and $A_{\text {eff }}$ is the effective detection area for totally absorbed on-axis events. If we require $N(>1 \mathrm{MeV})=30$ events using $A_{\text {eff }}=30 \mathrm{~cm}^{2}$, we obtain $S_{\text {low }}(>1 \mathrm{MeV})=8 \times 10^{-6} \mathrm{ergs} \mathrm{cm}^{-2}$. The number of background events within a $4^{\circ} \times 4^{\circ}$ resolution element is about 0.2 counts $\mathrm{s}^{-1}$ (derived from Table 12). Therefore, the detection of bursts is photon-limited, not background-limited. An upper limit to the burst threshold in this mode is defined by the rate of chance-coincidence events between $D_{1}$ and $D_{2}$. Using GRB 840805 (Share et al. 1986) with $S(>20 \mathrm{keV})=3 \times$ $10^{-3} \mathrm{ergs} \mathrm{cm}^{-2}$, this rate can be estimated to 2000 counts s $\mathrm{s}^{-1}$. Therefore, bursts of similar duration ( $45 \mathrm{~s})$ and spectral shape can only be studied if $S \leq 10^{-4}$ ergs $\mathrm{cm}^{-2}$.

$S M M$ observations show that emission above $1 \mathrm{MeV}$ is a common feature of gamma-ray bursts (e.g., Matz et al. 1985). Assuming an isotropic burst-event distribution, the rate of high-energy events detected by SMM, the COMPTEL burst threshold (see above), and the distribution of events in the log $N / \log S$ curve (e.g., Liang \& Petrosian 1986, Fig. 1.15), we estimate that COMPTEL should be able to detect $\approx 10-20$ bursts $\mathrm{yr}^{-1}$ within its field of view of $1 \mathrm{sr}$. This estimate is consistent with the observed rate of bursts within the COMPTEL field of view within the first year of the mission. Depending on the fluence, burst locations are accurate to about $1^{\circ}$ at 1-10 MeV gamma-ray energies.

\subsubsection{Single-Detector Mode}

Continuum sensitivities. - Using the background spectrum estimate for BATSE (Fishman et al. 1982), $5 \sigma$ continuum sensitivities of burst fluence $S$ ( $\mathrm{ergs} \mathrm{cm}^{-2}$ ) for four energy ranges and four typical burst durations were calculated. Mean photon energies per energy range were derived using two typical continuum shapes for burst events: a thermal bremsstrahlung model $(k T=250 \mathrm{keV})$ for energies below $300 \mathrm{keV}$ and a power-law model $\left(E^{-2}\right)$ for energies above $300 \mathrm{keV}$. The numbers of burst photons were calculated according to Li \& Ma (1983) from

$$
\Sigma=\frac{N_{\text {on }}-\alpha N_{\text {off }}}{\left[\alpha\left(N_{\text {on }}+N_{\text {off }}\right)\right]^{1 / 2}},
$$

where significance $\Sigma=5 \sigma$; the number of burst photons is $N_{s}=N_{\text {on }}-\alpha N_{\text {off }} ; N_{\text {on }}=$ source counts plus background counts accumulated during $t_{\text {on }} ; N_{\text {off }}=$ background counts accumulated during $t_{\text {off }} ; \alpha=t_{\text {on }} / t_{\text {off }}$ with $t_{\text {off }}=100 \mathrm{~s}$ and $t_{\text {on }}=0.1,1,10$, and $100 \mathrm{~s}$ of burst duration. The $5 \sigma$ thresholds on burst fluence are shown in Table 14.

Line sensitivities.-Five-sigma line sensitivities were estimated using the fractional equivalent width (EW) (Fishman et al. 1982) describing the ratio between line strength (photons $\mathrm{cm}^{-2} \mathrm{~s}^{-1}$ ) and continuum flux (photons $\mathrm{cm}^{-2} \mathrm{~s}^{-1} \mathrm{keV}^{-1}$ ) at energy $E$ within the resolution interval of the line:

$$
\mathrm{EW}=\frac{1.3 k}{E C \varepsilon_{1}}\left[\frac{\left(B+C \varepsilon_{c}\right) W}{A_{\mathrm{geo}} t_{\mathrm{on}}}\right]^{1 / 2},
$$

with $k=$ number of $\sigma ; E=$ photon energy ( $\mathrm{keV}) ; C=$ continuum flux of burst ( photons $\mathrm{cm}^{-2} \mathrm{~s}^{-1} \mathrm{keV}^{-1}$ ), which is a source of background in this case and was taken as the $5 \sigma$ continuum sensitivity value shown in Table 14, multiplied by factors of 1 , 10 , and $100 ; B=$ detector background (counts $\mathrm{cm}^{-2} \mathrm{~s}^{-1}$ $\left.\mathrm{keV}^{-1}\right) ; \varepsilon_{l}$ and $\varepsilon_{c}$ are detection efficiencies for lines and continuum, respectively; $A_{\text {geo }}=$ geometrical detection area $(=615$ $\mathrm{cm}^{2}$ module $\left.{ }^{-1}\right) ; t_{\mathrm{on}}=$ duration of burst observation $(\mathrm{s}) ;$ and $W$ is the FWHM of a line $(\mathrm{keV})$ measured in the pulse-height spectrum. The FWHM is determined by the detector energy resolution, which is assumed to be much larger than the line's natural width.

The results (Table 15) indicate that lines with $\mathrm{EW} \geq 0.3 \%$ will be detected in strong bursts. Previous observations by the KONUS and ISEE 3 (ICE) experiments show that 10\%-20\% of all burst events have spectral line features with typical equivalent widths $\mathrm{EW} \geq 10 \%$ (Hurley 1988).

\subsection{Sensitivity to Polarization Measurements}

The point-source sensitivity for an on-axis celestial source, which is assumed to be $100 \%$ linearly polarized is given by

$$
F_{\min }=\frac{k\left(N_{\mathrm{BGD}}+N_{\text {source }}\right)^{1 / 2}}{\varepsilon A_{\mathrm{eff}} T_{\mathrm{eff}}} .
$$

(See also eq. [7], where $k$ is the statistical confidence level of the detection of COMPTEL and $\varepsilon$ is the detection efficiency for polarization. To good approximation, $\varepsilon=0.5 P_{\max }$, with $P_{\max }$ being the maximal amplitude in Fig. 33.) The factor $\varepsilon$ is determined by the deviation from rotational symmetry of the scattered gamma-ray directions. For the event selections of Figure $33\left(0.75-5 \mathrm{MeV}, 20^{\circ}<\varphi_{\text {geo }}<40^{\circ}\right.$, and $\left.-1.5 \sigma_{\varphi} \leq \bar{\varphi} \leq+1.5 \sigma_{\varphi}\right)$ we obtain $\varepsilon \approx 0.05$. From equation (11) a statistical confidence level of $k=2$ for detecting polarization from a $100 \%$ 
TABLE 14

CONTINUUM SENSITIVITIES $(5 \sigma)$ TO BURST FLUENCE $S\left(\times 10^{-6} \mathrm{ergs} \mathrm{cm}^{-2}\right)$ FOR VARIOUS ENERGY RANGES AND BURST DURATIONS $\left(t_{\mathrm{on}}\right)$

\begin{tabular}{cccccr}
\hline \hline \multirow{2}{*}{$\begin{array}{c}\text { ENERGY } \\
\text { RANGE } \\
(\mathrm{MeV})\end{array}$} & $\begin{array}{c}\langle E\rangle \\
(\mathrm{MeV})\end{array}$ & 0.1 & 1 & 10 & 100 \\
\cline { 3 - 6 } & 0.19 & 0.11 & 0.36 & 1.2 & 5.1 \\
$0.1-0.3 \ldots \ldots$ & 0.55 & 0.16 & 0.50 & 1.7 & 7.1 \\
$0.3-1.0 \ldots \ldots$ & 1.7 & 0.26 & 0.84 & 2.8 & 11.9 \\
$1-3 \ldots \ldots \ldots$ & 5.4 & 0.75 & 2.4 & 7.9 & 34.1 \\
$3-10 \ldots \ldots \ldots$ & & & &
\end{tabular}

polarized Crab-like source $\left(F=3 \times 10^{-3} \mathrm{~cm}^{-2} \mathrm{~s}^{-1}, N_{\mathrm{BGD}}=\right.$ 51,000 counts, $N_{\text {source }}=7000$ counts $)$ is obtained for a 2 week observation period $\left(A_{\text {eff }} T_{\text {eff }}=3 \times 10^{6} \mathrm{~cm}^{2} \mathrm{~s}\right)$. A positive detection of polarization by COMPTEL would require an unusually strong gamma-ray source.

\subsection{Sensitivity to Solar Neutrons}

Data from a "neutron-quiet" period of solar observations were analyzed to estimate the COMPTEL sensitivity to solar neutrons. Gamma-2 events were processed as if neutrons from the Sun were present. The Sun was assumed to be on the telescope axis, and 30 minutes of data were analyzed according to standard selection criteria for solar neutrons.

The lower "proton-equivalent" threshold was taken to be $1.1 \mathrm{MeV}$. Elastic scatter angles were restricted to be below $70^{\circ}$, and event circles were required to come within $10^{\circ}$ of the assumed solar direction (telescope axis). In the energy range $10-150 \mathrm{MeV}, 13$ events passed all criteria and could be taken to be neutrons of solar origin. On the assumption that no neutrons were, in fact, present, this represents a quiescent neutron flux background of $7.2 \times 10^{-4}$ neutrons $\mathrm{cm}^{-2} \mathrm{~s}^{-1}$ for a mean effective neutron detection area of $10 \mathrm{~cm}^{2}$. Thus, the $5 \sigma$ sensitivity (above the quiescent background) is $1.0 \times 10^{-3}$ neutrons $\mathrm{cm}^{-2} \mathrm{~s}^{-1}$. Over a period of $1800 \mathrm{~s}$, this translates to an integrated neutron fluence of 1.8 neutrons $\mathrm{cm}^{-2}$ over the energy range $10-150 \mathrm{MeV}$.

Based on a preliminary COMPTEL analysis of neutron emission from the solar flare on 1991 June 9, additional data restrictions had to be applied in order to reduce the neutron background further. Otherwise, it would have been impossible to distinguish between solar and background neutrons. These additional restrictions (described by Ryan et al. 1992) lead to a further reduction in the effective detection area of neutrons and therefore degraded the solar neutron sensitivity to 5 neutrons $\mathrm{cm}^{-2}$ for a 30 minute observation.

One of the largest neutron flares observed by the GammaRay Spectrometer (GRS) on SMM was that of 1982 June 3 (Chupp et al. 1987). In this event the neutron flux measured above $20 \mathrm{MeV}$ was of the order of $10^{3}$ neutrons $\mathrm{cm}^{-2}$ (Evenson, Meyer, \& Pyle 1983). This flux was inferred through the measurement of neutron decay protons in interplanetary space rather than through direct detection, but it agrees with the extrapolation of the neutron spectrum deduced from the GRS
TABLE 15

Line Sensitivities $(5 \sigma)$ FOR VARIOUS Burst DuRATIONS AND BURST CONTINUUM FLUXES $C^{\mathrm{a}}$

\begin{tabular}{ccccc}
\hline \hline & & & \multicolumn{3}{c}{ EW (\%) } \\
\cline { 3 - 5 } $\begin{array}{c}t_{\text {on }} \\
(\mathrm{s})\end{array}$ & $\begin{array}{c}\text { ENERGY } \\
(\mathrm{MeV})\end{array}$ & $C$ & $10 C$ & $100 C$ \\
\hline $0.1 \ldots \ldots$ & 0.5 & 30.8 & 6.0 & 1.7 \\
$0.1 \ldots \ldots$ & 1.5 & 76.4 & 17.0 & 5.1 \\
$0.1 \ldots \ldots$ & 5.0 & 85.1 & 21.9 & 6.8 \\
$1.0 \ldots \ldots$ & 0.5 & 27.1 & 4.0 & 1.0 \\
$1.0 \ldots \ldots$ & 1.5 & 63.4 & 10.6 & 2.9 \\
$1.0 \ldots \ldots$ & 5.0 & 64.1 & 13.0 & 3.8 \\
$10.0 \ldots \ldots$ & 0.5 & 24.8 & 2.9 & 0.6 \\
$10.0 \ldots \ldots$ & 1.5 & 56.3 & 7.3 & 1.7 \\
$10.0 \ldots \ldots$ & 5.0 & 53.5 & 8.2 & 2.1 \\
$100.0 \ldots \ldots$ & 0.5 & 18.1 & 2.0 & 0.3 \\
$100.0 \ldots \ldots$ & 1.5 & 40.5 & 4.6 & 0.9 \\
$100.0 \ldots \ldots$ & 5.0 & 37.5 & 4.8 & 1.1 \\
\hline
\end{tabular}

${ }^{\mathrm{a}}$ See text.

data. The sensitivity of COMPTEL to neutrons in the energy range $10-150 \mathrm{MeV}$ is thus quite high when compared with earlier observations.

At present, an unknown factor in the neutron sensitivity is the dead time experienced by the telescope in the later phases of the solar flare. Initial neutron observations were affected significantly by the pileup of thermal X-rays persisting for considerably longer periods of time influencing the telescope's ability to register any form of event. A revision of the solar neutron observation mode is underway in order to minimize the thermal X-ray effect. The gamma-ray flux is occasionally intense enough 8 minutes after the onset to affect the subsequent neutron measurements, but this is not normally the case.

\section{OUTLOOK}

After 1 year of operation of COMPTEL in orbit, we can conclude that COMPTEL will make the expected breakthrough in medium-energy gamma-ray astronomy. The COMPTEL sensitivity is more than 10 times higher than that of any previously flown instrument in the $\mathrm{MeV}$ range. COMPTEL, at present, is performing the first complete sky survey at these energies. After its analysis we shall know what the sky looks like at these energies.

From the second year onward, selected objects will be studied in more detail. Part of these observations will be initiated by guest investigators, as NASA has established a very vigorous guest investigator program, which allows scientists outside the instrument team to analyze data or even to suggest special observations.

Prior to the launch of $G R O$, gamma-ray astronomy was largely a field for a restricted group of specialists. With the results of $G R O$ we can expect that gamma-ray astronomy will attract much wider attention and become an integral part of the exciting field of astronomy in general.

\section{REFERENCES}

Bezler, M., Kendziorra, E., Stanbert, R., Hasinger, G., Pietsch, W., Reppin, C., Trümper, T., \& Voges, W. 1984, A\&A, 136, 351

Böhm, G. 1984, Diploma thesis, Technische Univ. München
Brun, R., Bruyant, F., Maire, M., McPherson, A. C., \& Zanarini, P. 1987, GEANT3 User's Guide, Data Handling Division of the Program Library, CERN, Geneva, Switzerland 
Cecil, R. A., Anderson, B. D., \& Madey, R. 1979, Nucl. Instr. Meth., 161, 439

Chupp, E. L., et al. 1987, ApJ, 318, 913

Connors, A., et al. 1991, in Proc. Fourth Int. Workshop on Data Analysis in Astronomy (New York: Plenum), 271

de Boer, H., et al. 1991, in Proc. Fourth Int. Workshop on Data Analysis in Astronomy (New York: Plenum), 241

Diehl, R., \& Graser, U. 1981, Nucl. Instr. Meth., 186, 665

Edwards, A. W. F. 1972, Likelihood (Cambridge: Cambridge Univ. Press)

Evenson, P., Meyer, P., \& Pyle, K. R. 1983, ApJ, 274, 875

Fichtel, C. E., et al. 1983, Proc. 18th Int. Cosmic Ray Conf., 8, 19

Fishman, G., Matteson, J. L., Teegarden, B. J., \& Cline, T. L. 1982, Proposal, BATSE/GRO Spectroscopy Modification (NASA), unpublished

Fishman, G., Meegan, C. A., Parnell, T. A., Wilson, R. B., Paciesas, W., Cline, T., \& Teegarden, B. 1985, Proc. 19th Int. Cosmic Ray Conf., 3, 343

Forster, R. A., Little, R. C., Briesmeister, J. F., \& Hendrichs, J. S. 1990, IEE Trans. Nucl. Sci., 37, 1378

Graser, U., \& Schönfelder, V. 1982, ApJ, 263, 677

Heitler, W. 1954, The Quantum Theory of Radiation (London: Oxford Univ. Press)

Hermsen, W., et al. 1981, Proc. 17th Int. Cosmic Ray Conf., 1, 230

Hermsen, W., et al. 1993, A\&AS, in press

Herzo, D., Koga, R., Millard, W. A., Moon, S., Ryan, J., Wilson, R., Zych, A. D., \& White, R. S. 1975, Nucl. Instr. Meth., 123, 583

Hurley, K. 1988, in AIP Conf. Proc. 170, Nuclear Spectroscopy of Astrophysical Sources, ed. N. Gehrels \& G. Share (New York: AIP), 258

Kippen, M. 1991, Master's thesis, Univ. New Hampshire

Kurfess, J. D., Johnson, W. N., Kinzer, R. L., Share, G. H., Strickman, M. S., Ulmer, M. P., Clayton, D. D., \& Dyer, C. S. 1983, Adv. Space Res., 3 (No. 4), 109

Li, T., \& Ma, Y. 1983, ApJ, 272, 317

Liang, E. P., \& Petrosian, V., eds. 1986, AIP Conf. Proc. 141, Gamma Ray Bursts (New York: AIP), 25

Lockwood, J. A., Hsieh, L., Friling, L., Chen, C., \& Swartz, D. 1979, J. Geophys. Res., 84, 1402
Lockwood, J. A., Webber, W. R., Friling, L. A., Macri, J., \& Hsieh, L. 1981, ApJ, 248, 1194

Mahoney, W. A., Ling, J. C., Jacobson, A. S., \& Tapphorn, R. M. 1980, Nucl. Instr. Meth., 178, 363

Mattox, J. R. 1990, in AIP Conf. Proc. 220, High Energy Gamma Ray Astronomy, ed. James Matthews (New York: AIP)

Matz, S. M., Forrest, D. J., Vestrand, W. T., Chupp, E. L., Share, G. H., \& Rieger, E. 1985, ApJ, 288, L37

Morris, D., et al. 1992, in Proc. Compton Observatory Science Workshop, ed. C. Shrader, N. Gehrels, \& B. Dennis (NASA CP-3137), 102

Pinkau, K. 1966, Z. Nat., A, 21, 2100

Pollock, A. M. T., et al. 1985, A\&A, 146, 352

Pollock, A. M. T., Bignami, G. F., Hermsen, W., Kanbach, G., \& Lichti, G. G. 1981, A\&A, 94, 116

O'Neill, T. J., Ait-Ouamer, F., Morris, J., Tumay Tumer, O., White, R. S., \& Zych, A. D. 1992, in Proc. Compton Observatory Science Workshop ed. C. Shrader, N. Gehrels, \& B. Dennis (NASA CP-3137), 109

Rothschild, M. E., Mushotzky, R. F., Baity, W. A., Gurber, D. E., Matteson, J. L., \& Peterson, L. E. 1983, ApJ, 269, 423

Ryan, J., et al. 1992, in Proc. Compton Observatory Science Workshop, ed. C. Shrader, N. Gehrels, \& B. Dennis (NASA CP-3137), 470

Schönfelder, V., Graser, U., \& Diehl, R. 1982, A\&A, 110, 138

Schönfelder, V., Hirner, A., \& Schneider, K. 1973, Nucl. Instr. Meth., 107, 385

Share, G. H., Matz, S. M., Messina, D. C., Nolan, P. L., Chupp, E. L., Forrest, D. J., \& Cooper, J. F. 1986, Adv. Space Res., 6 (No. 4), 15

Snelling, M., Bennett, K., \& Clear, J. 1986, Nucl. Instr. Meth., A, 248, 545

Strong, A., \& Diehl, R. 1988, in Data Analysis in Astronomy III, ed. V. Di Gesù et al. (New York: Plenum), 55

Strong, A., et al. 1989, in Proc. 21st Cosmic Ray Conf. (Adelaide), 4, 154 Taylor, J. H., \& Weisberg, J. M. 1989, ApJ, 345, 434

von Ballmoos, P., Diehl, R., \& Schönfelder, V. 1987, ApJ, 312, 134

White, R. S. 1968, Bull. Am. Phys. Soc., 13, 714

Winkler, C., et al. 1986, Adv. Space Res., 6 (No. 4), 113 